University of Louisville

ThinkIR: The University of Louisville's Institutional Repository

Electronic Theses and Dissertations

$8-2020$

\title{
Plasmodium impairs antibacterial innate immunity to systemic infections in part through hemozoin-bound bioactive molecules.
}

Christopher Lynn Harding

University of Louisville

Follow this and additional works at: https://ir.library.louisville.edu/etd

Part of the Bacteria Commons, Bacterial Infections and Mycoses Commons, Bacteriology Commons, Biological Phenomena, Cell Phenomena, and Immunity Commons, Immunology of Infectious Disease Commons, Infectious Disease Commons, Medical Immunology Commons, Medical Microbiology Commons, Parasitic Diseases Commons, and the Parasitology Commons

\section{Recommended Citation}

Harding, Christopher Lynn, "Plasmodium impairs antibacterial innate immunity to systemic infections in part through hemozoin-bound bioactive molecules." (2020). Electronic Theses and Dissertations. Paper 3506.

https://doi.org/10.18297/etd/3506

This Doctoral Dissertation is brought to you for free and open access by ThinkIR: The University of Louisville's Institutional Repository. It has been accepted for inclusion in Electronic Theses and Dissertations by an authorized administrator of ThinkIR: The University of Louisville's Institutional Repository. This title appears here courtesy of the author, who has retained all other copyrights. For more information, please contact thinkir@louisville.edu. 


\title{
PLASMODIUM IMPAIRS ANTIBACTERIAL INNATE IMMUNITY TO SYSTEMIC INFECTIONS IN PART THROUGH HEMOZOIN-BOUND BIOACTIVE MOLECULES
}

\author{
By \\ Christopher Lynn Harding \\ B.A. University of Colorado, 2011 \\ M.S. University of Louisville, 2016

\begin{abstract}
A Dissertation
Submitted to the Faculty of the In Partial Fulfillment of the Requirements for the Degree of

Doctor of Philosophy

In Microbiology and Immunology

Department of Microbiology and Immunology

University of Louisville

Louisville Kentucky
\end{abstract} \\ School of Medicine of the University of Louisville
}

August 2020 
Copyright 2020 by Christopher Lynn Harding

All rights reserved 

PLASMODIUM IMPAIRS ANTIBACTERIAL INNATE IMMUNITY TO SYSTEMIC INFECTIONS IN PART THROUGH HEMOZOIN-BOUND BIOACTIVE MOLECULES

\author{
By \\ Christopher Lynn Harding \\ B.A. University of Colorado, 2011 \\ M.S. University of Louisville, 2016 \\ A Dissertation Approved on
}

June 26, 2020

by the following Dissertation Committee

Dissertation Director

Nathan W. Schmidt

Dr. Pascale Alard

Dr. Bing Li

Dr. Matthew B. Lawrenz

Dr. Silvia M. Uriarte 


\section{DEDICATION}

This dissertation is dedicated to my parents

Mr. Martin Lynn Harding

and

Mrs. Susan Ray Harding

For their unwavering love and support in pursuing my interests and goals even though it sent me so far away. Words cannot express my gratitude for your sacrifices, patience, and believing that with hard work and passion I can succeed in anything I do. I am truly privileged to have had these opportunities, and by your example, I will strive to help those who lack this privilege.

"We choose to go to the moon. We choose to go to the moon in this decade and do the other things, not because they are easy, but because they are hard ..."

John F. Kennedy 


\section{ACKNOWLEDGMENTS}

I would like to thank my supervisor, Dr. Nathan W. Schmidt for his support and patience in helping me grow as an independent scientist and for trusting me with a challenging project that became quite different from what he originally intended, in part from the data, but mostly from my stubbornness. Thanks to each of my committee members; Dr. Alard for always being willing to chat about my problems, no matter what they were. Dr. Li, for your enthusiasm about my data at a time when there was so much uncertainty about where my project would go. Dr. Lawrenz, for your help in developing in vitro assays that were new to the lab, for the resources and support in steering the project as far into microbiology as possible, and for being a valuable asset towards advancing my career as a microbiologist. Dr. Uriarte, thank you for your help and resources in pursuing the neutrophil parts of my project and your willingness to chat every time I knocked on your door. A big thank you to Dr. Bob Miller for his help sorting cells but more importantly being a willing and friendly source of useful information at important times.

I also thank current and former members of the Schmidt lab, particularly Dr. Joshua Denny for showing me the ropes in Plasmodium research - and immunology in general- and for your genuine friendliness, sense of humor, and patience the hundreds

times I asked a question out loud and realized the answer before you could respond. I would also like to thank the Microbiology and Immunology Department Admissions 
Committee of 2014 for taking a chance on a prospective student with a sub-mediocre undergraduate GPA, zero publications, decent hands-on experience, and a chip on his shoulder.

Thanks to my many graduate student friends at UofL (especially the wild ones, you know who you are...), our shared trauma brought us close and we will be friends and colleagues forever. Thanks to Dr. Katlin B. Stivers for being a wonderful cohabitator for 5 years at Chez Nous, but more importantly for being a supportive friend, therapist, and flow cytometry guru. I am grateful to my familia Hondureña, both Abners and both Carmens, for taking me as one of their own, supporting my graduate school efforts and for all of the wonderful food, laughs, and music you've shared with me. Thank you to all of my family members back in the Pacific Northwest for listening to me talk about my dissertation project and always supporting my academic pursuits. To Dr. Irina Miralda Molina and Tula Miralda, there is not a better couple of buddies in the world. You have brought me so much happiness that the difficulties of graduate school or life in general could never eclipse it. To my parents, Martin and Sue Harding and the Murphys in Sequim, Amanda, Ryan, Marlee, and Kai, thank you for all your support from afar, for housing and feeding me when I visit and for the texts, phone calls, pictures, artwork, care packages and emotional support while I've been in Louisville. 


\begin{abstract}
PLASMODIUM IMPAIRS ANTIBACTERIAL INNATE IMMUNITY TO SYSTEMIC INFECTIONS IN PART THROUGH HEMOZOIN-BOUND BIOACTIVE MOLECULES

\author{
Christopher L. Harding
}

June 13,2020
\end{abstract}

Despite efforts to decrease the global health burden of malaria, infections with Plasmodium species continue to cause over 200 million episodes of malaria each year which resulted in 405,000 deaths in 2018 [1]. One complication of malaria is increased susceptibility to invasive bacterial infections. Plasmodium infections impair host immunity to non-Typhoid Salmonella (NTS) through activities of heme oxygenase I (HO-I) )-induced release of immature granulocytes and myeloid cell-derived IL-10. Yet, it is not known if these mechanisms are specific to NTS. We show here, that Plasmodium yoelii 17XNL (Py) infected mice had impaired clearance of systemic Listeria monocytogenes (Lm) during both acute parasitemia and up to 2 months after resolution of Py infection that was independent of HO-I and IL-10. Py-infected mice were also susceptible to Streptococcus pneumoniae (Sp) bacteremia, a common malaria-bacteria coinfection, with higher blood and spleen bacterial burdens and decreased survival compared to naïve mice. Mechanistically, impaired immunity to Sp was independent of HO-I, but was dependent on Py-induced IL-10. Splenic phagocytes from Py infected mice exhibit an impaired ability to restrict growth of intracellular Lm, and neutrophils 
from Py-infected mice produce less reactive oxygen species (ROS) in response to Lm or Sp. Analysis also identified a defect in a serum component in Py infected mice that contributes to reduced production of ROS in response to Sp. Finally, treating naïve mice with Plasmodium-derived hemozoin $(\mathrm{Hz})$ containing naturally bound bioactive molecules, excluding DNA, impaired clearance of Lm. Collectively, we have demonstrated that Plasmodium infection impairs host immunity to diverse bacteria, including S. pneumoniae, through multiple effects on innate immunity, and that a parasite-specific factor (Hz+bound bioactive molecules) directly contributes to Plasmodium-induced suppression of antibacterial innate immunity.

IMPORTANCE Our findings provide evidence that there are multiple factors involved in Plasmodium-induced suppression of antibacterial innate immunity. Given the increased mortality rate associated with malaria and invasive bacterial coinfections, these results may provide insight into new approaches to treat those individuals. Finally, this report provides the first description, to our knowledge, of a murine model system of Plasmodium and S. pneumoniae coinfection. In this model, Plasmodium infections impair host immunity to S. pneumoniae, which resulted in increased mortality. As $S$. pneumoniae is one of the most common bacteria identified in individuals with invasive bacterial infections during malaria, this model has the potential to provide further knowledge into the dynamics of this coinfection and identify approaches to treat this coinfection. 


\section{TABLE OF CONTENTS}

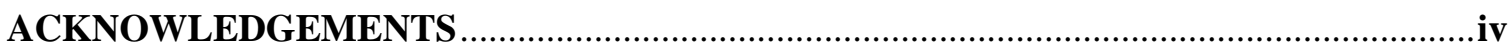

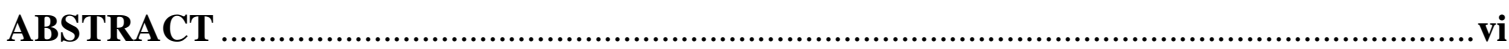

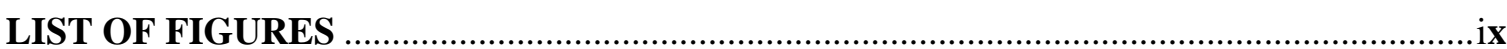

CHAPTER I - GENERAL INTRODUCTION AND METHODS ............................................1

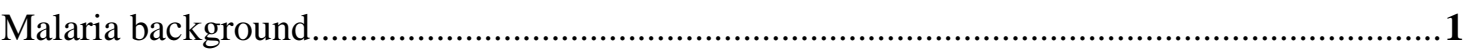

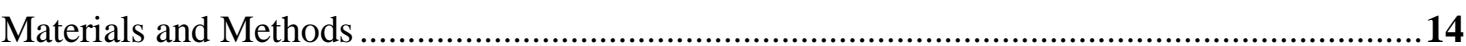

CHAPTER II - CHARACTERIZATION OF MALARIA AND BACTERIAL COINFECTIONS

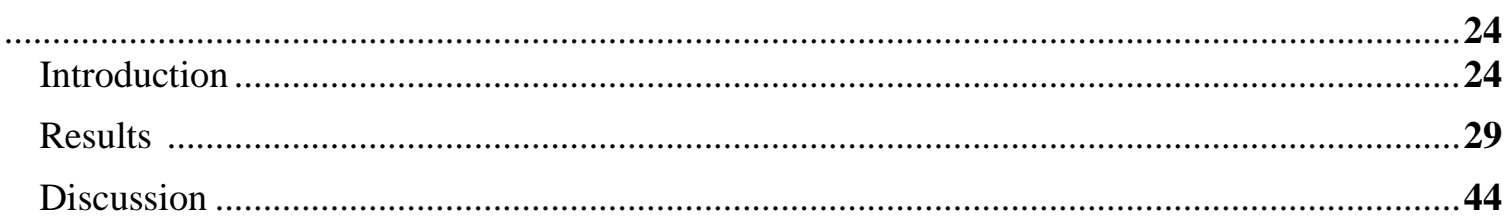

CHAPTER III - IMPACT OF PLASMODIUM YOELII INFECTION ON SPLENIC MYELOID

CELL POPULATIONS AND THEIR ANTI-BACTERIAL EFFECTOR FUNCTIONS.............53

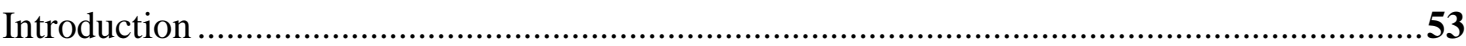

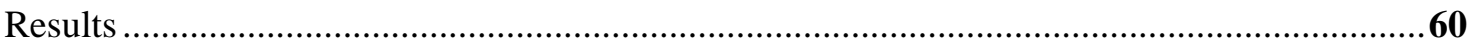

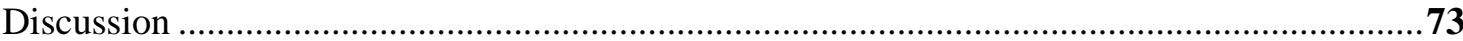

CHAPTER IV - THE PARASITE FACTOR HEMOZOIN IMPAIRS INNATE IMMUNITY TO

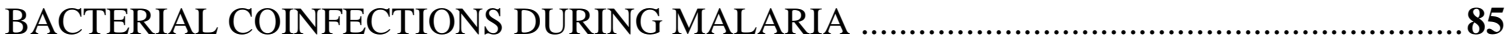

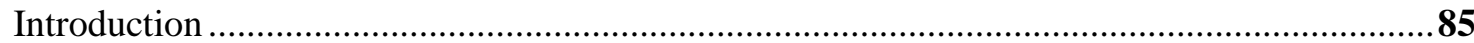

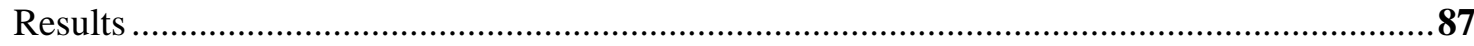

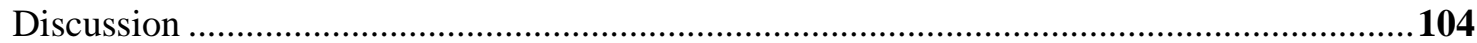

CHAPTER V - FINAL DISCUSSION AND FUTURE DIRECTIONS ................................112

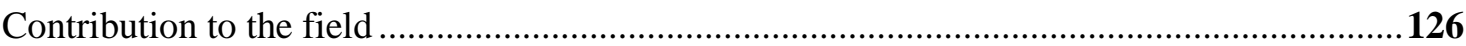

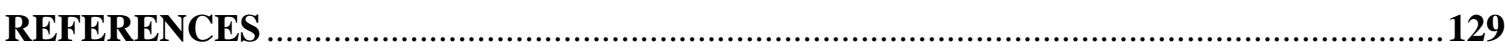

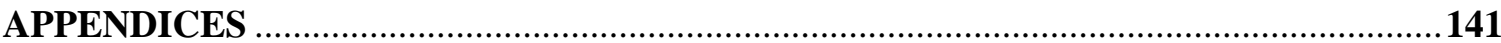

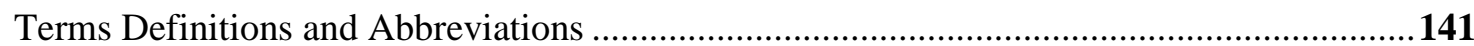

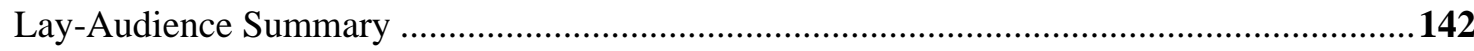

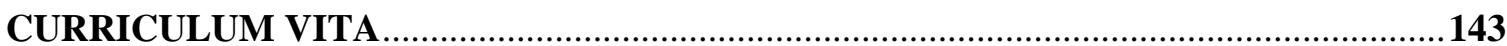




\section{LIST OF FIGURES}

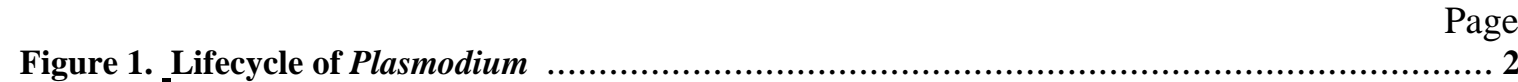

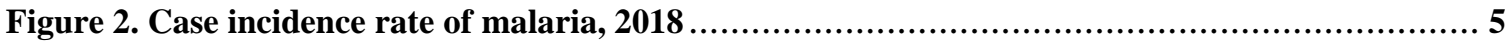

Figure 3 Climate change models predict increase in vector stability in Europe .......................... 6

Figure 4. $P$. yoelii infection induces long-term suppression of anti-bacterial innate immunity during bacteremia. 30

Figure 5. IFN $\gamma$ production during early and late coinfection Py infection confers protection against wildtype Lm. .34

Figure 6. Py infection confers protection against wildtype Lm. 36

Figure 7. Py induction of HO-1 and IL-10 impair immunity to NTS but not Lm....................... 38

Figure 8. P. yoelii infection increases severity of Pneumococcal infection. .............................. 40

Figure 9. Py infection induces long-term impaired immunity to Sp infection. ....................... 41

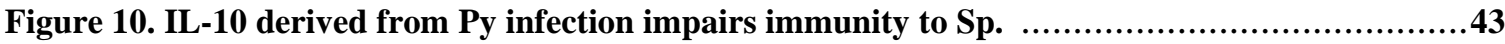

Figure 11. Gating strategy for splenic myeloid cell populations ............................................62

Figure 12. P. yoelii-induced temporal changes in splenic myeloid cell populations do not correlate with impaired immunity during bacteremia.

Figure 13. Analysis of Eosinophils and CD11 ${ }^{\text {hi }}$ Dendritic Cell populations over the course of $P$. yoelii infection. .65

Figure 14. Splenic PMNs from $P$. yoelii infected mice have defective ROS production in response to Lm. 68

Figure 15. Splenic PMNs from $P$. yoelii infected mice have defective ROS production in response to heat-killed Sp.

Figure 16. P. yoelii infection impairs anti-bacterial functions of splenic phagocytes.................... 72

Figure 17. Hemozoin isolation from liver and spleens of Py-infected mice. 88

Figure 18. PyHz treatment does not alter intracellular ROS production of splenic neutrophils in whole spleen single cell suspension. 
Figure 19. Hemozoin treated bone-marrow-purified Neutrophils do not have defective ROS responses.

Figure 20. Infection with Plasmodium berghei mutant deficient in $\mathrm{Hz}$ does not delay $\mathrm{Lm}$ clearance.

Figure 21. P. yoelii -derived hemozoin and bound bioactive molecules impair anti-bacterial functions of splenic phagocytes. 97

Figure 22. P. yoelii hemozoin with bound bioactive molecules is sufficient to impair clearance of $\mathrm{Lm}$ in vivo. 98

Figure 23. Plasmodium falciparum hemozoin $(\mathrm{PfHz})$ is sufficient to impair clearance of $\mathbf{L m}$ in vivo.

Figure 24. Hemozoin preperation differentially impacts anti-bacterial innate immunity. 102

Figure 25. P. yoelii hemozoin with bound bioactive molecules does impair immunity to Sp. 104

Figure 26. Complement depletion during malaria. 121

Figure 27. Potential mechanisms of Plasmodium-driven immune suppression to bacterial coinfections. 127

Figure 28. Dissertation Summary Schematic: Mechanisms of Plasmodium infection-driven suppression of antibacterial innate immunity to invasive coinfections 128 


\section{CHAPTER I}

\section{GENERAL INTRODUCTION AND METHODS}

\section{Malaria background}

Despite the advances in vaccine development and treatments of infectious diseases in recent years, protozoan parasites of the Plasmodium genus cause over 200 million cases of malaria each year and in 2018 resulted in an estimated 405,000 deaths. The overwhelming majority of these fatal malaria cases occur in Africa (94\% of total fatalities 2018) and children under 5 years old are most susceptible (67\% of deaths worldwide in 2018) [1, 2]. Malaria is an ancient and widespread disease, caused by infection with protozoan parasites of the Plasmodium genus. As one of the most successful pathogens, Plasmodium has plagued humans for the entirety of our history, with the disease being mentioned in historical writings of ancient societies in China, India, Mesopotamia, Egypt and Greece [3]. Recent discoveries suggest that malaria significantly pre-dates human history, as dinosaurs were almost certainly infected with malaria-like parasites of the Paleohaemoproteus species. Supporting the theory that malaria may have played a role in extinction of the dinosaurs, 100 million year-old fossils of biting midge insect vectors have been found from the early Cretaceous period containing P. burmacis parasites [4]. Almost 200 species of Plasmodium have been described to date, infecting a wide variety of vertebrate hosts, including birds, reptiles, and other mammals [5]. There are currently around 30 different species of Plasmodium that are known to naturally infect non-human primates, and 5 are known to infect 
humans, the most deadly of which is Plasmodium falciparum [6,7]. Plasmodium

employs female Anopheles mosquitoes as primary hosts and vectors that transmit the

parasite to vertebrate secondary hosts while the mosquito acquires a bloodmeal [8].

As its name suggests, malarial disease was historically attributed to "bad air" due

to the early association between the disease and proximity to swamps and marshlands. In

1897, Ronald Ross was able to demonstrate presence of pigmented parasite structures

within mosquitos that had fed on blood of malaria patients, which then invaded the

mosquitos' salivary glands [3]. The parasite stage found in mosquito salivary glands is

known as sporozoites, which are infectious to vertebrate hosts when transmitted by the

bite of an infected mosquito (Figure 1).

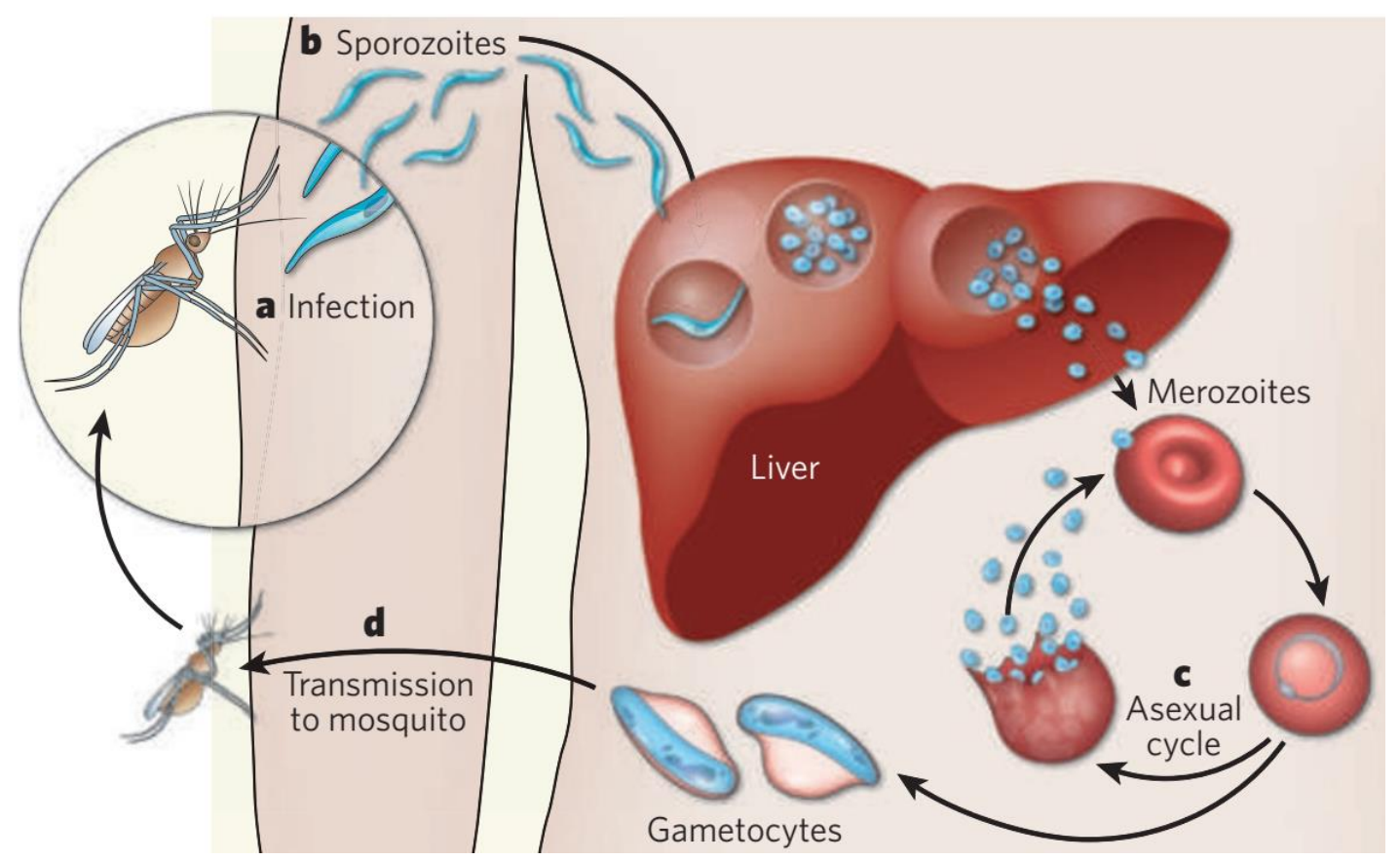

Figure 1. Lifecycle of Plasmodium Adapted from Michalakis and Renaud Nature 2009 "Malaria:

Evolution in vector control" [9]. a. Female Anopheles mosquitoes requires a blood meal for development of their eggs. In the process, infected females inject the sporozoite form of the parasite into a human host. b. Sporozoites are carried in the bloodstream to liver cells, where they proliferate asexually, and then, as merozoites, invade red blood cells. c. Replication within red blood cells initiates the blood stage of malaria, which manifests as fever and chills. This precedes the production of male and female gametocytes that are transmitted back to a mosquito during a blood meal, where they fuse to form oocysts that divide to create sporozoites. These migrate to the mosquito salivary glands, where the cycle of infection starts again. Details of the life cycle differ for different species of Plasmodium. Development within the mosquito takes 10-14 days or longer. 


\section{Parasite lifecycle and malaria disease}

Within the human host, a small proportion of sporozoites exit the skin dermal layer by migrating to and penetrating a blood vessel within 1-3 hours [10]. From there, sporozoites travel through the bloodstream to the liver and actively transverse a capillary barrier, called sinusoids, until suitable hepatocytes (liver cells) are invaded. Here they undergo a process called schizogony, asexual reproduction where individual sporozoites divide and differentiate into merozoite daughter cells [11]. The liver-stage of Plasmodium's lifecycle is clinically asymptomatic, making it an ideal target for vaccine development. Despite significant efforts, to date there are still no highly effective vaccines that target the liver stage [12]. Parasites within hepatocytes undergo schizogony within a membrane-bound vesicle, known as merosomes, which develop for 10-12 days in the liver during $P$. falciparum infection. Merosomes are released from hepatocytes and enter the blood where they rupture and give rise to up 40,000 merozoites per merosome. In the bloodstream merozoites go on to infect erythrocytes (red blood cells), initiating the blood-stage of the Plasmodium lifecycle [13].

Blood-stage malaria consists of a chronic cycle of asexual schizogony where merozoites infect a new erythrocyte, undergo multiple rounds of intracellular asexual reproduction for a period of 48 hours. Infected erythrocytes exhibit synchronized development and their simultaneous release from host cells and elicit a robust inflammatory immune response [14]. This 48-hour lifecycle, combined with an apparent ability to sense the hosts' circadian rhythm, is thought to either increase chances for transmission to a new mosquito vector $[15,16]$, or for optimal avoidance of host immune responses when extracellular parasites are most vulnerable $[17,18]$. This timed parasite 
release into the bloodstream is also responsible for the characteristic cycling fevers generally associated with malaria.

Symptoms of uncomplicated malaria include bouts of fevers lasting 6 to 10 hours, headaches, nausea and vomiting, as well as body aches and general malaise.

Manifestations of severe malaria are more likely to occur in children under 5 years old, pregnant women, immune-compromised individuals such as those with HIV/AIDS, and non-immune persons or travelers who have not had previous exposure to Plasmodium [19]. The complications of severe malaria are often fatal and can materialize as cerebral malaria, severe anemia, acute respiratory distress syndrome, and others related to hyperparasitemia, which refers to a high proportion of infected red blood cells (greater than 5\% in humans) [20]. Enlargement and swelling of the liver and spleen are also common and splenic rupture is possible, though quite rare. Since symptoms of malaria do not differ significantly from many other infectious diseases, diagnosis of malaria generally requires the demonstration of parasites in a blood smear [21]. Plasmodium infections that are left untreated can last from several weeks to months, and prolonged low-level (low parasitemia), asymptomatic infections can last several years with delays in clinical symptoms as high as 2, 4, or even 8 years after exposure [22-24].

\section{Malaria geography and burdens of disease}

The geographical range of malarial disease is currently restricted to tropic and subtropical regions (Figure 2). Global climate change threatens to increase vector stability in regions that have had little to no transmission of malaria in the past century, as the map below indicates for Europe (Figure 3). 


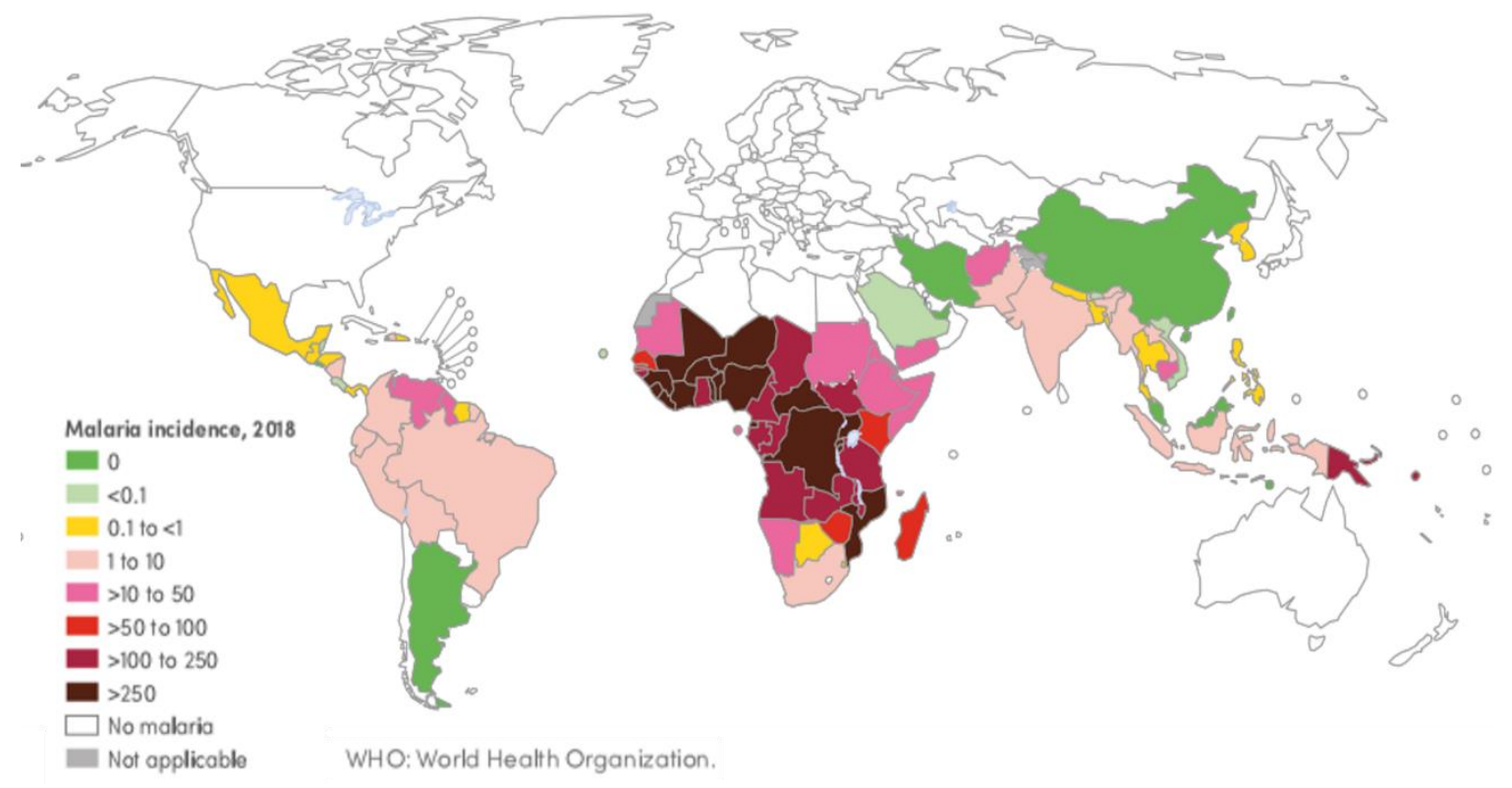

Figure 2. Case incidence rate of malaria, 2018 (cases per 1,000 population at risk). Adapted from World Malaria Report 2019

Among the many reasons that the massive efforts to "eradicate" malaria have fallen short so far, the prevalence and persistence of the Anopheles mosquito vector, maintenance of the parasite within its human reservoir (including asymptomatic infections), and the emergence of drug and insecticide resistance represent major challenges to Plasmodium eradication [25-29]. Malaria disproportionally impacts parts of the world with the highest levels of poverty, which is clear when considering countries that eradicated malaria in the second half of the $20^{\text {th }}$ century had significantly higher economic growth after eliminating the disease than their malaria plagued neighbors [30]. The debilitating effects of moderate and even mild malaria cases also have a major negative impact on cognitive function, learning, and the number economic opportunities individuals in malaria endemic regions are able to capitalize from [31, 32]. When considering the economic burdens and time spent caring for infected individuals, the consequences of malaria transmission are even greater. 

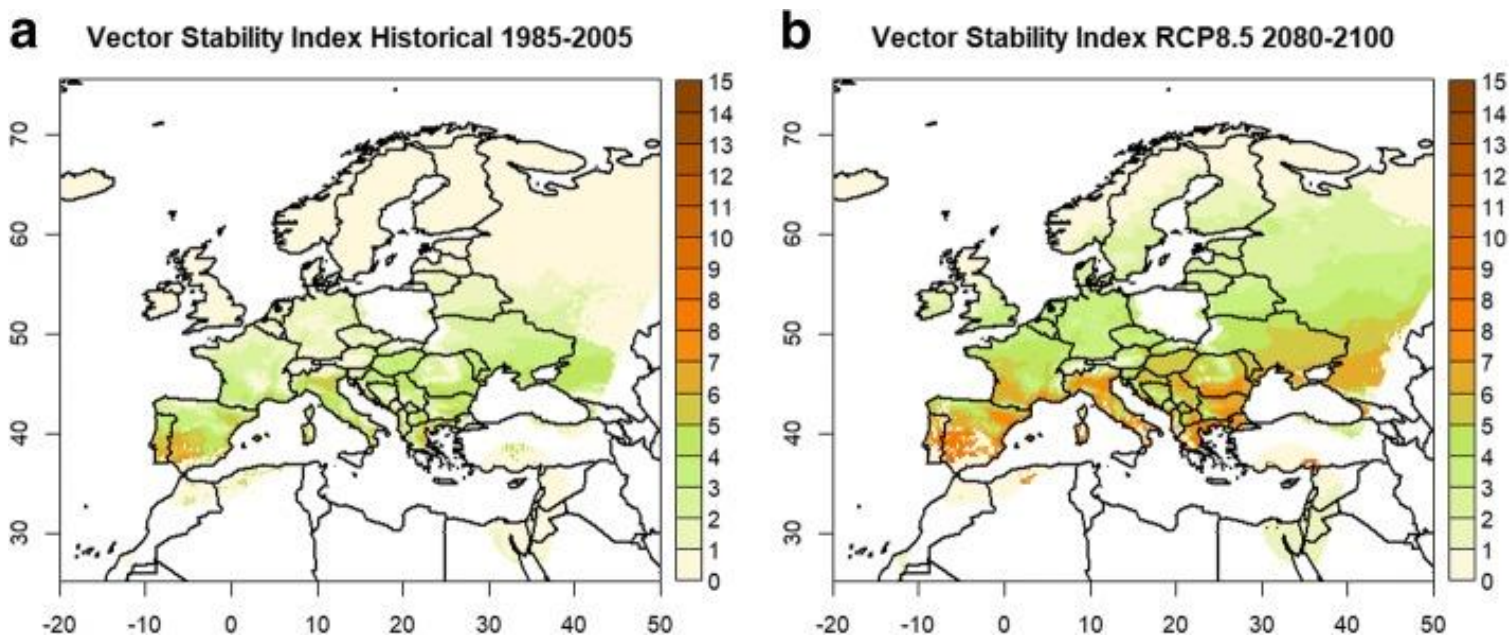

Figure 3 Climate change models predict increase in vector stability in Europe. Adapted from Hertig 2019 (Parasit Vectors) [33].

\section{Immune response during malaria}

The clinical disease 'malaria' is mainly driven by the host's inflammatory immune response, where a carefully regulated effort is needed to be able to successfully clear the parasite while also avoiding collateral damage of host tissues. Although the host immune system recognizes infected hepatocytes, failure to eliminate every infected hepatocyte results in progression to the blood-stage of infection [34]. At the onset of parasite replication in the bloodstream, cells of the innate immune system recognize molecular signatures of the parasite that activate them to either engulf and kill cell free parasites and infected red blood cells (a process called phagocytosis), produce toxic substances that kill Plasmodium, and attract other immune cells that function to eliminate Plasmodium [35]. Innate cells such as dendritic cells and macrophages can act as antigen presenting cells that communicate with the adaptive immune system by presenting pieces of the parasite directly or produce chemical signals that help initiate an adaptive immune response that will be developed specifically to the invading microorganism. Neutrophils, also called polymorphonuclear neutrophils (PMN), respond to Plasmodium infection 
through the release of reactive oxygen species (ROS) and granules, which are internal compartments that store other toxic molecules capable of killing Plasmodium parasites and other microorganisms [36]. While PMNs are quickly mobilized, they generally live for 24 hours or less. In contrast, macrophages are long-lived cells capable of phagocytosing and killing parasites and infected red blood cells [37]. Macrophages in the spleen, an organ that functions to filter the blood, normally uptake and remove damaged red blood cells. During Plasmodium infection macrophages phagocytose free parasites and infected red blood cells, thereby preventing uncontrolled replication that would lead to hyperparasitemia [38, 39].

Another aspect of the immune system that is involved in infections is the complement system. Components of this system can bind pathogens directly causing their destruction and send recruitment signals to attract immune cells. Infected cells or pathogens bound with complement components are "tagged" for destruction by innate immune cells. Complement also interacts and cooperate with antibodies as a separate mechanism for improved pathogen neutralization [40]. While the innate immune system protects the host from an overwhelming Plasmodium infection, efficient clearance of Plasmodium is mediated by the ensuing adaptive immune system [41].

\section{Malaria and secondary coinfections}

In Plasmodium endemic areas, malaria was identified as a major risk factor for developing bacteremia that correlated with a worse clinical outcome and higher morbidity [42-49]. Evidence of bacterial coinfections with malaria have been found in analysis of 4 heads of mummies from ancient Lower Egypt in ca. 800, where DNA analysis found the co-occurrence of $P$. falciparum with Tuberculosis [50]. More 
recently, in four separate clinical studies conducted in sub-Saharan Africa, between 4 and $23 \%$ of children with severe malaria had concomitant sepsis $[42,49,51,52]$. A population-based case study found that in a malaria-endemic region of Kenya, authors estimated that malarial disease predisposed children to bacteremia and accounted for more than half of all bacteremia cases [42]. Finally, a systematic review of clinical studies revealed that African children (from 25 different studies in 11 different countries that met criteria) with recent or acute malaria are at risk of bacterial infection and that such coinfections resulted in increased risk of mortality [53]. Gastrointestinal and pulmonary bacterial pathogens such as non-typhoid Salmonella (NTS) and Streptococcus pneumoniae (Sp), respectively, are the most common organisms isolated from blood of Plasmodium-bacteria co-infected individuals [42, 45, 49, 51, 52, 54, 55].

A 2010 meta-review of invasive bacterial infections in Africa reported that across all studies having appropriate diagnostic methods, approximately $6.5 \%$ of patients with Plasmodium positive blood smears also had a concomitant bloodstream fungal or bacterial infection [45]. Several clinical reports have identified NTS as the most prevalent bacterial pathogen isolated in blood of malaria patients and some also mention that this trend is especially prominent during rainy seasons, when transmission of Plasmodium falciparum is more frequent [56]. Clinicians also observed a difference in the pathogenic nature of NTS infections occurring simultaneously with malaria. Normally, NTS infections are limited to the gastrointestinal tract and result in overt diarrheal disease with little to no systemic invasion to other tissues due to control of infection via the innate immune response [57]. However, during coinfection with Plasmodium, NTS is able to colonize the intestines and disseminate systemically, 
resulting in bacteremia [58]. The recognition of strong correlation between malaria and invasive bacterial infections from analysis of clinical observations has provided researchers with sufficient rationale to investigate the mechanisms by which Plasmodium infection increases host susceptibility to NTS. Currently, studies conducted with both mouse models of malaria and samples from malaria patients suggest that enhanced susceptibility to NTS is due to hemolysis and anemia related to the parasite infection, but have not identified a parasite factor to be involved [59-64].

\section{Other malaria coinfections:}

Other than NTS and Sp coinfections with malaria, the incidence of malaria with several other concomitant bacterial, viral, fungal, and protozoan pathogens have been reported. A long history of malaria occurring simultaneously with Tuberculosis is indicated by researchers who have found evidence of this coinfection during molecular analysis of Egyptian mummy remains [50]. Although incidence of malaria is geographically associated with rural areas and tuberculosis is more prevalent in areas with higher population density, there are reports of malaria occurring in $3 \%$ of tuberculosis patients in an epidemiological study in Tanzania and another in Angola demonstrated malaria coinfections in over $37 \%$ of tuberculosis patients $[65,66]$. Finally, a study in Guinea-Bissau found that malaria was associated in an increased mortality in tuberculosis infected individuals and showed an improved outcome for tuberculosis patients when transmission of malaria was prevented [48]. Given the general endemicity of malaria and tuberculosis, studies have been conducted to determine the interaction between the Mycobacterium bovis (BCG) vaccine strain, or the fully virulent 
Mycobacterium tuberculosis, which causes human tuberculosis disease, and immunity to

malaria. While some studies demonstrated that BCG conferred protection against a

normally lethal Plasmodium strain in a murine model, others have shown either enhanced

severity of malaria or exacerbation of acute BCG infection or M. tuberculosis (see Table

1) $[67]$.

Table 1. Malaria-Tuberculosis coinfection studies in murine models. These studies suggest tuberculosis infection can modulate immune response to subsequent Plasmodium infection and influence the clinical outcome. Adapted from dissertation of R. Frita, 2014 University of Lisbon, "Malaria and Tuberculosis coinfection: a role for Hemozoin immunosuppression"

\begin{tabular}{|c|c|c|c|c|}
\hline Mycobacterium sp & Plasmodium $\mathrm{sp}$ & $\begin{array}{l}\text { Mice } \\
\text { strain }\end{array}$ & Findings/Outcome & Reference \\
\hline $\begin{array}{l}\text { M. bovis BCG } \\
\text { (Tokyo) }\end{array}$ & P. yoelii $17 \mathrm{XL}$ & $\mathrm{A} / \mathrm{J}$ & $\begin{array}{l}\text { BCG protects mice from lethal } \\
\text { infection with } P \text {. yoelii. Protection } \\
\text { was associated with higher levels of } \\
\text { Th1 cytokines. }\end{array}$ & $\begin{array}{l}\text { Matsumoto S. } \\
\text { (Yamada T.), } \\
2001^{[170]}\end{array}$ \\
\hline $\begin{array}{l}\text { M. tuberculosis } \\
\text { (CDC1551) }\end{array}$ & P. yoelii 17X NL & C57BL/6 & $\begin{array}{l}22 \% \text { co-infected mice died vs } 10 \% \\
\text { in } P \text {. yoelii group. Lethality was } \\
\text { associated with higher bacterial } \\
\text { burdens and higher levels of IFN- } \gamma \\
\text { in co-infected animals }\end{array}$ & $\begin{array}{l}\text { Scott C. } \\
\text { (Manabe Y.), } \\
2004^{[171]}\end{array}$ \\
\hline $\begin{array}{l}\text { M. tuberculosis } \\
\text { (CDC1551) }\end{array}$ & P. yoelii $17 \mathrm{XL}$ & $\begin{array}{l}\text { C57BL/6 } \\
\text { BalbC }\end{array}$ & $\begin{array}{l}\text { Exposure to } M \text {. tuberculosis } \\
\text { conferred protection to C57BL/6 } \\
\text { but not BalbC mice through } \\
\text { enhancement of Th-1 response }\end{array}$ & $\begin{array}{l}\text { Page K, } \\
\text { (Manabe Y.), } \\
2005^{[172]}\end{array}$ \\
\hline $\begin{array}{l}\text { M. bovis BCG, } \\
\text { (Copenhagen) }\end{array}$ & P. chabaudi AS & $\begin{array}{l}\text { C57BL/6 } \\
\text { A/J }\end{array}$ & $\begin{array}{l}P \text {. chabaudi infection in previously } \\
\text { BCG infected animals became } \\
\text { lethal in C57BL } 6 \text { mice whilst it } \\
\text { conferred protection to } \mathrm{A} / \mathrm{J} \text { mice. } \\
\text { Lethality was associated with an } \\
\text { earlier and stronger Th- } 1 \text { type } \\
\text { response in } \mathrm{C} 57 \mathrm{BL} / 6 \text { mice than } \mathrm{A} / \mathrm{J}\end{array}$ & $\begin{array}{l}\text { Leisewitz A., } \\
2008^{[173]}\end{array}$ \\
\hline $\begin{array}{l}\text { M. bovis BCG } \\
\text { (Pasteur) }\end{array}$ & P. chabaudi AS & C57BL/6 & $\begin{array}{l}\text { Infection of C57BL } / 6 \text { mice with } P \text {. } \\
\text { chabaudi exacerbated acute BCG } \\
\text { infection and promoted reactivation } \\
\text { of latent mycobacterial infection }\end{array}$ & $\begin{array}{l}\text { Hawkes M. } \\
\text { (Kain K.), 2010[174] }\end{array}$ \\
\hline $\begin{array}{l}\text { M. tuberculosis } \\
\text { (H37Rv) }\end{array}$ & P. berghei NK65 & $\mathrm{C} 57 \mathrm{BL} / 6$ & $\begin{array}{l}\text { Co-infection exacerbated chronic } \\
\text { tuberculosis while protecting mice } \\
\text { against Plasmodium infection. } \\
\text { Exacerbation of tuberculosis was } \\
\text { associated with uncontrolled } \\
\text { production of inflammatory } \\
\text { cytokines by innate cells. }\end{array}$ & $\begin{array}{l}\text { Mueller A-K } \\
\text { (Schneider B.), } \\
2012^{[175]}\end{array}$ \\
\hline
\end{tabular}

Other respiratory bacterial infections such as Haemophilus influenzae,

Staphylococcus aureus, and Pseudomonas aeruginosa or gastroenteric pathogens like E. 
coli and cholera have been identified in patients with malaria, but these coinfections are not as commonly detected as NTS, Sp or tuberculosis - mentioned above.

\section{Malaria HIV coinfections}

With the multitude of information known about immune suppression and increased susceptibility to secondary infections in HIV positive individuals, and the large overlap in geographical endemicity of HIV and malaria, it is crucial to understand the interactions between the two pathogens and how they may influence an individual's immune response to an invasive bacterial infection. It has been demonstrated that malaria can enhance viral replication in HIV coinfections and reactivate latent infection [68-70]. Malaria-driven cytokine responses and inflammation contribute to this by inducing the activation of $\mathrm{CD} 4^{+} \mathrm{T}$ cells and rendering them more susceptible to viral invasion and replication [69]. This coinfection was associated with higher viral loads in a clinical study in rural Malawi and East sub-Saharan Africa [69, 71]. It has also been shown in vitro that treatment with the parasite by-product, hemozoin, increases the HIV RNA load in human peripheral blood mononuclear cells (PBMCs), as well as lead to functional defects in monocyte-derived DCs and monocytes which impede maturation and promote transfer of HIV-1 to CD4+ T-cells [72-74].

In respect to the work exhibited here, due to the widespread incidence of HIV in sub-Saharan Africa, it will be important for future studies to examine how HIV and AIDS complicates and likely further impairs immunity to bacterial coinfections during malaria. It would also be diligent of clinicians to make an intentional effort to test for and report HIV status, tuberculosis infection, and any acute or recent malaria episodes when 
accumulating data or presenting clinical reports. This information is important to provide researchers with a more complete picture of the interplay between these three highly endemic pathogens so that we may design more appropriate studies to explore their complex interactions and mechanisms of manipulating host immunity to invasive bacterial diseases.

The risk of developing bacterial sepsis is significantly higher in individuals with severe malaria and this combination is associated with increased mortality $[49,75,76]$. This major global health problem is compounded by the lack of availability of accurate testing in malaria endemic areas. Additionally, while malaria is commonly over diagnosed, only one third of cases of invasive bacterial disease are accurately diagnosed, leading to the misuse of antibiotic treatments [75]. Aside from the difficulties of obtaining the information in the clinic for optimal treatment of patients with these coinfections, there is a fundamental lack of understanding of the mechanisms that increase host susceptibility to invasive bacterial disease during malaria. The aim of this work is to expand our knowledge of the factors by which the Plasmodium parasites impair host immunity to bacterial coinfections, and expand beyond what has already been learned about coinfections with non-Typhoid Salmonella. In this work I also seek to provide evidence that the parasite factor, hemozoin, contributes to impaired bacterial immunity, introduce a novel method of hemozoin isolation and the first murine model of Plasmodium Pneumococcal coinfection. Scientific efforts in all fields related to malaria are of critical importance considering the inextricable connection between this disease and poverty human suffering. A temporally suitable comparison can be made between the current pandemic with COVID-19 which has had major global consequences. At the time 
this is written, there are nearly 7.5 million confirmed cases and 420,000 deaths from COVID-19 worldwide, while in 2018 malaria infected 200 million people and killed over 400,000 people $[2,77]$. Although the mortality rate of COV-SARS2 is considerably higher, this comparison offers a useful way to reflect on the overall public health burden of malaria. For these reasons, it is essential that we improve the public health of the impoverished communities most heavily affected by conducting research that aims to understand the interactions between Plasmodium and host immunity, as well as the other comorbidities and factors that influence malaria-related morbidity. 


\section{Materials and Methods}

\section{Mice}

Female C57BL/6N mice 6-12 weeks old were obtained from Charles River Laboratories (Wilmington, MA) and housed at the University of Louisville under appropriate biosafety level conditions. All mice were housed in a specific pathogen-free facility and acclimatized for a minimum of 7 days before starting experiments. Male mice are generally more prone to higher parasitemia, more severe Plasmodium infections, and often become moribund [78-80]. Since we are not interested in gender differences, and to have more comparable groups, only female mice will be used for all experiments Animals were fed the NIH-31 diet (Modified Open Formula Mouse/Rat Irradiated Diet; Harlan 7913; Envigo, Indianapolis, IN) and provided autoclaved, non-acidified reverseosmosis water ad libitum. The mice were kept on a 12-hour light/dark cycle from 6 AM to $6 \mathrm{PM}$ and 6PM to 6AM, respectively. All animal handling and experimentation were reviewed and approved by the University of Louisville Institutional Animal Care and Use Committee based on the recommendations of the Guide for the Care and Use of Laboratory Animals of the National Institutes of Health.

\section{Infections}

Plasmodium yoelii: Mice were infected i.v. with $5 \times 10^{6}$ parasitized red blood cells (pRBC) Plasmodium yoelii 17XNL (Py). Parasitemia (\% of infected red blood cells) was determined by analyzing samples of peripheral blood obtained from day 5 post Py infection and alternating days afterward until mice cleared the infection. Blood samples were fixed in $0.02 \%$ glutaraldehyde and stained with antibodies specific to CD45-APC 
(clone 104) and Ly-76 (clone Ter119) from Biolegend (San Diego, CA) and dyes dihydroethidium, and Hoechst 33342 (Sigma-Aldrich, St. Louis, MO). Parasitemia was analyzed with flow cytometry using a BD Fortessa, where pRBCs were identified as the population of $\mathrm{CD}^{4} 5^{-}$, Ter $119^{+}$, dihydroethidium ${ }^{+}, \mathrm{Hoechst}^{+}$cells. All flow cytometry data was analyzed with FlowJo software.

Plasmodium berghei: Mice were infected via intraperitoneal injection with $1 \times 10^{4}$ pRBCs of strain NK65 or mutants obtained from peripheral blood preparations of infected donor mice. In the NK65 infections were initiated with wild type, $\Delta d p a p, \Delta p m 4$ strains kindly provided by Dr. Phileppe Van den Steen at University of Leuven, Belgium [81]. Parasitemia was tracked on days 6, 7, and 9 post infection and Lm infection was initiated as with Py coinfections, on day 7 post $\mathrm{Pb}$. Parasite burdens were determined as described for Py.

Listeria monocytogenes: Strain 1043S actA-deficient Listeria monocytogenes, or 1043S wild type, were grown from freezer stock aliquot in tryptic soy broth and then washed with PBS and resuspended in sterile saline. Mice were infected i.v. with $5 \times 10^{6}\left(\mathrm{ActA}^{-}\right)$, or $1 \times 10^{4}($ wildtype $1043 \mathrm{~S}) \mathrm{CFU}$ on indicated days post Py infection or as an initial infection in age-matched mice.

Streptococcus pneumoniae: S. pneumoniae (Sp) ATCC strain 6303, a type 3 capsule isolate cultures were started from freezer stocks and grown in brain heart infusion broth containing $50 \mathrm{mM}$ HEPES buffer and incubated statically at $37^{\circ} \mathrm{C}$ with $5 \% \mathrm{CO}_{2}$. Cultures were washed in PBS and resuspended in sterile saline. Mice were infected with $1 \times 10^{6} \mathrm{CFU}$ of Sp intranasally to naïve mice or Py infected mice on day 7 post Py 
infection in a volume of $30 \mu 1$. Inoculum dose was confirmed by plating serial dilutions on blood agar and counting colonies after 12 to 18 hours of growth at $37^{\circ} \mathrm{C}$ with $5 \% \mathrm{CO}_{2}$. Salmonella enterica serovar Typhimurium (NTS): Frozen aliquots of Salmonella enterica serovar Typhimurium strain 12023 were thawed and resuspended in LB broth and grown shaking at $37^{\circ} \mathrm{C}$. Cultures were then washed in sterile $1 \mathrm{X}$ PBS and resuspended in sterile saline. Mice were infected on day 10 post Py infection via intraperitoneal injection of $1 \times 10^{3} \mathrm{cfu}$ in $200 \mu \mathrm{l}$. Inoculum dose was verified by plating serial dilutions on LB agar containing $50 \mu \mathrm{g} / \mathrm{mL}$ carbenicillin.

\section{Bacterial burden quantification analysis}

Spleens and livers from $\mathrm{Lm}$ infected mice were collected in $0.2 \%$ IGEPAL (Sigma Aldrich, St. Louis, MO) at the indicated time points. Organs were homogenized and plated in serial dilutions on tryptic soy agar plates with $50 \mu \mathrm{g} / \mathrm{mL}$ streptomycin. Lungs and spleens from Sp infected mice were collected in sterile 1X PBS. Organs were homogenized and plated in serial dilutions on tryptic soy agar plates supplemented with $10 \%$ sheep blood (company, city state). Peripheral blood was collected via retro-orbital bleed and plated directly on tryptic soy agar plates with $10 \%$ sheep blood agar. For analysis of bacterial burdens in NTS infections, peripheral blood and organ homogenates were plated in serial dilutions on LB agar plates containing $50 \mu \mathrm{g} / \mathrm{mL}$ carbenicillin. 


\section{In vivo Inhibition of IL-10 and HO-1}

Py-infected and naïve mice were injected i.p. with 300, 200, and $100 \mu \mathrm{g}$ of rat anti-IL-10 IgG1 kapa monoclonal antibody (clone JES5-2A5) or rat anti-horseradish peroxidase $\mathrm{IgG1}$ isotype control (InVivoMAb, West Lebanon, $\mathrm{NH}$ ) in $0.2 \mathrm{~mL}$ sterile saline on days Py+6, 7, and 8 for $\mathrm{Lm}$ coinfection (day Lm -1, 0, and +1 ), days Py+7, 8 , and 9 for Sp coinfection (day Sp -1, 0, and +1), and days Py+9, 10, and 11 (days NTS -1, $0,+1)$ for NTS coinfections. Similarly, Py-infected and naïve mice were treated i.p. with $40 \mu \mathrm{mol} / \mathrm{kg}$ Tin Protoporphyrin IX (chloride) (Sn PP) (Cayman Chemical, Ann Arbor, MI) in $0.2 \mathrm{~mL} 1 \mathrm{X}$ PBS on days Py+5, 6, 7, and 8 for $\mathrm{Lm}$ coinfection (day Lm -2, $-1,0$, and +1), days Py+6, 7, 8, and 9 for Sp coinfection (day Sp -2, -1, 0, and +1), and days Py $+8,+9,10$, and 11 (days NTS $-2,-1,0,+1$ ) for NTS coinfections.

\section{In vivo IFNy inhibition and recombinant IFNy treatments}

Neutralization of IFN $\gamma$ activity was achieved by treating mice with i.p. injections of 0.5mg anti-IFN $\gamma$ monoclonal antibody (clone XMG1.2) (InVivoMAb) suspended in $0.2 \mathrm{ml}$ sterile saline 8 hours before $\mathrm{Lm}$ infection and 1- and 2- days post $\mathrm{Lm}$ infection. Treatment was only given to Lm only groups, while a separate Lm only and coinfected groups were given i.p. injections of $0.2 \mathrm{ml}$ sterile saline. In contrast, $0.5 \mathrm{mg}$ of recombinant IFN $\gamma$ (Abcam, Cambridge, MA) was given to coinfected mice in 3 separate i.p. injections in $0.2 \mathrm{ml}$ sterile saline 8 hours before $\mathrm{Lm}$ infection and also 1- and 2- days post Lm. 


\section{Splenic myeloid cell analysis}

Spleens were removed on the indicated days post Py infection or from agematched naïve mice and placed in HyClone RPMI 1640 (Thermo Fisher Scientific, Waltham, MA) supplemented with 10\% fetal bovine serum (Atlanta Biologicals, Lawrenceville, GA), $1.19 \mathrm{mg} / \mathrm{ml}$ HEPES (Corning, New York, NY), $0.2 \mathrm{mg} / \mathrm{ml} \mathrm{L-}$ glutamine (Research Products International, Mt. Prospect, IL), 0.05M 2-mercaptoethanol (Thermo Fisher Scientific), $0.05 \mathrm{mg} / \mathrm{mL}$ penicillin/streptomycin (Invitrogen, Grand Island, NY), and $0.05 \mathrm{mg} / \mathrm{mL}$ gentamicin (Corning). Single cell suspensions were treated with ammonium chloride potassium solution $\left(0.15 \mathrm{mM} \mathrm{NH}_{4} \mathrm{Cl}, 1.0 \mathrm{mM} \mathrm{KHCO} 3,0.1 \mathrm{mM}\right.$ $\mathrm{Na}_{2}$ EDTA, $1 \mathrm{~N} \mathrm{HCl}$ ) to lyse red blood cells and then washed in RPMI then resuspended in FACS buffer (1XPBS, 1\%FBS, 0.02\% sodium azide) containing Fc block (antiCD16/32; clone 2.4G2). Splenocytes were then stained with cell surface marker-specific antibodies. Anti-CD3€ FITC (clone 145-2C11), anti-CD19 FITC (clone 6D5), antiCD11b PE/Cy7 (clone M1/70), anti-CD11c BV650 (clone N418), anti-Ly-6G BV421 (clone 1A8), anti-Ly-6C PerCP/Cy5.5 (clone AL-21), anti-F4/80 PE Dazzle 594 (clone BM8) were from Biolegend (San Diego, CA). Anti-CD209b APC (clone eBio 22D1), anti-CD169 (clone SER-4) were from eBiosciences (San Diego, CA). Anti-SIGLEC-F APC R700 (clone E50-2240) was from BD Pharmingen (San Diego, CA). Cells were incubated with antibodies at $4^{\circ} \mathrm{C}$ for 15 minutes. Cells were then fixed in $4 \%$ paraformaldehyde fixation buffer (Biolegend) and then washed three times in FACS buffer before being analyzed on a BD LSRFortessa ${ }^{\mathrm{TM}}$. 


\section{Spleen phagocytic cell isolation and intracellular bacterial survival assay}

Single cell suspensions from 3 naïve or Py infected mice were generated as described above and pooled together. Splenic phagocytes were enriched by negative depletion of $\mathrm{CD}^{+}$and $\mathrm{CD} 19^{+}$cells using anti-CD3 $\epsilon$ and anti-CD19 microbeads (Miltenyi Biotec, Bergisch Gladbach, Germany), according to manufacturer recommendations. $\mathrm{CD}^{-}$and $\mathrm{CD}^{-}{ }^{-}$flow through cells were stained with anti-CD11b PE/Cy7 (clone M1/70) and CD11c APC (clone N418) from Biolegend (San Diego, CA). CD11b ${ }^{\text {hi }} / C D 11 c^{-}$cells were sort purified with a BD FACSAria ${ }^{T M}$ III. Sorted cells were plated and incubated at $37^{\circ} \mathrm{C}$ with $5 \% \mathrm{CO}_{2}$ for 1 hour. For some experiments, cells from naïve mice were incubated for 30 minutes with $30 \mu \mathrm{g}$ Py hemozoin $(\mathrm{PyHz})$. Sorted cells were infected with Lm that had been opsonized by incubating in RPMI with $10 \%$ homologous mouse serum (naïve cells + Lm opsonized with naïve serum; Py cells + Lm opsonized with Py serum) rotating for 30 minutes at $37^{\circ} \mathrm{C}$. Bacteria was washed once in RPMI and cells were infected with a multiplicity of infection $(\mathrm{MOI})=100$. One hour after the infection, media was removed from cells and replaced with RPMI containing $5 \mu \mathrm{g} / \mathrm{mL}$ gentamicin to kill extracellular Lm. Intracellular Lm burden was determined after 1 hour and 4 hours of incubation in RPMI with gentamicin by removing the media containing gentamicin and resuspending the cells in sterile deionized $\mathrm{H}_{2} \mathrm{O}\left(\mathrm{dH}_{2} \mathrm{O}\right)$ to lyse cells and release surviving Lm. Fold change in intracellular Lm was determined by dividing cfu at 4 hours post infection by cfu/well at 1 hours after the addition of gentamicin. 


\section{Microscopy}

Cells were from either peripheral blood, obtained through tail snip, or spleen or liver single cells isolated and passed through a 100-micron pore size nylon mesh. In some experiments, CD11 bi $/$ CD11 $c^{-}$cells were sort purified from T cell and B cell depleted spleen samples. Blood samples were smeared on a glass slide and allowed to dry, spleen and liver cells were placed onto a glass slide in a drop of RPMI containing $15 \%$ mouse serum and incubated at $37^{\circ} \mathrm{C}$ with $5 \% \mathrm{CO}_{2}$ for 30 minutes to allow cells to adhere. For all samples, cells were fixed in methanol for 20 minutes and stained with Giemsa dye (Azer Scientific, Morgantown, PA) for 20 minutes and rinsed with $\mathrm{ddH}_{2} \mathrm{O}$. In some cases, cells were incubated with $\mathrm{Hz}$ as described above and $\mathrm{Hz}$ is visible as brown pigment. Images were obtained with a Nikon eclipse Ci light microscope at 100X magnification or a Zeiss Axio Imager .A1 microscope with an AxioCam MRm digital camera (Zeiss) at 63 or 96X in oil emersion. Scale bars were estimated based on average diameter of murine erythrocytes (Nikon) or with Axiovision SE64 imaging software (Carl Zeiss Microscopy, LLC, White Plains, NY).

\section{Isolation of P. yoelii-derived hemozoin (PyHz)}

Livers and spleens were obtained from mice previously infected with Py after clearance of the parasite infection, between 30- and 65-days post-infection. Organs were collected and homogenized with a tissue homogenizer in de-ionized $\mathrm{H}_{2} \mathrm{O}\left(\mathrm{dH}_{2} \mathrm{O}\right)$ and kept on ice for 1 hour. Homogenates were centrifuged at $3100 \mathrm{x}$ g for 10 minutes. Pellets were washed twice in $\mathrm{dH}_{2} \mathrm{O}$ and passed through 100-micron nylon mesh (ELKO Filtering Co.). Flow-through was collected, washed and resuspended in sterile PBS before being 
added to the top of a discontinuous Percoll density gradient containing $40 \%(1.07 \mathrm{~g} / \mathrm{mL})$ and $80 \%(1.12 \mathrm{~g} / \mathrm{mL})$ fractions. Gradients were centrifuged at 5,000 x $\mathrm{g}$ for 20 minutes with slow acceleration/deceleration at $4^{\circ} \mathrm{C}$. Hemozoin from Py infected mouse organs (PyHz) was collected from the interphase between the $40 \%$ and $80 \%$ fractions, as well as from the dark pellet beneath the $80 \%$ fraction. Samples were washed twice in $\mathrm{dH}_{2} \mathrm{O}$ and finally resuspended in sterile PBS. Purification of ‘clean' PyHz was performed for some experiments with a method adapted from Coban et al. [82], where organ homogenate was lysed in $0.2 \%$ IGPAL and washed 3-4 times in $2 \%$ sodium dodecyl sulfate (SDS) and then incubated overnight in $10 \mathrm{mM}$ Tris- $\mathrm{HCl}(\mathrm{pH} 8.0), 0.5 \% \mathrm{SDS}, 1 \mathrm{mM} \mathrm{CaCl}_{2}$, and $2 \mathrm{mg} / \mathrm{mL}$ proteinase $\mathrm{K}$ at $37^{\circ} \mathrm{C}$. Next, samples were washed again in $2 \% \mathrm{SDS}$ and then incubated at $37^{\circ} \mathrm{C}$ for 3 hours in $6 \mathrm{M}$ urea, washed in $1 \mathrm{X}$ PBS and resuspended in saline for experimental use. For quantification of $\mathrm{Hz}$ in mouse tissues, organs were homogenized and incubated in $1 \%$ Proteinase K (VWR, Solon, $\mathrm{OH}$ ) overnight at $37^{\circ} \mathrm{C}$. Hz was quantified using a protocol adapted from Deroost, et al., 2012 [83]. Briefly, Hz was centrifuged at $3100 \mathrm{x}$ g and pellets were washed three times in $100 \mathrm{mM} \mathrm{NaHCO} 3, \mathrm{pH}$ 9.0 with $2 \%$ SDS. Hz, and a dilution series of synthetic hemozoin (InVivoGen, San Diego, CA) of known concentration, were resuspended in 100mM NaOH, 2\% SDS, 3mM EDTA and then sonicated for 5 minutes before incubating for 30 minutes at room temp to dissolve hemozoin into soluble heme. Quantification was determined by reading absorbance at $\mathrm{OD}_{400}$ and comparing values of samples prepared from Py-infected mouse organs to the synthetic $\mathrm{Hz}$ standards. For some experiments, $\mathrm{PyHz}$ was incubated at $37^{\circ} \mathrm{C}$ in $100 \mathrm{U} / \mathrm{mL}$ DNase with $1 \mathrm{mM} \mathrm{CaCl} 2$ and $2 \mathrm{mM} \mathrm{MgCl}_{2}$ for 2 hours. These samples were then washed twice in $2 \mathrm{mM}$ mannitol-containing phosphate buffer and resuspended in 
sterile saline. Absence of DNA in PyHz sample was verified by staining with GelRed Nucleic Acid stain and running samples on 1\% agarose gel.

\section{Plasmodium falciparum culture and preparation of hemozoin}

Natural P. falciparum hemozoin (PfHZ) was prepared from in vitro human erythrocytes cultures infected with $P$. falciparum (Palo Alto strain, mycoplasma-free) treated with DNase (Sigma, St. Louis, MO). Contamination with endotoxin (LPS) was excluded by E-Toxate assay (Sigma, St. Louis, MO), as previously described [84]. Briefly, synchronized parasite cultures were supplemented with DNase (5 U/ml) 12-15 hours previous to schizogony. PfHZ which was naturally expelled during schizogony, was harvested at the $25 / 40 \%$ interface of a discontinuous percoll gradient, incubated with 40U/ml DNase, $2 \mathrm{mM} \mathrm{MgCl} 2$ and $1 \mathrm{mM} \mathrm{CaCl}_{2}$ in $\mathrm{PBS}$ at $37^{\circ} \mathrm{C}$ for $1 \mathrm{~h}$ and subsequently washed several times in a $10 \mathrm{mM}$ phosphate buffer ( $\mathrm{pH} 8.0$ ) containing $2 \mathrm{mM}$ mannitol. $\mathrm{HZ}$ was quantified by its heme content using a luminol-enhanced luminescence assay as previously described [85].

\section{In vivo $\mathrm{Hz}$ treatments}

Mice were treated with $1-1.5 \mathrm{mg}$ of the indicated preparations of $\mathrm{Hz}$ intravenously by injection through the tail vein, or retro-orbital vein. Prior to injection, Hz was washed twice from storage solution, 1X PBS with 2mM Mannitol and 20mM HEPES with 1X PBS and resuspended in sterile saline. The entire volume of the final $\mathrm{Hz}$ preparation was then passed 3 to 5 times through a series of syringe needles with $21,25,27.5$, and 30 1/2 gauges. Mice were then slowly and carefully injected with 200 to $300 \mu \mathrm{lof} \mathrm{Hz}$ to avoid 
cardio-pulmonary distress. In some cases, mice received 2 injections to achieve a total of 1-1.5mg on consecutive days. Lm or Sp infections were initiated 7 days after $\mathrm{Hz}$ infections

\section{Reactive oxygen species assay}

Spleens and blood (for opsonizing bacteria) were collected from naïve control and Py-infected mice (days 10-16 post infection). Spleens were processed to generate single cell suspensions. Cells were plated at $2 \times 10^{6}$ cells/well resuspended in RPMI with $10 \%$ fetal calf serum incubated at $37^{\circ} \mathrm{C}$ for 30 minutes with $5 \% \mathrm{CO}_{2}$. Cells were then incubated with the following antibodies; anti-CD3€ APC (clone 145-2C11), anti-CD19 APC (clone 6D5), anti-CD11b PE/Cy7 (clone M1/70), anti-Ly-6G BV421 (clone 1A8), and 2',7'-dichloro-hydrofluorescein diacetate (DCF; Abcam Cambridge, MA) for 20 minutes. Cells were then left untreated or treated for 20 minutes at $37^{\circ} \mathrm{C}$ with $5 \% \mathrm{CO} 2$ with $20 \mu \mathrm{M}$ phorbol 12-myristat 13-acetate (PMA), unopsonized Lm or Sp, naïve serum opsonized Lm or Sp, or Py serum opsonized Lm or Sp at an MOI of $10 \mathrm{Sp}$ was heat treated (Ht Sp) at $65^{\circ} \mathrm{C}$ for 1 hour. Lm and $\mathrm{Ht}$ Sp were opsonized in $10 \%$ serum, from naïve or Py-infected mice, in RPMI while incubating at $37^{\circ} \mathrm{C}$ and rotating. After treatment, cells were centrifuged at $300 \mathrm{x}$ g for 5 minutes then incubated at $37^{\circ} \mathrm{C}$ for 20 minutes followed immediately by analysis on a BD LSRFortessa ${ }^{\mathrm{TM}}$.

\section{Statistical analysis}

Data were analyzed as indicated in each figure legend using GraphPad Prism v7. 


\section{CHAPTER II}

\section{CHARACTERIZATION OF MALARIA AND BACTERIAL COINFECTIONS}

\section{Introduction:}

An association between malaria and increased susceptibility to invasive bacterial disease has been recognized by several clinical reports. With the exception of emerging insight into mechanisms by which malaria increases susceptibility to NTS, there is still little known about the underlying mechanisms by which Plasmodium infection suppresses host antibacterial immunity. In regard to NTS, Plasmodium-induced hemolysis results in the activation of heme oxygense-1 (HO-1), which mobilizes functionally immature granulocytes from the bone marrow. These immature granulocytes exhibit impaired production of reactive oxygen species in response to PMA stimulation, and decreased killing of NTS [62]. Similar impairments to NTS immunity were observed when mice were treated with phenylhydrazine to induce hemolysis or hemin to mimic hemolysis, leading to the conclusion that impaired bacterial immunity during Plasmodium infection is attributed to hemolysis with no parasite-specific factor contributing to the immunosuppression [61]. Additionally, Plasmodium-induced IL-10 from myeloid cells suppresses the ability of myeloid cells to control NTS, resulting in increased systemic colonization in co-infected mice [59]. Moreover, IL-10 signaling 
through the IL-10 receptor can directly induce HO-1 to suppress anti-bacterial immunity, creating an environment that favors intracellular growth and dissemination of NTS [86].

Streptococcus pneumoniae (Sp), also known as "Pneumococcus", is a commensal bacterium in the human nasopharynx, but is capable of causing disease when symbiosis between the bacterium and host is disrupted. A systemic review of pneumococcal carriage in sub-Saharan Africa found children under 5 years old had approximately $60 \%$ carriage, while children 5-15 years old had about $40 \%$ carriage [87]. When symbiosis between the host and $\mathrm{Sp}$ is disrupted, Sp can cause a variety of infections including; pneumonia, sinusitis, otitis media, bacteremia/sepsis, and meningitis [88]. Importantly, pneumococcus has been identified as one of the most common pathogens isolated from blood samples of children in Africa with invasive bacterial infections [45].

While clinical studies do not unanimously agree that $\mathrm{Sp}$ is frequently concomitant with malaria, several features of these studies may be limiting Sp detection. For example, one study that presented low abundance of Sp detected in blood of children with malaria excluded patients that showed signs of respiratory tract illness, a symptom that is commonly associated with malaria and MA-ARDS and would be likely to occur in a patient with invasive pneumococcal disease [46]. The same study reported a high amount of known contaminant organisms, such as Staphylococcus aureus, which accounted for $43 \%$ of all bacteria isolated from blood cultures, compared to $3 \% \mathrm{Sp}$. Other factors that could have decreased Sp detection are its fastidious nature in culture, tendency to autolyze during static growth conditions, and many diagnostic labs may not have the resources on hand to examine Gram stains, colony morphology, and hemolysis phenotype [89]. Contrary to these potentially problematic reports, several broad scale 
clinical reviews have shown that $\mathrm{Sp}$ is among the bacterial infections most commonly found in association with malaria in children (Reddy et al., Churchet al.) and adults (Aumaet al., Morton et al., Nadjm et al., and Reddy et al.) [43-45, 53, 90]. Moreover, an analysis of Mozambican children in a rural hospital identified Sp as the leading pathogen associated with malarial anemia and severe malaria [91]. In contrast to NTS, to our knowledge there are no mechanistic studies that have assessed the interaction between malaria and Sp coinfection, and no animal models of malaria and Sp infection have been described. To this end, I have developed a malaria and Sp coinfection mouse model. The outcome of malaria depends in large part on the timing and magnitude of cytokine production, and the balance of inflammatory and immune suppressive responses induced by Plasmodium infection. There is a well-known correlation between the severity of malarial disease and high levels of systemic IFN $\gamma, \mathrm{TNF} \alpha$ and other proinflammatory cytokines [92-94]. Ideally, these cytokine signals work synergistically to orchestrate the production of anti-parasitic factors like NO and other radicals [95]. To balance these pro-inflammatory responses, the host induces anti-inflammatory cytokines, in particular TGF- $\beta$ and IL-10. Yet, elevated levels of these anti-inflammatory cytokines can also lead to increased mortality and hyperparasitemia [96-98].

While cytokines play a major role in both innate and adaptive immune responses during malaria, IL-10 activity during malaria in particular has garnished attention as a potential contributor to Plasmodium-induced suppression of immunity to non-typhoid Salmonella $[59,99]$. Observations have been made in laboratory animal studies and human clinical studies that show an IL-10-dependent inhibition of neutrophil function and recruitment to infected tissues during acute and recently cleared Plasmodium 
infections, making a clear case for IL-10 in suppressing immunity to bacterial coinfections. Experiments conducted by the laboratory of Renee Tsolis found that $P$. yoelii-infected IL-10 deficient mice as well as $P$. yoelii-infected mice treated with antiIL-10 antibody to block activity had decreased systemic levels of non-Typhoid Salmonella following the secondary infection [59]. Alternatively, the Tsolis group found mice treated with recombinant IL-10 elevated bacterial burdens similar to both P. yoeliiinfected mice and mice with anti-RBC antibody treatment-induced anemia [59]. The connection between IL-10, anemia, and Plasmodium infection also involves heme oxygenase-1, an enzyme also linked to increased susceptibility to non-Typhoid Salmonella during malaria - whereby both IL-10 and free heme during anemia can independently induce HO-1 [62]. This interaction that renders a host with limited antiSalmonella immunity is fundamentally linked to parasite-driven hemolysis during bloodstage malaria, where the continuous rupture of schizonts from infected erythrocytes leads to anemia. The physiological effects of anemia eventually normalize as homeostasic concentration of $\mathrm{RBCs} / \mathrm{mL}$ blood occurs within 10 days of parasite clearance from circulation [62]. Together, these mechanisms are thought to impair neutrophil functions, recruitment and activation of innate immune cells, thereby providing an environment which favors the growth of Salmonella and systemic spread of invasive infection.

Since the overall goals of this work relate to the impact of Plasmodium infection on immunity to bacterial coinfection, it is critical to consider whether factors that are known to be involved in the heightened susceptibility to Salmonella during malaria are also important in other bacterial coinfections. The limitation of malaria-bacterial coinfection mechanistic studies to Plasmodium and NTS creates a knowledge gap as it is 
unknown whether the mechanisms of Plasmodium-driven immune suppression are specific to NTS or if they will apply broadly to all invasive bacterial infections. Furthermore, no parasite-specific factor has been identified to contribute towards Plasmodium-induced impaired immunity to bacterial coinfections. These represent important knowledge gaps that impede treatment of bacteria coinfections during malaria.

The focus of this chapter is dedicated to testing the nature of Plasmodiumbacterial coinfections other than NTS, and also determine whether the same hemolysisdependent mechanisms found to impair immunity to NTS are involved. While some progress has been made in exploring the factors involved in Plasmodium-induced susceptibility to NTS infection, there is a critical need for additional animal model systems to investigate other coinfections with malaria. One of the overall aims of this work is to provide a model for Plasmodium-Pneumococcal coinfection which can be used to identify factors associated with the increased risk of invasive pneumococcal bacteremia/sepsis and where treatments and therapies can be designed and tested. This chapter will describe two models of murine Plasmodium-bacterial (Listeria monocytogenes, and Streptococcus pneumoniae) coinfection used to examine the mechanisms by which innate immunity to bacterial infections is impaired during malaria. Using these models, I assessed the ability of IL-10 and HO-I activation to determine if these pathways that contribute to impaired control of NTS during malaria, also impact control of other bacterial infections. Finally, the duration of Plasmodium-driven suppression of anti-bacterial innate immunity is explored. 


\section{$\underline{\text { Results: }}$}

Plasmodium yoelii infection results in long-term suppression of innate immunity during systemic bacterial coinfection

In order to investigate Plasmodium-infection related immunosuppression and subsequent impaired immunity to bacterial coinfections, we began probing immune responses using a model bacterium, Listeria monocytogenes (Lm) $\Delta$ actA mutant strain, which is attenuated due to the deletion of the virulence factor gene that allows actin polymerization-dependent intracellular mobility and the ability to spread from cell to cell [100]. While Lm is not a common bacterial infection seen in coinfections during malaria, it is useful due to the vast amount of knowledge about its interaction with innate and adaptive immune responses. Thus, the creation of a malaria-bacteria coinfection model with Lm provided an insightful means to probe a predictable and well-characterized infection system. The readout of bacterial burdens in form of colony forming units (cfu) from spleens and livers at time points ranging 1 to 3 days post Lm infection presents a quantitative way to evaluate the quality of the innate immune response to a bacterial infection.

To examine Plasmodium-induced suppression of innate immunity during systemic bacterial infection C57BL/6N mice were first infected with Plasmodium yoelii 17XNL (Py), which resulted in increasing parasitemia through about three-weeks post infection that was followed by resolution of the infection during the ensuing week (Fig. 4A). Control and Py-infected mice were subsequently infected, via intravenous injection, with Lm $\Delta a c t A 7$-days post Py infection. Clearance of Lm was delayed, in particular in the spleen, in Py-infected mice compared to control mice (Fig. 4B). To examine Py-induced 
suppression over the course of Py-infection, mice were infected with $\operatorname{Lm}$ on day 7, 14,

and 28 post-Py infection and spleens were removed 2-days later to quantify Lm burden.

Throughout the course of Py infection, mice had elevated bacterial burdens (Fig. 4C).

Remarkably, impaired innate immunity to Lm was also observed at both one-month (day

63) and two-months (day 98) post-Py infection (Fig. 4C). Collectively, these data

demonstrate that Py suppresses the innate immune response during systemic bacterial

infection with Lm, including time points well beyond resolution of the Py infection.
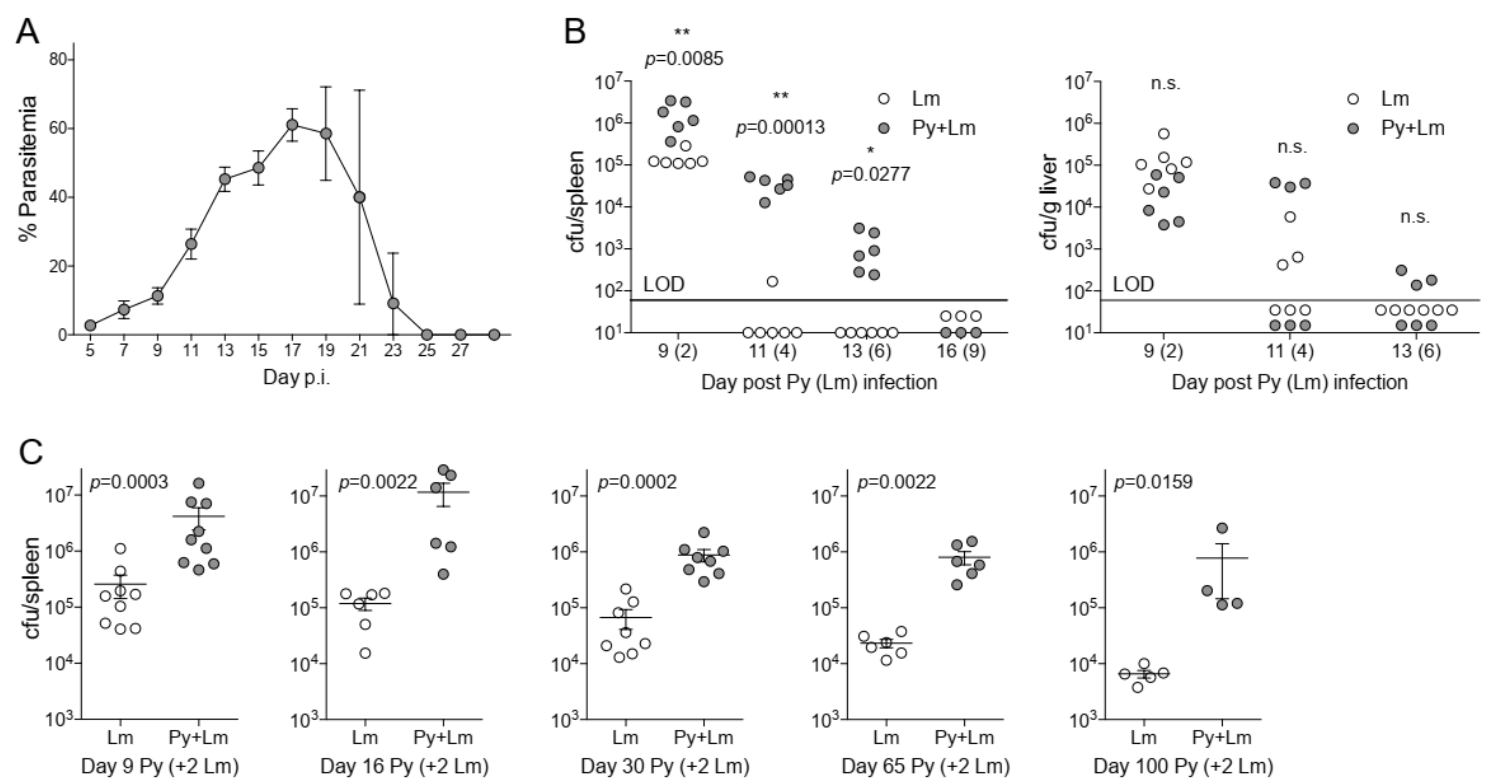

Figure 4. $P$. yoelii infection induces long-term suppression of anti-bacterial innate immunity during bacteremia. A) C57BL/6N mice were infected with $10^{5}$ Py pRBCs. Percent parasitemia (percent of RBCs infected with Py) was evaluated during the course of infection. Data (mean \pm S.D.) are from 9 mice and representative of 3 independent experiments. B) C57BL/6N mice were infected with $10^{5} \mathrm{Py}$ pRBCs, seven days later both control and Py-infected mice were infected with $5 \times 10^{6} \mathrm{CFU} \mathrm{Lm} \triangle \mathrm{ActA}$. Lm burden was determined in the spleen and liver on days 2, 3, 6, and 9 post-Lm infection. Data are cumulative results from two-independent experiments with 3 or 6 mice per group per time point. Each symbol represents an individual mouse. Data were analyzed by two-tailed Fisher's exact test. LOD $=$ limit of detection. [cfu/spleen day 11 (4) data was originally published in The Journal of Immunology. White C.E., Villarino N.F., Sloan S.S., Ganusov V.V., and Schmidt N.W. 2015. Plasmodium Suppresses Expansion of T Cell Responses to Heterologous Infections. J. Immunol. 194: 697-708. Copyright () 2015 The American Association of Immunologists, Inc.] C) C57BL/6N mice were infected with $10^{5}$ Py pRBCs. On days 7, 14, 28, 63, and 98 post-Py infection both Py-infected and control mice were infected with 5x106 CFU Lm.

Bacterial burdens were determined 2 days post $\mathrm{Lm}$ infection. Data (mean \pm S.E.) are cumulative results from 2- to 3-independent experiments with 4 to 9 mice per group. Data were analyzed with Mann-Whitney test, n.s., not significant. 
Py infection impairs IFNy production in response to $\triangle a c t A$ Lm during parasitemia, but

enhances Lm induction of IFNy after parasite clearance.

IFN $\gamma$ is an important inflammatory cytokine produced during Plasmodium infections that activates innate immune cells to enhance anti-parasite functions, such as uptake and killing of infected RBCs and enhance production of other inflammatory signals to amplify the immune response [101, 102]. There is an early burst of IFN $\gamma$, produced primarily by natural killer T-cells (NK), and to a lesser degree, $\mathrm{CD} 4^{+} \mathrm{T}$ cells, in the early days of a blood-stage infection [103, 104]. There is also an induction of immunosuppressive factors such as IL-10 that functions to prevent immunopathology associated with the production of inflammatory cytokines, which can also suppress the overall immune response. It is also well established that IFN $\gamma$ signaling is paramount in initiating an effective immune response to Lm infection [105].

To test whether Py infection impairs the production of IFN $\gamma$ in response to secondary $\triangle$ actA Lm infection, mice were either challenged with Py followed by Lm 7 days later or only $\mathrm{Lm}$. Serum was collected from peripheral blood before $\mathrm{Lm}$ infection (day 7 post Py) and both spleens and serum were collected 24 hours after i.v. Lm infection. Prior to Lm infection, baseline levels of IFN $\gamma$ were detected in either coinfected or Lm only infected mice. One day after Lm infection, however, coinfected mice had significantly lower levels of IFN $\gamma$ in both whole spleen homogenate and in serum (Fig. 5 A).

Since we already observed that Py-infected mice had an impaired ability to eliminate Lm infection up to 2 months after parasite clearance (Fig. 4C), we sought to examine whether or not IFN $\gamma$ production in response to $\mathrm{Lm}$ was reduced in coinfected 
mice at this late timepoint post Py infection. Unlike the Py-induced suppression of IFN $\gamma$ seen when Lm infection was initiated 7 days post Py, when Lm infections began 100 days post Py, levels of IFN $\gamma$ in serum of coinfected mice were significantly higher than mice receiving only Lm both 1 and 2 days after Lm infection. A similar trend was seen in spleens 2 days post $\mathrm{Lm}$ (Fig. 5B). These results suggest that although IFN $\gamma$ production is limited in Py-infected mice during acute parasitemia, the response to Lm infection becomes primed at later timepoints and able to produce a more robust IFN $\gamma$ burst.

In order to determine the degree to which the lack of IFN $\gamma$ production contributed to the inability of coinfected mice to clear Lm at early time points, IFN $\gamma$ activity was blocked in coinfected mice and mice infected with Lm only. C57BL/6 mice were infected with Py and given i.p. treatments of either anti-IFN $\gamma$ antibody or isotype control 8 hours prior to Lm infection and additional treatments 24 - and 48 -hours post Lm. Spleens and livers from these mice were then collected 3 days post Lm (day Py+10). Analysis of bacterial burdens showed that blocking IFN $\gamma$ in Lm only mice did not impair clearance from spleens (Fig. 6C). In an alternative approach, coinfected mice received treatments of recombinant IFN $\gamma$ via i.p. injection 8 hours prior to Lm infection on day 7 post Py as well as 24- and 48-hours post Lm. Spleens and livers from these mice were then collected 3 days post Lm (day Py+10), while coinfected and Lm only infected mouse groups received no treatment. Treating coinfected mice with recombinant IFN $\gamma$ did not improve Lm clearance in the spleen or liver (Fig. 4D). In a single experiment (shown in Figure 5E), coinfected mice given wildtype Lm did not appear to have as significant of a decrease in serum IFN $\gamma 1$ day after $\mathrm{Lm}$ infection compared to what was observed with in the Py coinfection with the $\triangle a c t A$ mutant (Fig. 5A and B) and had 
similar IFN $\gamma$ levels as mice that received wildtype Lm alone by 2 day post Lm infection (Fig 5E). Together, these results do not support the hypothesis that the Py-infection induced decrease in IFN $\gamma$ production in response to $\mathrm{Lm}$ infection plays a major role in the delayed Lm clearance that we have observed. 
$\bar{A}$.

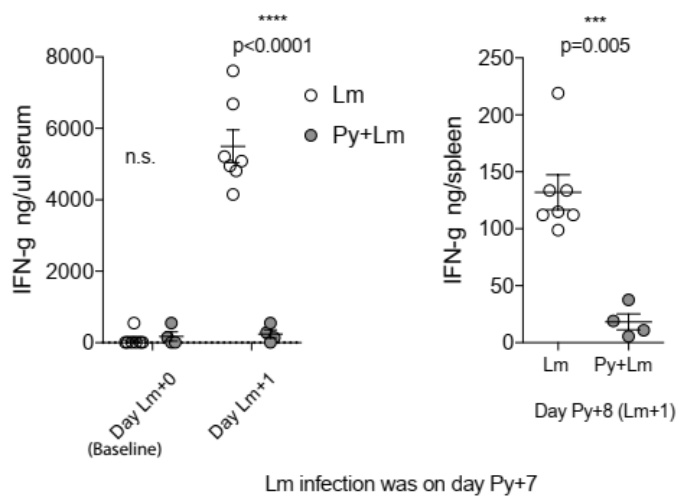

C.
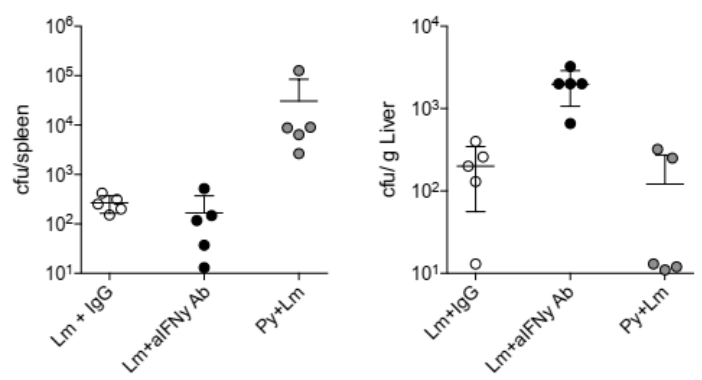

B.
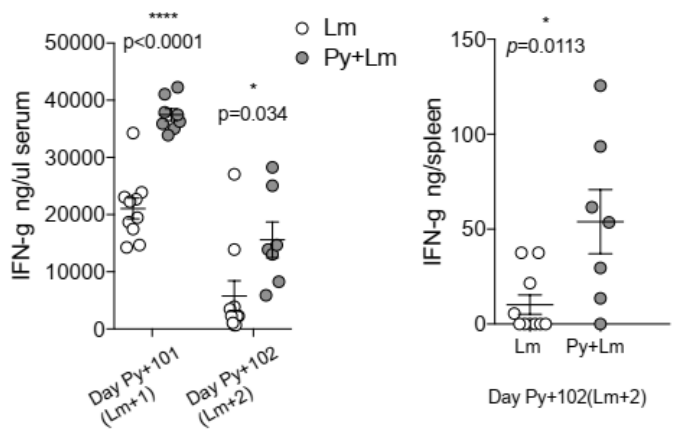

Day Py+102(Lm+2)

D.
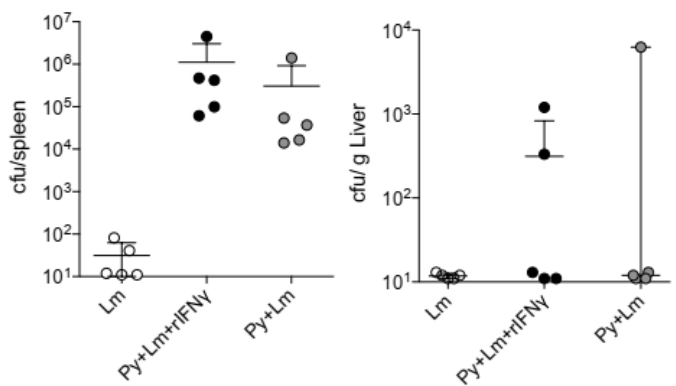

Figure 5. IFN $\gamma$ production during early and late coinfection. (A,C-D)C57BL6 mice were infected with $10^{5} \mathrm{Py}$ pRBCs followed by $5 \times 10^{6} \mathrm{cfu} \mathrm{Lm}$ on days indicated in figure. A) Serum was collected via retroorbital bleeds 7 days post Py infection, prior to $\triangle a c t A \mathrm{Lm}$ infection, and both serum and spleens were collected 24 hours after Lm infection (day Py+8). B and E) Mice were infected with Lm 100 days post Py infection, or Lm wt on day Py+7. Serum was collected 1- and 2-days post Lm and spleens were harvested 2 days post $\mathrm{Lm}($ day $\mathrm{Py}+102) \mathrm{B})$, day $\mathrm{Py}+9 \mathrm{E})$. IFN $\gamma$ concentrations from serum and spleen homogenate were determined with ELISA. C) Mice were treated with anti-IFN $\gamma$ monoclonal antibody 8 hours prior to $\mathrm{Lm}$ infection and 1- and 2-days post Lm D) Mice were treated with recombinant IFN $\gamma 8$ hours prior to and 1- and 2- days after Lm infection. C-D) or wildtype Lm (E). Tissues were collected for cfu analysis day 3 post Lm Data are mean \pm S.D. (A, C,-D) and are from a single independent experiment that represent 2-3 repeated experiments. Data in B. are mean \pm S.E. and are cumulative results from 2 independent experiments. Data in E are mean \pm S.D from only one experiment. Statistics are One-Way ANOVA with Kruskal-Wallis multiple comparisons test or unpaired t-tests. 


\section{Py infection improves clearance of wild-type Lm with functional actin polymerization}

\section{$\underline{\text { ability }}$}

Our initial observations with the Py+Lm coinfection model were made with an attenuated mutant strain of Lm that is lacking the actA gene, which is involved in the ability of Lm to polymerize actin and is a major virulence factor that allows Lm to invade nearby cells and evade the immune system $[100,106]$. One advantage of using the attenuated $\Delta a c t A$ mutant was due to the higher inoculum that can be delivered to mice through intravenous injection without producing a lethal infection, allowing injections of $5 \times 10^{6} \mathrm{cfu}$ rather than $10^{4} \mathrm{cfu}$ of wildtype $\mathrm{Lm}$ that is consistently lethal in C57BL/6 mice [107]. In order to determine whether the impaired clearance of Lm following Py infection was dependent on a functional actA gene in Lm, C57BL/6 mice were infected with Py followed by infection with $10^{4} \mathrm{cfu}$ i.v. wildtype Lm 10403S 7 days later. Control mice received only the wildtype Lm infection. Two days after bacterial infection spleens and livers were collected from Lm only and Py+Lm coinfected mice and bacterial burdens were assessed. Surprisingly, when using virulent Listeria, coinfected mice had drastically lower bacterial burdens in the spleen than mice that received wildtype Lm alone (Figure 5A). While these results raised interesting questions pertaining to Listeria biology and how the ability to polymerize actin and escape from the intercellular space of a cell or invade neighboring cells impacts outcome of Listeria during coinfection with Py, these ideas were not further explored. 


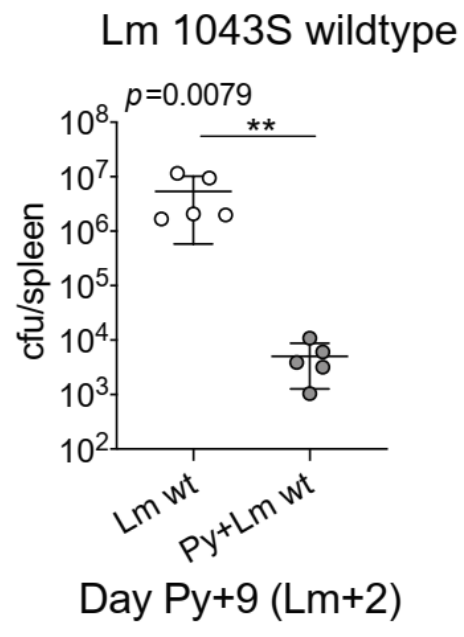
Figure 6. Py infection confers protection against wildtype Lm. C57BL/6 mice were infected with $10^{5} \mathrm{Py}$ pRBCs followed by $5 \times 10^{6}$ cfu wildtype Lm 7 days later. Serum was collected 24 - and 48- hours post Lm infection via retro-orbital bleed and spleens were collected 2 days post Lm Data (mean \pm S.D.) are from a single independent experiment that represent 3 independent experiments. Statistical analysis was performed with a Mann-Whitney U test.

\section{Contribution of P. yoelii-induced HO-1 and IL-10 to impaired innate immunity is}

\section{bacteria-dependent}

Previous studies have identified mechanisms by which Plasmodium suppresses immunity to NTS $[59,61,62,108,109]$. Currently, nearly all mechanistic investigations of impaired immunity to non-Typhoid Salmonella (NTS) coinfections during malaria have built upon the key findings that the activities of HO-1 and IL-10, which are produced during active Plasmodium infection, play an important role in the increased severity of bacterial infection $[59,62]$. Plasmodium infections result in the release of heme, which activates HO-1. HO-1 induces the release of immature granulocytes that exhibit impaired oxidative burst in response to PMA stimulation [61]. Additionally, Plasmodium infections induce myeloid cells to produce IL-10 that impairs control of NTS infection [59]. To determine whether those mechanisms of impaired immunity to bacterial coinfections are specific to NTS coinfections, we first confirmed the results of those studies by infecting mice with $P$. yoelii followed by coinfection with NTS. As shown before, P. yoelii-infected mice treated with tin protoporphyrin IX (Sn PP; a 
synthetic heme analog that inhibits activity of HO-1) or an anti-IL-10 monoclonal antibody had reduced bacterial burdens compared to the respective control treated mice (Fig.7 A and B). Contrary to observations in NTS coinfections, Sn PP treatment did not improve anti-bacterial immunity in Py+Lm (Fig. 7C) co-infected groups, as bacterial burdens of Sn PP treated mice were comparable to control treated groups. Blocking IL-10 with an anti-IL-10 monoclonal antibody treatment prior to Lm infection also did not improve bacterial clearance in co-infected mice (Fig. 7D). These data show that our use of Sn PP and anti-IL-10 antibodies to block activity of HO-1 and IL-10, respectively, is valid in our model of Py bacterial coinfections since we have shown the same trend as Cunnington et al. replicated data from Lokken et al. with the same strain of NTS [59, 62]. The data also show that unlike in NTS coinfections with Py, HO-1 and IL-10 do not play a role in the impaired antibacterial immunity in Lm+Py coinfections. Together, this suggests that there may be other mechanisms by which Plasmodium infection induces defective immune responses to bacterial coinfections. 
A

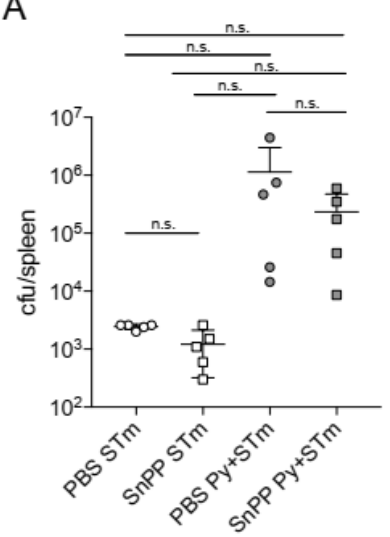

B

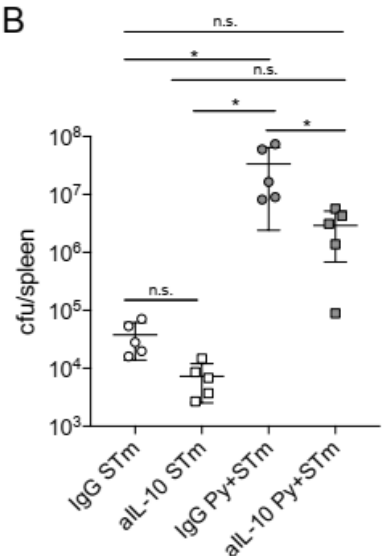

C

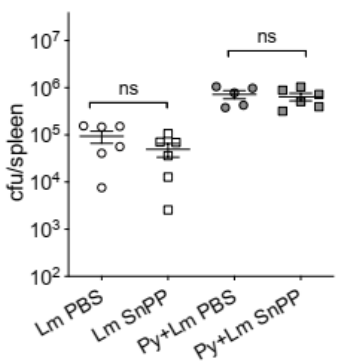

Day 9 Py (+2 Lm) p.i.
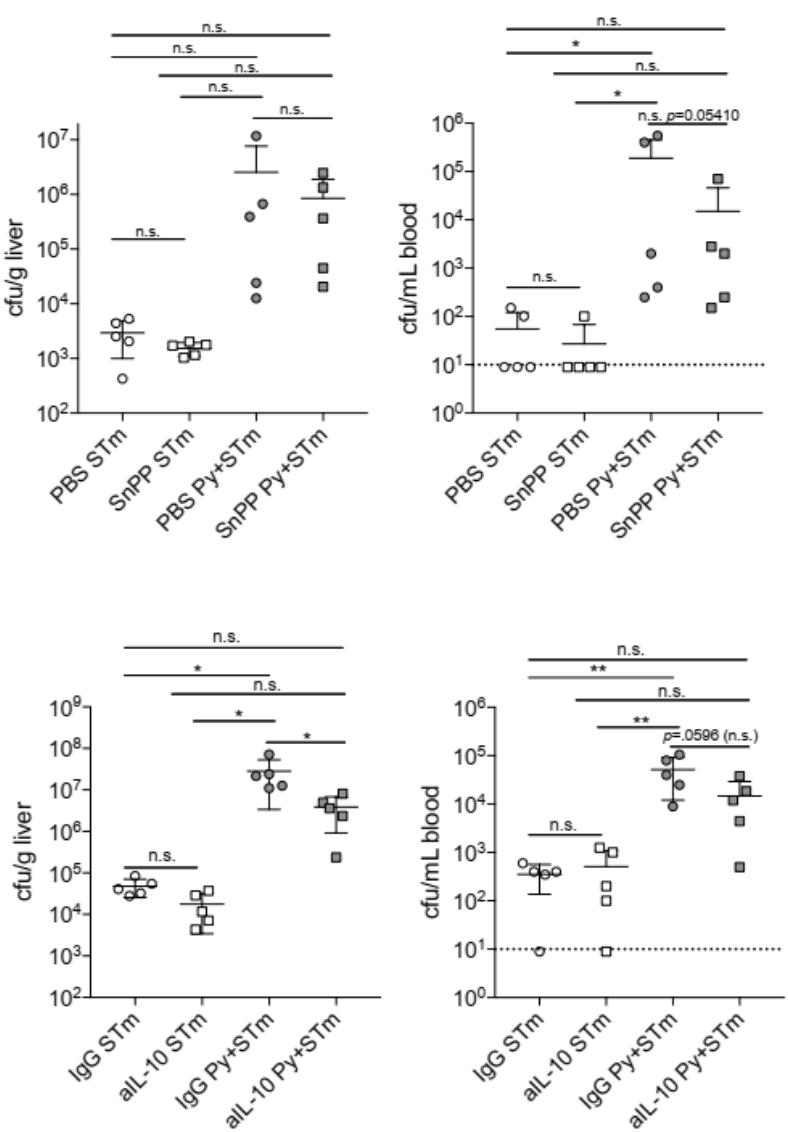

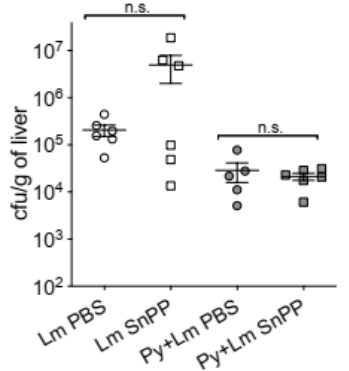

D

Figure 7. Py induction of HO-1 and IL-10 impair immunity to NTS but not Lm C57BL/6N mice were infected with $10^{5}$ Py pRBCs, ten days later both control and Py-infected mice were infected via intraperitoneal injection with $10^{3} \mathrm{CFU}$ Salmonella enterica serovar Typhimurium (NTS). Spleen, liver, and peripheral blood bacterial burdens were determined day 2-post NTS infection. Mice were treated with tin protoporphyrin IX (Sn PP) or PBS control at 48, 24 and 8 hours before NTS infection and 18 hours postNTS infection (A), or anti-IL-10 antibody or IgG isotype control on days 9,10 , and 11 post Py (B). Data (mean \pm S.D.) are representative of two independent experiments with 5 mice per group (A) or are from a single experiment with 5 mice per group (B). C-D) C57BL/6N mice were infected with $10^{5} \mathrm{Py} \mathrm{pRBCs}$, seven days later both control and Py-infected mice were infected with 5x10 $10^{6} \mathrm{CFU} \mathrm{Lm}$. Spleen and liver bacterial burdens were determined day 2-post Lm infection. Mice were treated with tin protoporphyrin IX (Sn PP) or PBS control at 48, 24 and 8 hours before Lm infection A), or aIL-10 antibody or isotype control on days 6, 7, and 8 post Py B). Data (mean \pm S.E.) are cumulative results from two independent experiments with 3 to 6 mice per group analyzed with one-way ANOVA followed by Tukey's multiple comparisons test. 


\section{Plasmodium yoelii infection suppresses innate immunity to Streptococcus pneumoniae}

(Sp) is one of the most common bacteria observed in individuals co-infected with malaria and an invasive bacterial infection $[42,45]$. To formally test the ability of Plasmodium to impair innate immunity to Sp, mice were infected with Py followed by intranasal Sp infection 7-days later. Following infection, diplococci were clearly evident in the blood of co-infected mice (Fig. 8A), which showed lower survival through 84hours post Sp infection compared to control Sp-only infected mice (Fig. 8B). Surprisingly, there was no difference in Sp burdens 3-days post-infection in the lungs of Py-infected and control mice (Fig. 8C), suggesting that Py infection does not impair colonization of Sp in the lung. In contrast to the lung, and consistent with the decreased survival of Py-infected mice following Sp infection, there were substantial increases in Sp burden in both the blood (Fig. 8D) and spleen (Fig. 8E). These data demonstrate that Py infection also impairs the innate immune response to Sp bacteremia. 

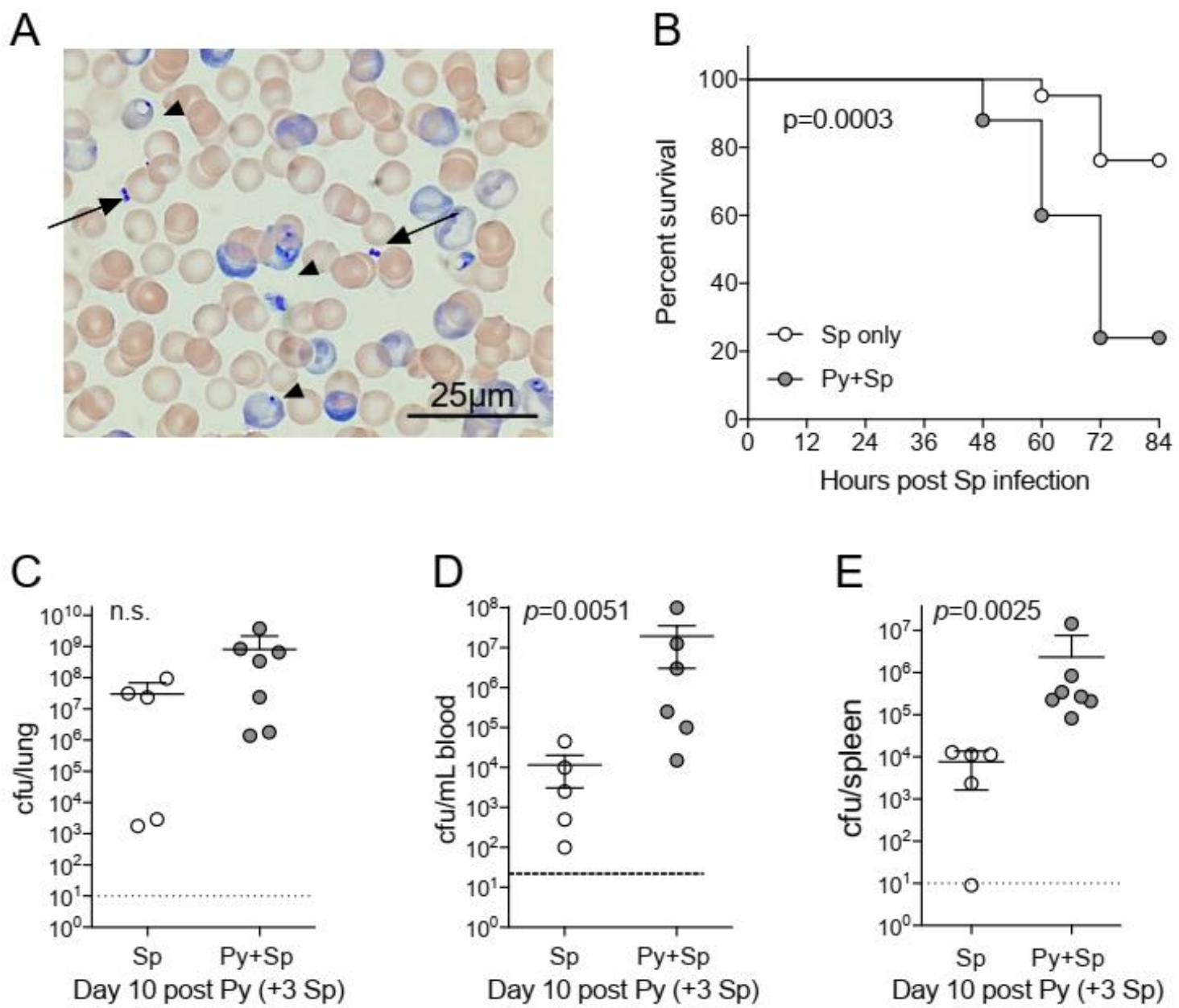

Figure 8. P. yoelii infection increases severity of Pneumococcal infection. C57BL/6N mice were infected (i.v.) with $10^{5} P$. yoelii infected RBCs, 7-days later Py-infected and naïve mice were infected (i.n.) with $10^{6} \mathrm{CFU}$ Streptococcus pneumoniae (Sp). A) Giemsa stained blood smear from co-infected mouse. Arrows indicate Sp diplococci, arrow heads indicate $P$. yoelii infected RBCs. B) Survival of mice following Sp infection. Data are pooled results from 4 independent experiments with a total of $21 \mathrm{Sp}$ only mice and $25 \mathrm{Py}+\mathrm{Sp}$ co-infected mice. Survival data was analyzed by log-rank (Mantel-Cox) test. C-E) Mice were sacrificed at either day 3 post Sp infection (10 days post Py infection) or when mice became moribund. Sp burden was quantified in lungs (C), blood (D), and spleen (E). (C-E) Data (mean \pm S.D.) are from a single experiment that is representative of 3 independent experiments with 5 to 7 mice per group. 


\section{Py infection induces long-term impaired immunity to Sp infection.}

Similar to the long-term defect in innate immunity to Lm in mice following clearance of Py infection (Fig. 4C), mice infected with Sp, both 30 days-post Py infection (Fig. 9A-C) or 65-72 days-post Py infection (Fig. 9D-F), had increased bacterial burdens two days-post Sp infection in lungs, peripheral blood, and spleens compared to age matched mice infected with only Sp. These data demonstrate that Py infection also impairs the innate immune response to Sp bacteremia, and that the Plasmodium-driven susceptibility to $\mathrm{Sp}$ is prevalent beyond the duration of the parasite infection.
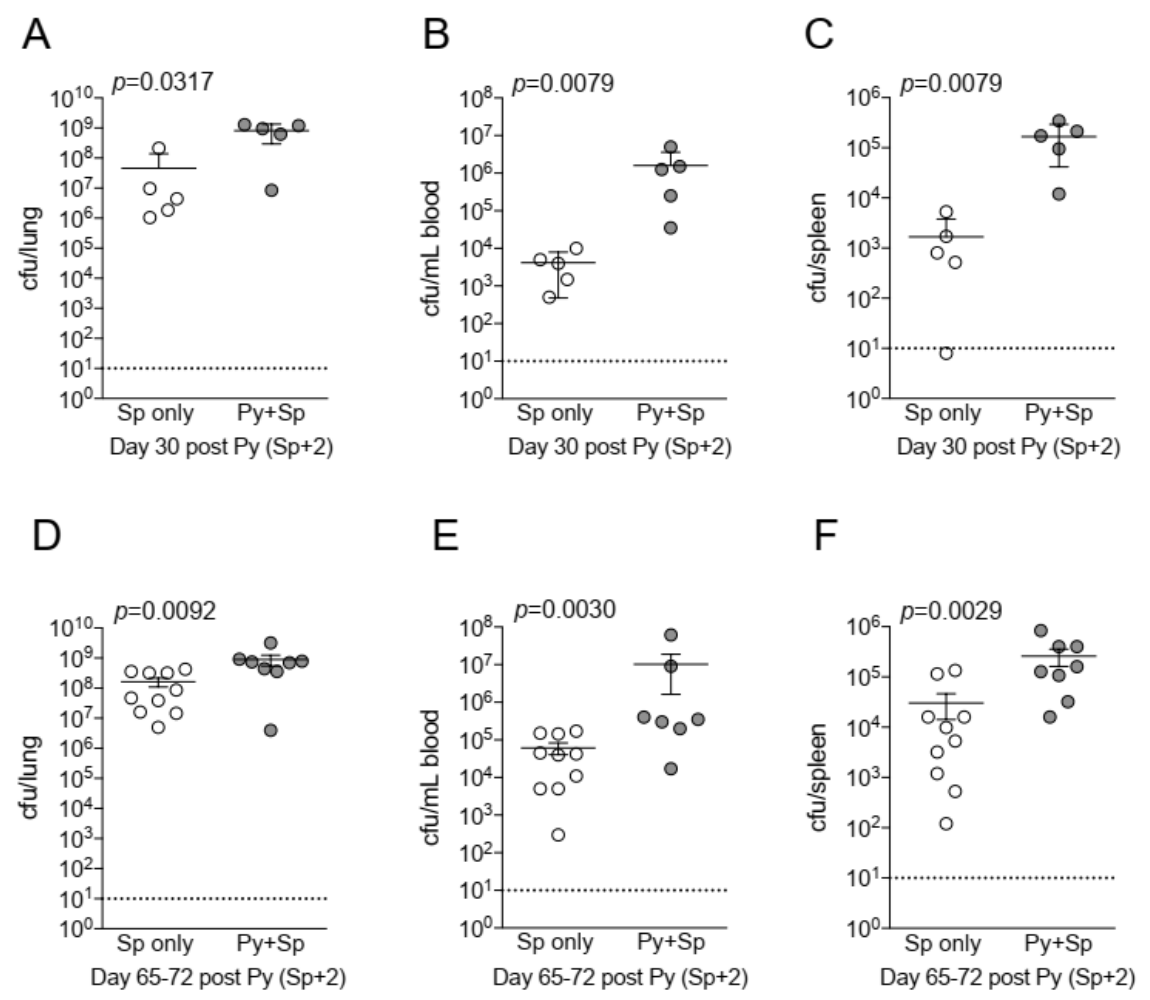

Figure 9. Py infection induces long-term impaired immunity to $\mathrm{Sp}$ infection. C57BL/6N mice were infected with $10^{5} \mathrm{P}$. yoelii infected RBCs, then infected with $10^{6} \mathrm{CFU}$ Sp either 30 (F-H) or 65-72 (I-K) days later. Sp bacterial burdens in blood and organs were obtained from mice sacrificed 2 days after $\mathrm{Sp}$ infection. (F-H) Data (mean \pm S.D.) representative of 2 independent experiments with 5 to 7 mice per group. (I-K) Data (meat \pm S.E.) cumulative results from 2 independent experiments with 7 to 10 mice per group. (C-K) Dotted lines indicate limit of detection. Each symbol represents a single mouse. Statistical significance was determined with Mann-Whitney test. 
P. yoelii-induced IL-10 contributes to impaired resistance to Pneumococcal coinfection

Since HO-1 and IL-10 have been found to impair innate immune responses in the context of malaria coinfections with NTS, we explored the possibility that these factors also contribute to the impaired resistance to Sp that we have observed in our Py coinfection model system. Contrary to observations in NTS coinfections, Sn PP treatment did not improve anti-bacterial immunity in Py+Sp co-infected mice (Fig. 10A), as bacterial burdens of Sn PP treated mice were comparable to control treated groups. Blocking IL-10 with an anti-IL-10 monoclonal antibody treatment prior to Lm infection did not improve bacterial clearance in co-infected mice (Fig. 7B). Similar to NTS (Fig. 7B) while in contrast to LM (Fig. 7D), anti-IL-10 monoclonal antibody treatment in Py+Sp co-infected mice was sufficient to significantly reduce the severity of Pneumococcal infection compared to control IgG treated Py+Sp mice (Fig. 10B). These data demonstrate that Plasmodium-induced IL-10 is part of the mechanism by which Py infection impairs control of Sp. The data from our explorations of both the Py+Lm and $\mathrm{Py}+\mathrm{Sp}$ coinfection model systems indicate that there may be additional mechanisms by which Plasmodium infections impair control of systemic bacterial infections. 

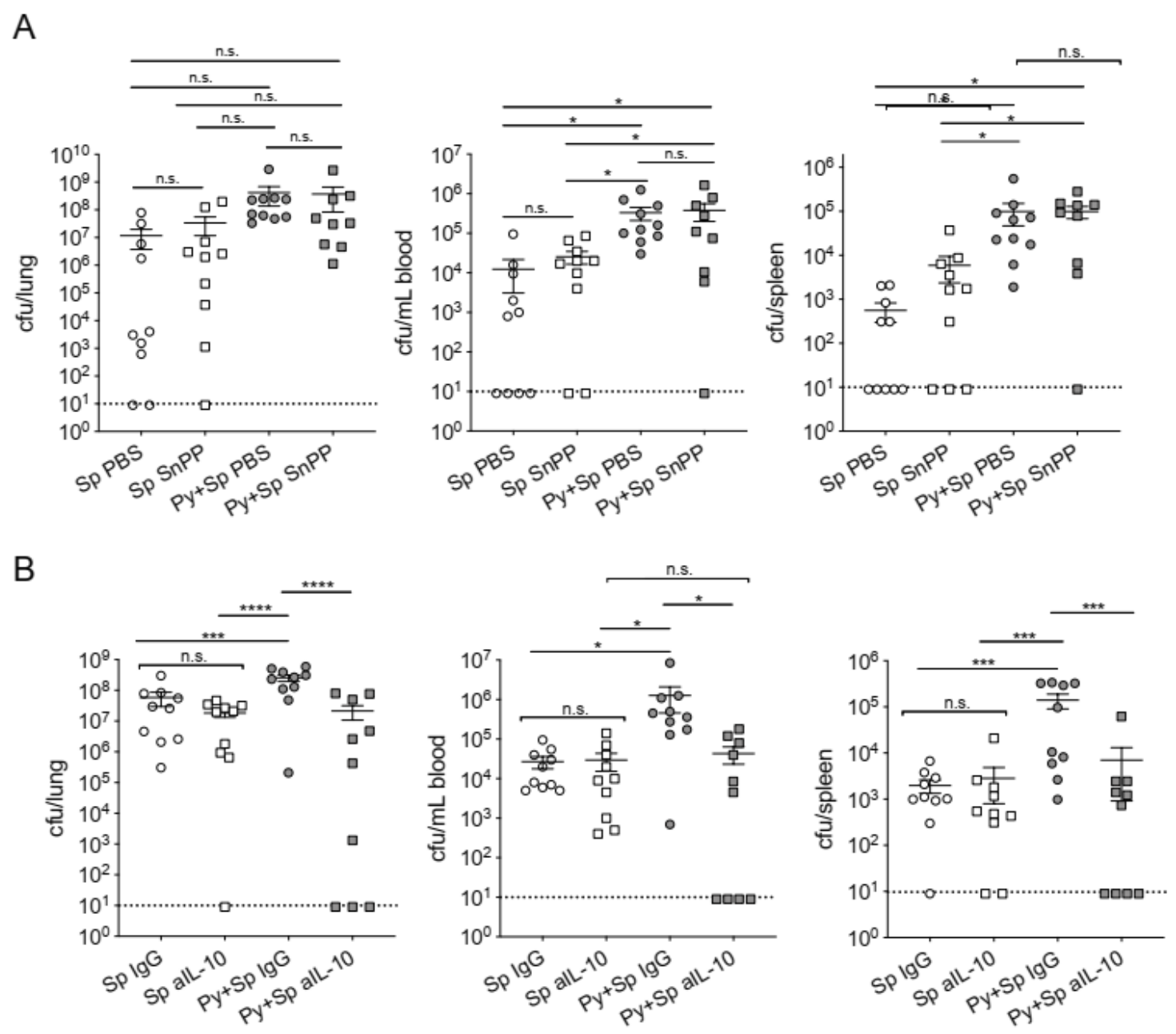

Figure 10. IL-10 derived from Py infection impairs immunity to Sp. C57BL/6N mice were infected with $10^{5}$ Py pRBCs, eight days later both control and Py-infected mice were infected i.n. with $5 \times 10^{5} \mathrm{CFU}$ $\mathrm{Sp}$. Lung, spleen, and peripheral blood bacterial burdens were determined day 2-post Sp infection. Mice were treated with tin protoporphyrin IX (Sn PP) or PBS control at 48, 24 and 8 hours before Sp infection and 24 hours post Sp infection A), or $\alpha$ IL-10 antibody or isotype control on days 6, 7, and 8 post Py B). Data (mean \pm S.E.) are cumulative results from two independent experiments with 5 mice per group and analyzed with one-way ANOVA followed by an uncorrected Fischer's LSD test with multiple comparisons, n.s., not significant. 


\section{$\underline{\text { Discussion }}$}

Complications associated with bacterial coinfections during malaria have been the subject of numerous clinical reports. Several basic scientific studies have reported mechanisms by which Plasmodium infections impact susceptibility to invasive bacterial infections, but these have exclusively focused on suppressed immunity to NTS. A broad range of bacterial pathogens are isolated from bacteremic patients with malaria, yet, other than what is known about NTS coinfections, very little is known about the mechanisms that cause increased risk for invasive bacterial diseases during malaria. Identifying specific or more general deficiencies in innate immune responses during Plasmodium infections that are responsible for diminished bacterial immunity is necessary to improve the treatment of these cases. In this project, we sought to create tools that could be used to explore the underlying mechanisms of malaria-induced immune suppression and investigate what factors contribute to impaired innate immunity to invasive bacterial infection during acute Plasmodium infection and during convalescence. Prior reports on NTS infections during malaria, demonstrated that infection-induced IL-10 production and infection-induced hemolysis, and activation of $\mathrm{HO}-1$, were central to impaired immunity to NTS. Here, we have demonstrated that Plasmodium infections also impair innate immunity to bacterial infections independent of HO-1 and IL-10. By establishing coinfection model systems with Lm and Sp, we have found that this defect in immunity to secondary bacterial infection extends beyond clearance of the parasite and that the impaired immunity to $\mathrm{Sp}$ is partly due to the immune suppressive activity of Py induced IL-10. 
It has been postulated that Plasmodium-induced immune suppression and susceptibility to invasive bacterial infections is not attributed to a parasite-specific factor, but rather is due to Plasmodium-induced hemolysis, and subsequent activation of HO-1 [109]. Additionally, Cunnington and colleagues demonstrated that neutrophils collected from patients previously infected with Plasmodium falciparum had defective ROS burst following PMA stimulation that lasted up to 8 weeks-post treatment [61]. Another recent clinical investigation found decreased cellular- and serum- mediated bacterial(NTS) capacity in Malawian children with current or convalescent P. falciparum malaria [64]. This long-term Plasmodium-driven defect in NTS immunity was attributed to impaired opsonophagocytic activity and contributed to decreased PMN production of reactive oxygen species (ROS) following stimulation with serum-opsonized NTS. We demonstrate that Py-induced immune suppression lasts up to 2-months post clearance of Py, when the effects of hemolysis are likely no longer in play. Of note, tissue deposition of hemozoin byproducts from $P$. berghei infection remains deposited in liver, bone marrow, and spleen tissues as well as within isolated $\mathrm{CD} 11 \mathrm{~b}^{+}$cells isolated from those tissues and peripheral blood of mice for at least 196 days post infection [110]. The contribution of hemozoin as a factor involved in Plasmodium-dependent suppression of anti-bacterial immune functions will be revisited in Chapter IV.

In our examination of the impact of Plasmodium infection on production of an inflammatory cytokine, IFN $\gamma$, we observed that the capacity of IFN $\gamma$ production in response to Lm infection was dependent on the amount of time post Py infection that mice were infected with Lm (Fig. 6A and B). Py+Lm coinfected mice responded with lower production when Lm infection began 7 days post Py infection compared to mice 
only receiving Lm. Surprisingly, the opposite was true when the Lm infection occurred 100 days post Py infection, as we found higher levels of IFN $\gamma$ in serum and spleens of coinfected mice. The data in this set of experiments may suggest that Py infection may induce a state of hyperresponsive "trained immunity", where the Plasmodium infection induces an innate immune "memory" that increases responses to a secondary infection [111]. The concept of innate immune memory refers to a hypo- or hyperresponsive state that occurs in innate immune cells where they are primed by an initial stimulus to respond in an extreme manner [111]. Several previous studies have demonstrated data that support the idea of trained immunity in malaria. Schrum et al. showed that in vitro treatment of healthy human PBMCs with synthetic hemozoin or P. falciparum infected RBCs, and also cells from Kenyan children with febrile malaria, were hyperresponsive in response to TLR2 stimulation in an IFN $\gamma$ independent manner [112]. Another study describes hyperresponsive of human PBMCs and murine splenocytes from P. falciparum or $P$. chabaudi infected individuals, respectively, that was dependent on TLR9, MyD88, and to a greater extent IFN $\gamma$-signaling [113].

The hyperresponsiveness of innate immune cells in the context of malaria has also been attributed to the increased susceptibility to secondary bacterial infections. One study tested responses of PBMCs from febrile malaria patients and observed that malariaprimed cells produced pathological amounts of IL-1 $\beta$ following stimulation with LPS, modeling a secondary bacterial "superinfection" [114]. In this study IFN $\gamma$ was required for inflammasome activation and IL-1 $\beta$ production in $P$. chabaudi infected mice and treatment of these mice with IL-1 receptor agonist (IL1RA) improved their survival following intragastric infection with NTS. Given the range of results in studies that focus 
on the immunomodulatory role of IFN $\gamma$ in malaria, it is unclear whether this or other cytokines are dispensable for the induction of trained immunity [112]. While hyper responses were seen in studies with $P$. chabaudi + NTS coinfection and $P$. chabaudi + LPS challenge where observations were made 7 days post Plasmodium infection, our challenge of Py-infected mice with Lm 7 days post Py did not have a greater IFN $\gamma$ response to $\mathrm{Lm}$ than mice infected with $\mathrm{Lm}$ alone. Alternatively, when the $\mathrm{Lm}$ infection began 100 days post Py infection in our Py+Lm coinfection model, we did see that the innate immune response of coinfected mice was primed to respond with a greater level of IFN $\gamma$ than Lm only mice. Our data therefore suggests that Plasmodium infection induces trained innate immunity (at least) after the initial acute phase of the blood stage of malaria, and that this affect lasts long (up to 2 months) after clearance of the parasite. By blocking the activity of IFN $\gamma$ with an anti-IFN $\gamma$ antibody and comparing bacterial burdens in spleens of Lm infected treated and untreated mice to Py+Lm coinfected mice, we were able to determine whether the impaired IFN $\gamma$ response following Lm infection that we observed in Py-infected mice during the early stage of parasitemia (7 days post Py) contributed to their delayed clearance of $\mathrm{Lm}$ from spleens (Fig. 6C). In another experiment recombinant IFN $\gamma$ was given to coinfected mice to test whether providing exogenous IFN $\gamma$ would improve their ability to clear $\mathrm{Lm}$. We found that neither the intervention of blocking IFN $\gamma$ activity in Lm only mice nor the addition of recombinant IFN $\gamma$ to coinfected mice changed their abilities to clear Lm from spleens as compared to their respective control groups. Hence, during the early blood stage of malaria the impaired IFN $\gamma$ production in response to a secondary bacterial infection does not appear to play a role in the suppressed antibacterial innate immunity in this model. 
This finding is in agreement with our data showing a decreased IFN $\gamma$ response to $\mathrm{Lm}$ infection early in Py infection but heightened IFN $\gamma$ long after Py clearance. If IFN $\gamma$ were involved in the delayed clearance of Lm in coinfected mice, we would expect IFN $\gamma$ responses to be similar at both timepoints post Py infection. Additional experiments are required to understand the underlying mechanisms and kinetics of both the impaired production of proinflammatory cytokines during Plasmodium infections as well as the hyperresponsive, "trained" innate immunity that occurs in the time following clearance of the parasite. There is also strong rationale to examine how repeated Plasmodium infections in individuals over time may affect these occurrences, and whether they have a detrimental impact on host immunity to other bacterial pathogens, such as NTS and Sp.

Clinical reports of invasive bacterial disease coinciding with malaria often report both NTS and Sp as the two organisms most commonly isolated from blood. Experimental studies to date have focused almost exclusively on NTS and it is unknown whether the mechanisms of Plasmodium-induced immune suppression to NTS will be important for Sp or other bacterial pathogens found in coinfections with malaria (e.g., Haemophilus influenzae, Mycobacterium tuberculosis, Staphylococcus aureus, Streptococcus pyogenes, etc. $[42-49,54])$. We report for the first time, to our knowledge, a murine Plasmodium and pneumococcal coinfection. Mice were infected with Plasmodium yoelii 17XNL followed by a Streptococcus pneumoniae ATCC \#6303, a type 3 capsule serotype strain, via the natural intranasal route. Consistent with the risk of invasive Sp infections in humans with malaria [42], we found decreased survival and increased bacterial burdens in co-infected mice compared to control Sp only infected mice (Figure 8). Further work is required to achieve a better understanding of the factors 
at play in this coinfection. Future efforts should examine different serotypes of Sp, as the pathogenicity of $\mathrm{Sp}$ is highly variable in many disease models and to suggest that ATCC strain \#6303, or even that type 3 capsule Sp represent all Sp would be an overstatement [115].

The impact of hemolysis-related immune suppression initiated by Plasmodium infection (activation of HO-1 and IL-10) has been well studied in the context of NTS in both animal models and clinical studies $[59,61,109]$. By exploring coinfection models with different bacterial pathogens during malaria, we sought to determine whether the IL10-dependent and HO-1-dependent mechanisms were specific to NTS coinfection, or if they also play a role in malaria-related immunosuppression to other bacterial coinfections. While our results indicated that HO-1 inhibition with Sn PP failed to improve immunity to either Lm or Sp coinfections (Fig. 4C and Fig. 7A), we did see a reduction in Sp bacterial burdens in co-infected mice treated with anti-IL-10 (blocking) antibody (Fig. 7B). This result is in line with observations made in influenza and pneumococcal coinfections, where IL-10 production following influenza virus infection disrupts the inflammatory response to $\mathrm{Sp}$ in a manner that favors dissemination and invasive disease [116]. Blocking IL-10 activity in the Py+Sp coinfection may, similarly to what is seen in the influenza coinfection [116], results in increased levels of inflammatory factors in the respiratory tract, such as TNF $\alpha$ and IFN $\gamma$, enhanced PMN infiltration to lungs, and restore the antibacterial immune response to Sp to normal. IL-10 is known to impair neutrophil chemotaxis, which may contribute to "neutrophil paralysis" that has been characterized in samples from patients with $P$. vivax malaria [117]. Thus, the anti-IL-10 treatments may have restored neutrophil function, resulting the reduction 
in pneumococcal burden in anti-IL-10 treated co-infected mice compared to isotypetreated controls. While this result shows that IL-10 is involved in suppressing the immune response to Sp during Py infection, it likely does not explain the long-term impaired immunity to Sp we have demonstrated (Fig. 6), since levels of IL-10 decrease following parasite clearance [118].

Curiously, we found that defective immunity to Lm following Py infection occurred only with the ActA-deficient mutant strain, while wildtype Lm 10403S was cleared more efficiently in coinfected mice (Fig. 5A). Since the strain used exclusively while characterizing the model also expressed chicken ovalbumin protein (OVA) allows for measurement of Lm-OVA-specific T-cells and quantitative assessment of adaptive immune response (also used in C. White et. al [119]), an actA-deficient strain that does not express OVA was also tested in the coinfection model prior to testing wildtype Lm. In vivo clearance of this OVA-negative, actA-deficient strain was impaired in coinfected mice similarly to the OVA-expressing strain used in the majority of this study (data not shown), suggesting that differences in immune-clearance and effect of Py infection are dependent on the presence of functional actA gene expression

Based on this biological difference of actA-positive and actA-negative Lm, it is possible that factors such as opsonizing complement factors involved in opsonophagocytosis, are absent or are rendered inactive or defective by Py infection and therefore unable to function normally against actA-negative Lm that invades new cells from extracellular space [120]. This deficiency would have no negative consequence for Lm that invades new cells mainly via direct intercellular actin-propulsion into nearby cells. In this scenario, the inflammatory nature of the Py infection may help to activate 
innate immune cells to enhance their ability to kill intracellular Lm that is unable to escape via function of actA. Combined with less reliance on evading extracellular immune components (due to their limited time spent outside of cells compared to actAdeficient Lm), enhanced killing by the Py-induced inflammatory response may explain the improved wildtype Lm clearance during coinfection that is contrary to the defective clearance observed in Py+actA-deficient Lm coinfection.

The result that demonstrated enhanced clearance of wildtype Lm conferred by Py infection is interesting, highly repeatable and further exploration of the mechanisms behind Py infection-mediated protection could uncover potential therapies for invasive Lm disease. For the scope of this project, however, continuing to pursue mechanisms in Listeria biology behind the actA-dependent defective clearance in Py coinfection would be tangential and not helpful in our pursuit of understanding Plasmodium-driven immunosuppression related to impaired to invasive bacterial coinfection. For this reason, wildtype Lm is not used experimentally or investigated in the Py coinfection model for the remainder of the project.

These findings provide a translational picture of malaria-driven long-term defects in anti-bacterial innate immunity, where we have observed Py-driven impaired immunity to $\mathrm{Lm}$ and $\mathrm{Sp}$ coinfections at all time points examined, including more than two months after clearance of infected red blood cells. Furthermore, these data indicate that the immune suppression occurs independent of Py-induced hemolysis, given that the duration of hemolysis and related physiology during malaria is limited to the active blood stage of the infection and effects of hemolysis return to normal relatively quickly. For instance, 
HO-1 activity significantly decreases in Py infected mice five days after antimalarial treatment and is comparable to uninfected mice ten days post treatment [121]. 


\section{CHAPTER III}

\section{IMPACT OF PLASMODIUM YOELII INFECTION ON SPLENIC MYELOID CELL POPULATIONS AND THEIR ANTI-BACTERIAL EFFECTOR FUNCTIONS}

\section{$\underline{\text { Introduction }}$}

The spleen, splenic innate immune cells, and their roles in infection

Plasmodium infection induces a robust inflammatory immune response characterized by substantial production of inflammatory cytokines, the effects of which are eventually regulated by anti-inflammatory factors in order to reduce collateral damage to host tissues in the process. Several organs are damaged during Plasmodium infection, including the liver, kidneys, lungs, brain, bone marrow and spleen. There is sufficient reason to believe that pathology of the spleen during Plasmodium infection is particularly important in the context of invasive bacterial infection. Under normal conditions, the spleen is responsible for clearance of senescent and damaged erythrocytes and other debris [122]. Another critical function of the spleen is to clear pathogens that have entered the blood stream. This is achieved by strategically positioned macrophages that internalize and kill circulating pathogens. By residing in close proximity to the marginal zone and the small, open ended capillaries of the penicillar arteriole [47], marginal zone macrophages (MZM), and marginal metallophilic macrophages (MMM) function as scavenger phagocytes patrolling the marginal zone space for pathogens and 
other cell debris. MZMs can interact directly with MZ B cells while MMMs can enter the white pulp of the spleen and present antigen to DCs in the marginal zone [123, 124]. From the marginal zone, approximately $10 \%$ of circulating blood flows slowly through the perifollicular zone to the reticular meshwork of the red pulp, and here damaged erythrocytes, debris, and blood borne pathogens can also become stuck and internalized by red pulp macrophages (RPM) [38]. These specific subsets of tissue-resident macrophages are equipped with a unique set of recognition receptors that allow them to efficiently identify, uptake, and kill invading pathogens.

The role of these splenic tissue-resident macrophages during blood-borne infections is well characterized for several pathogens. A direct recognition of Sp capsule polysaccharide by SIGNR-1(CD209b), a C-type lectin receptor which is highly expressed on MZMs, is involved in uptake of Sp and has been demonstrated to be critical for protection of mice against pneumococcal septicemia [125, 126]. SIGNR-1 also interacts with components of the complement cascade to promote protection vs. pneumococcal septicemia by binding $\mathrm{Clq}$ and initiating C3-convertase, initiating an Ig-independent activation of the classical complement pathway [127]. MZMs also express a scavenger receptor, MARCO, which is known to recognize gram negative bacteria and works in concert with toll-like receptor signaling to facilitate cell activation and enhanced phagocytosis functions $[128,129]$. MARCO, unlike SIGNR-1, recognizes unopsonized pathogens [130]. Specific functions of MMMs during blood-borne infections have also been described, including early control of Lm infection independent of T- and B-cell interaction in a bacteremia model, antigen presentation and indirect activation of $\mathrm{CD}^{+} \mathrm{T}$ cells, and recognition and phagocytosis of blood-borne pathogens involving MOMA-1, a 
surface molecule enriched on spleen MMMs [131, 132]. Like MZMs, RPMs have the ability to eliminate $\mathrm{Sp}$ in vivo [133]. This anti-pneumococcal ability of RPMs, however, was demonstrated in mice that were previously infected with Leishmania donovani where RPMs become more important in capture of blood-borne pathogens due to the Leishmania donovani-induced loss of MZMs. A similar phenomenon occurs in Plasmodium chabaudi infection, where both MZMs and MMMs are nearly completely lost at the height of parasitemia [134]. To what degree these splenic macrophage populations are loss following Py infection and the effect that potential loss may have on control of bacterial coinfections are not known.

One of the key indicators of a Plasmodium infection, in addition to measurable fever, is splenomegaly that is often diagnosed by clinicians when the spleen is palpable at least $10 \mathrm{~cm}$ below costal margin of the patients thoracic cage [135]. Splenomegaly during malaria, also referred to as hyper-reactive malarial splenomegaly syndrome (HMS), is the result of constant stimulation with parasite antigen during blood stage of malaria . Plasmodium-specific antibodies, especially IgM, aggregate in the form of immune complexes to drive HMS pathology [136]. Other byproducts of the heavy parasite burden in the spleen includes deposition of large amounts of malarial pigment (hemozoin), and destruction of splenic architecture that is important in maintaining normal physiological functions and innate and adaptive immune functions in context of infections $[123,134,137]$. Since the spleen is the primary organ involved in filtration of blood and cleanup and turnover of erythrocytes and cellular or foreign debris (i.e. bloodborne microbes), the spleen and the specialized phagocytic cells have several critical and direct roles in controlling the blood stage of Plasmodium infections. For this reason, 
spleen/splenic macrophages during Plasmodium infection has been the subject of many previous studies. During blood stage malaria parasitized and damaged erythrocytes, as well as other parasite and cellular debris are filtered out circulation by RPMs [39].

Based on the clear evidence that destruction and architectural disruption of the marginal zones occurs in the spleen following Plasmodium infection, it has been hypothesized that this decreases overall functional ability of the spleen to perform its role in defense against a blood-stream bacterial infection $[47,134,137,138]$. Splenomegaly and loss of both marginal zone macrophages (MZMs) and metallophilic macrophages (MMMs) occur in spleens of malaria patients who have increased risk to invasive bacterial infections [47]. Subsets of splenic macrophages serve as sentinels and provide clearance of blood-borne bacterial infections [39]. The importance of these cell types has been well documented in connection to resistance to blood-stream infections with Streptococcus pneumoniae, Listeria monocytogenes, Escherichia coli, and Salmonella typhimurium $[125,139]$. Experiments utilizing low dose clodronate liposome treatments do deplete MMMs and MZMs have further demonstrated the importance of these cells based on the impaired survival of clodronate treated mice compared to untreated control mice following infection with sub-lethal Lm infection [131]. The observation that MMMs and MZMs are lost during Plasmodium chabaudi infection led us to the hypothesis that a similar loss or decrease in these cells in the spleen following Py infection may be contributing to the impaired clearance of Lm and increased susceptibility of Sp we have described in coinfection models in Chapter II [134]. This Chapter will explore this hypothesis. 
In addition to tissue resident macrophages in the spleen, innate immune cells that are recruited from circulation also play essential roles in controlling bacteremia in both Lm and Sp infections [140-142]. Considering our data showing increased Lm and Sp burdens in spleens of Py-coinfected mice compared to control mice receiving only bacteria, we also examined the possibility that Plasmodium infection changes numbers and proportions of these cells over the course of Py infection. Robust inflammatory responses at the onset of the erythrocytic stage of Plasmodium infection activate the recruitment of neutrophils and "inflammatory" monocytes into circulation from the bone marrow where they contribute to the host control of parasite burden and induction of inflammation through production of cytokines [36, 143]. Clinical reports have found that increasing numbers of circulating neutrophils in infected individuals is positively correlated with parasite burden [144]. While monocytes are known to migrate to tissue sites of parasite sequestration [145], neutrophils do not seem to infiltrate such tissues despite systemic increases in chemokines [146]. The general lack of neutrophils in parasite loaded tissues is thought to be due to parasite-induced decreases in surface expression of CXCR2 or an increase in free heme and activity of heme oxygenase-1 [61, $117,146]$. Both monocytes and neutrophils uptake and kill pRBCs by recognizing opsonins, such as complement factors or antibody-bound pRBCs [36, 47]. While parasite-specific IgG is not required for phagocytosis by monocytes, they enhance uptake and reduce risk of severe symptomatic malaria and complement is still needed in the absence of antibody $[147,148]$. Monocytes and tissue resident macrophages (especially RPMs) also recognize unopsonized pRBCs through CD36, which also facilitates phagocytosis [149]. 
There is also rational to suspect that Plasmodium infection induces faulty antimicrobial functions in monocytes and neutrophils. Although they are critical in controlling parasite burden, studies describe defective phagocytosis functions of monocytes and neutrophils in patients with current or recent Plasmodium infection [36, 64, 150]. Another observation has been made describing a decrease in cytokine production, such as TNF-alpha and IL-6, in LPS-stimulated monocytes from Malawian children with severe malaria [151].

One of the most effective mechanisms of cell-mediated clearance of both intracellular and extracellular microbial pathogens is the production of reactive oxygen species (ROS). Through formation of ROS and its radical molecular dismutations, innate immune cells are able to directly damage and kill many pathogens and release pathogen associate molecular patterns (PAMPS) that can act as a signal for activation and recruitment of nearby cells [152]. As Plasmodium infections can alter spleen monocyte populations and impair the ability of phagocytic cells to produce ROS, both of which are important in the context of controlling blood-borne bacterial infections, we hypothesized that Plasmodium-dependent loss of monocytes and defective ROS production may be responsible for the impaired anti-bacterial immunity described in Chapter II.

The complement system acts as a critical innate immune defense against microbial infections by recognizing and labeling pathogens for both direct complementmediated killing and by enhancing the antimicrobial functions of phagocytic cells via opsonophagocytosis. It is well documented that mice with dysfunctional complement factors or lacking components of the complement system have impaired immunity to bacterial infections, including Lm, Sp, and STm [57, 127, 153, 154]. Moreover, the 
complement cascade is activated during Plasmodium infection through interactions with parasitized RBCs, leading to lower levels of serum C1q and defective complement activity compared to uninfected individuals [120, 155]. These findings led us to hypothesize that Plasmodium-induced functional defect of a serum component, such as a complement factor or opsonizing antibody, may contribute to impaired immunity against secondary bacteria coinfections. This hypothesis will be considered while testing the ability of splenic phagocytes from Plasmodium infected mice to control intracellular bacterial replication in this chapter by infecting splenic phagocytes with Lm opsonized with serum collected from either naïve or Py-infected mice.

In this chapter, the total numbers of splenic myeloid cell types will be determined over the course of $P$. yoelii infection and up to 2 months after clearance of the parasite using flow cytometry. Additionally, the overall control of intracellular Lm replication and the capacity of reactive oxygen species production of splenic phagocytes from Py infected mice will be assessed using a gentamicin protection assay and a flow cytometrybased method for detection of intracellular reactive oxygen species, respectively. 


\section{$\underline{\text { Results }}$}

Analysis of splenic myeloid cell population during Plasmodium yoelii infection and beyond parasite clearance

P. chabaudi infections disrupt splenic architecture and lead to the loss of marginal zone macrophages (MZMs) and marginal metallophilic macrophages (MMMs) [134]. As the spleen plays a vital role in the clearance of bacteria in the blood via these macrophage populations $[125,131,156]$, it led to the hypothesis that Py infection results in a loss of these cell populations that contribute to impaired clearance of $\mathrm{Lm}$. To determine the impact of Py infection on splenic myeloid cell populations, mice were infected with Py and various days after infection spleens were removed for quantification of polymorphonuclear cells (PMNs), inflammatory monocytes, red pulp macrophages (RPMs), MZMs, and MMMs. Cell types definitions are shown in Figure 11 as flow cytometry layouts describing our gating strategy. As expected, the total cellularity of the spleen increased through resolution of Py infection, followed by a return to baseline at one- and two-months post clearance of Py (Fig. 12A). Analysis of PMNs revealed a fairly consistent expansion in frequency (Fig. 12B) and total number (Fig. 12C) of PMNs at days $10,30-33$, and $65-67$ post-infection. Whereas the frequency of inflammatory monocytes remained consistent between Py-infected and control mice (Fig. 12D), there was an increase in the total number of these cells at days 10 and 30-33 in Py-infected mice (Fig. 12E). The frequency of RPMs were lower in Py-infected mice at days 30-33 and 65-67 post-infection (Fig. 12F), yet the total cell numbers remained fairly consistent during acute parasitemia and following resolution of infection (Fig. 12G). Consistent with the previous report [134], we observed a decrease in the frequency (Fig. 12H) and total number (Fig. 12I) of MZMs at days 30-33, 65-67, and 101-102 post-Py infection; 
however, there was no difference in $\%$ of total splenocytes at day 10 post-Py infection. In contrast, the frequency (Fig. 12J) and total number (Fig. 12K) of MMMs remained fairly consistent following Py infection. Although we did not expect changes in abundance of eosinophils or dendritic cells to have an impact on bacterial clearance, we included these cell types in our analysis to avoid misidentifying them as any of the other cells of interest (Fig. 13). Collectively, there is no consistent increase or decrease in these cell populations that correlates with impaired clearance of Lm in Py-infected mice, suggesting that alternative mechanisms explain impaired anti-bacterial innate immunity following Py infection. 

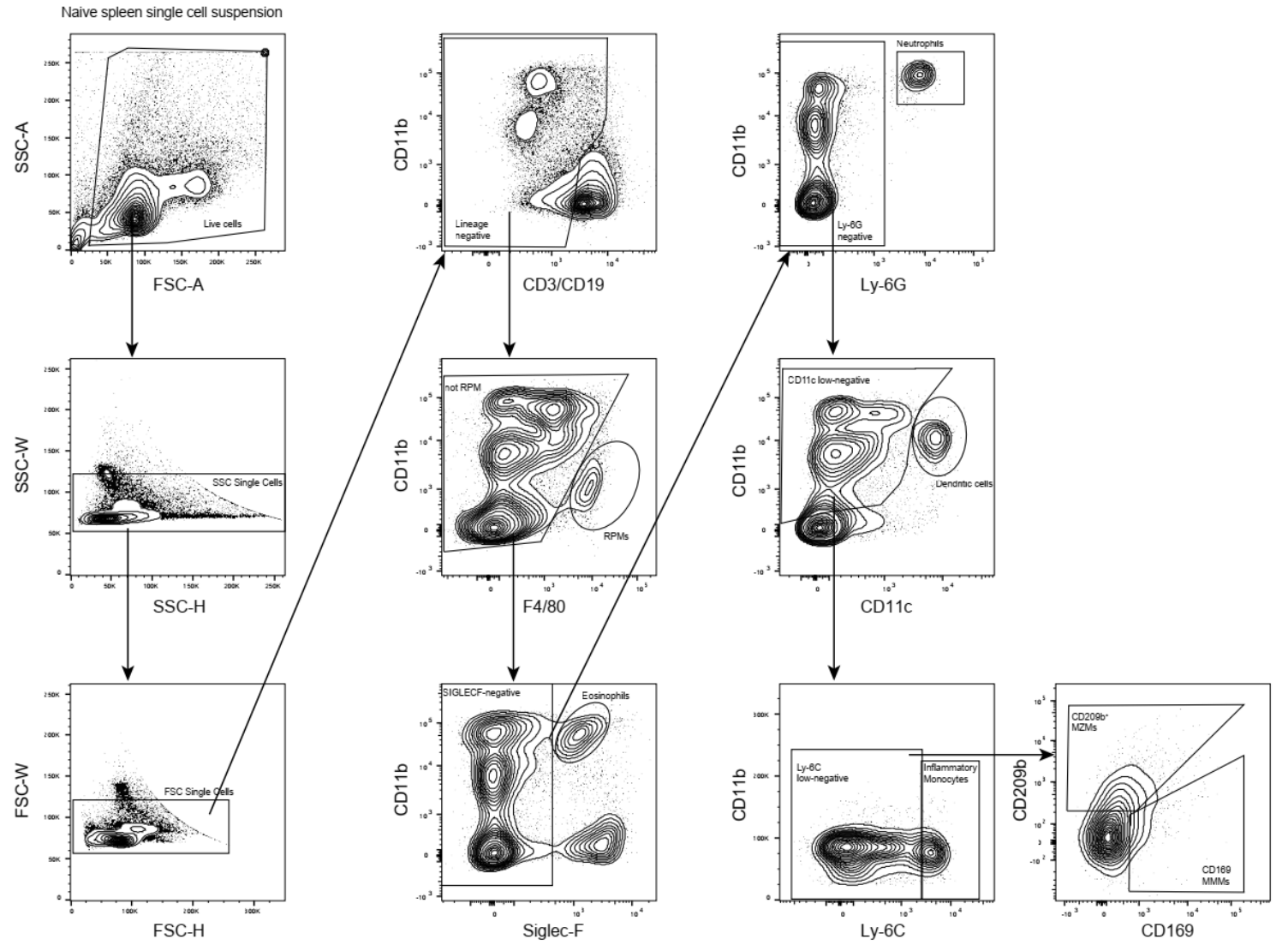

Figure 11. Gating strategy for splenic myeloid cell populations.

Single cell suspensions of spleens collected from mice infected with Py or age-matched naïve controls were prepared and stained with antibodies for flow cytometry analysis. Representative contour plots are from a naïve mouse. Arrows represent the hierarchical gating strategy that was used to determine the indicated cell populations. RPMs were gated early to avoid misidentification due to the highly auto-fluorescent nature of this cell type [157]. 

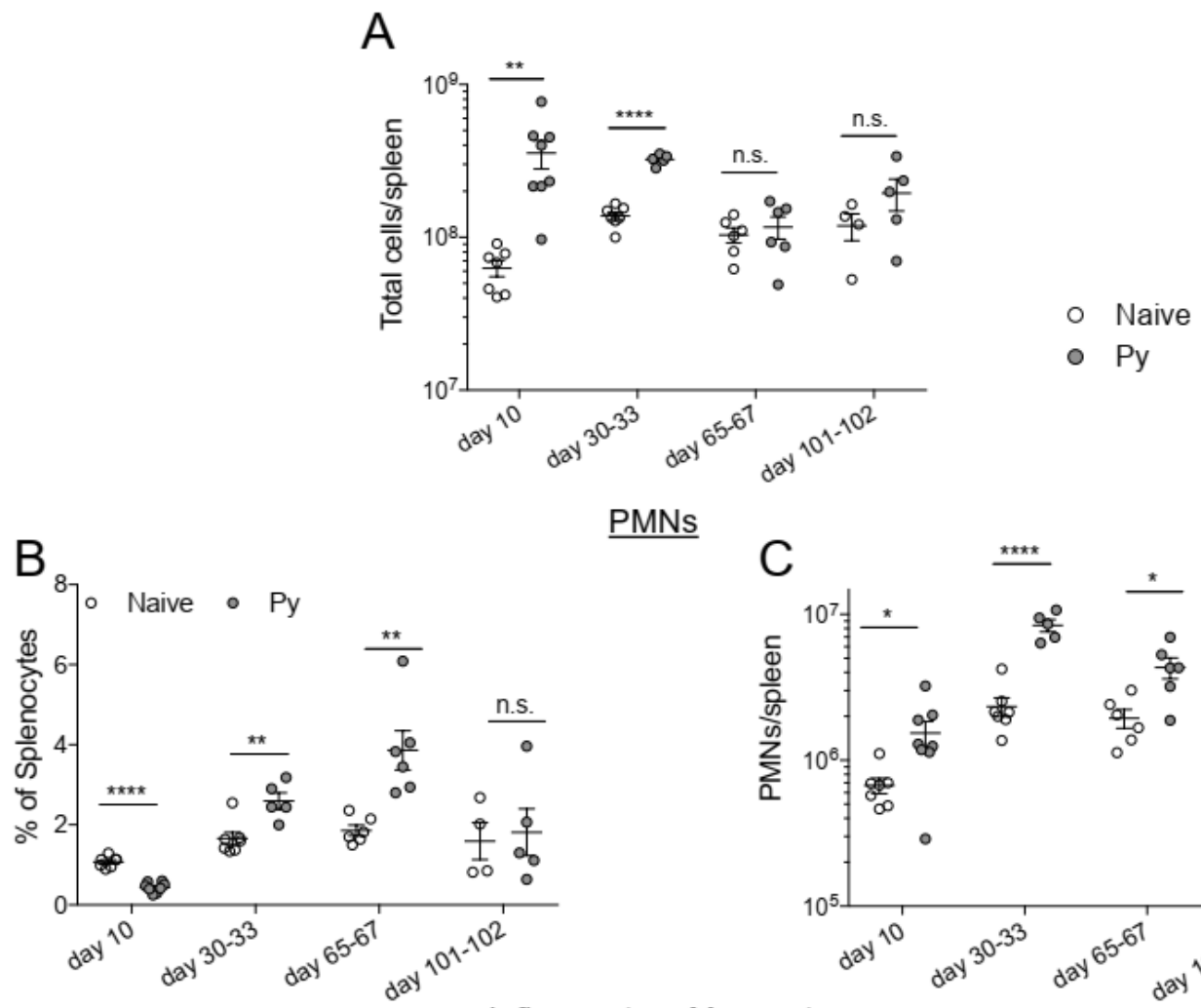

$\underline{\mathrm{PMNS}}$
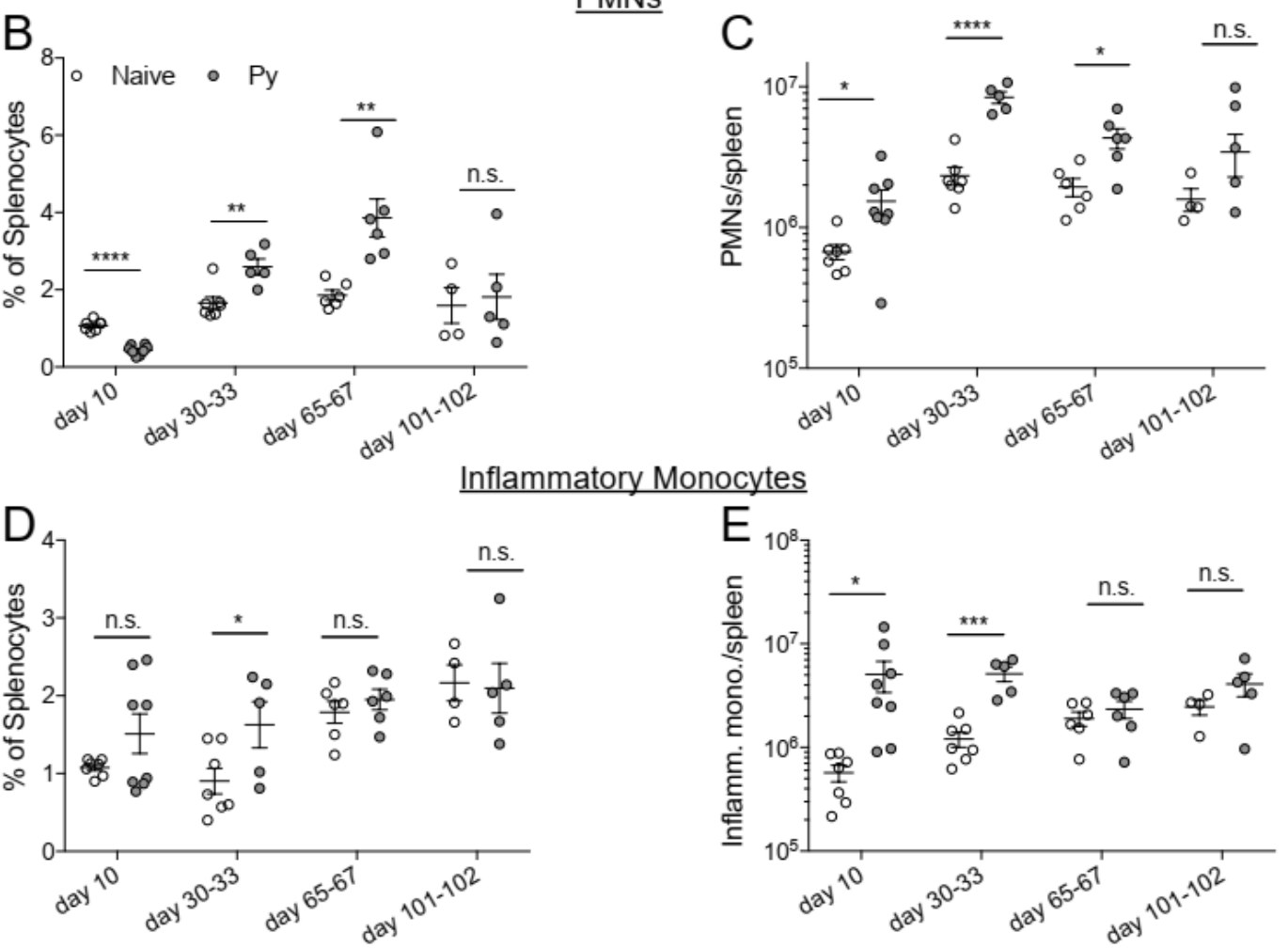

Figure 12. P. yoelii-induced temporal changes in splenic myeloid cell populations do not correlate with impaired immunity during bacteremia. C57BL/6N mice were infected with $10^{5} \mathrm{Py}$ pRBCs. Spleens were collected from control or Py-infected mice on the indicated days post infection. A) Total numbers of cells per spleen on the indicated days. Myeloid cell populations were defined as follows: PMNs (CD3CD19-, F4/80', SIGLEC', CD11b ${ }^{+}$, Ly-6G $^{+}$) (B-C), inflammatory monocytes (CD3-, CD19-, F4/80',

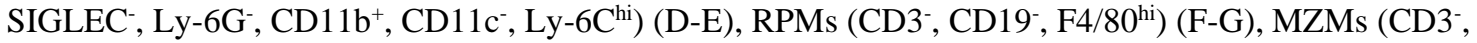

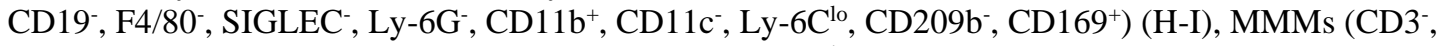

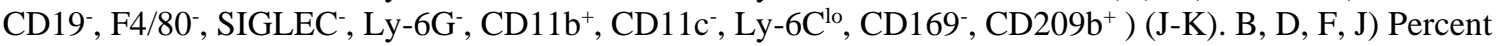
of splenocytes that are the indicated myeloid cell population. C, E, G, I, K) Total number of indicated myeloid cell population per spleen. Data (mean \pm S.E.) are combined results from $1 *$ to 3 -independent experiments per timepoint with 3-5 mice per group analyzed with $t$-tests. *Analysis of MMMs on day 10 post Py infection is a single experiment. 
Figure 12 Continued

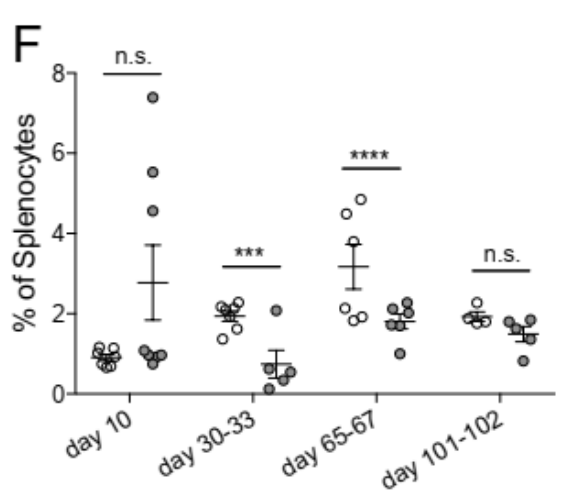

$\underline{\text { RPMs }}$

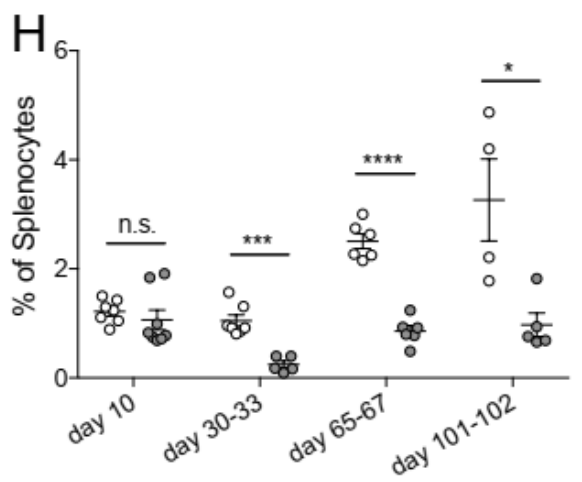

$\underline{\text { MZMs }}$
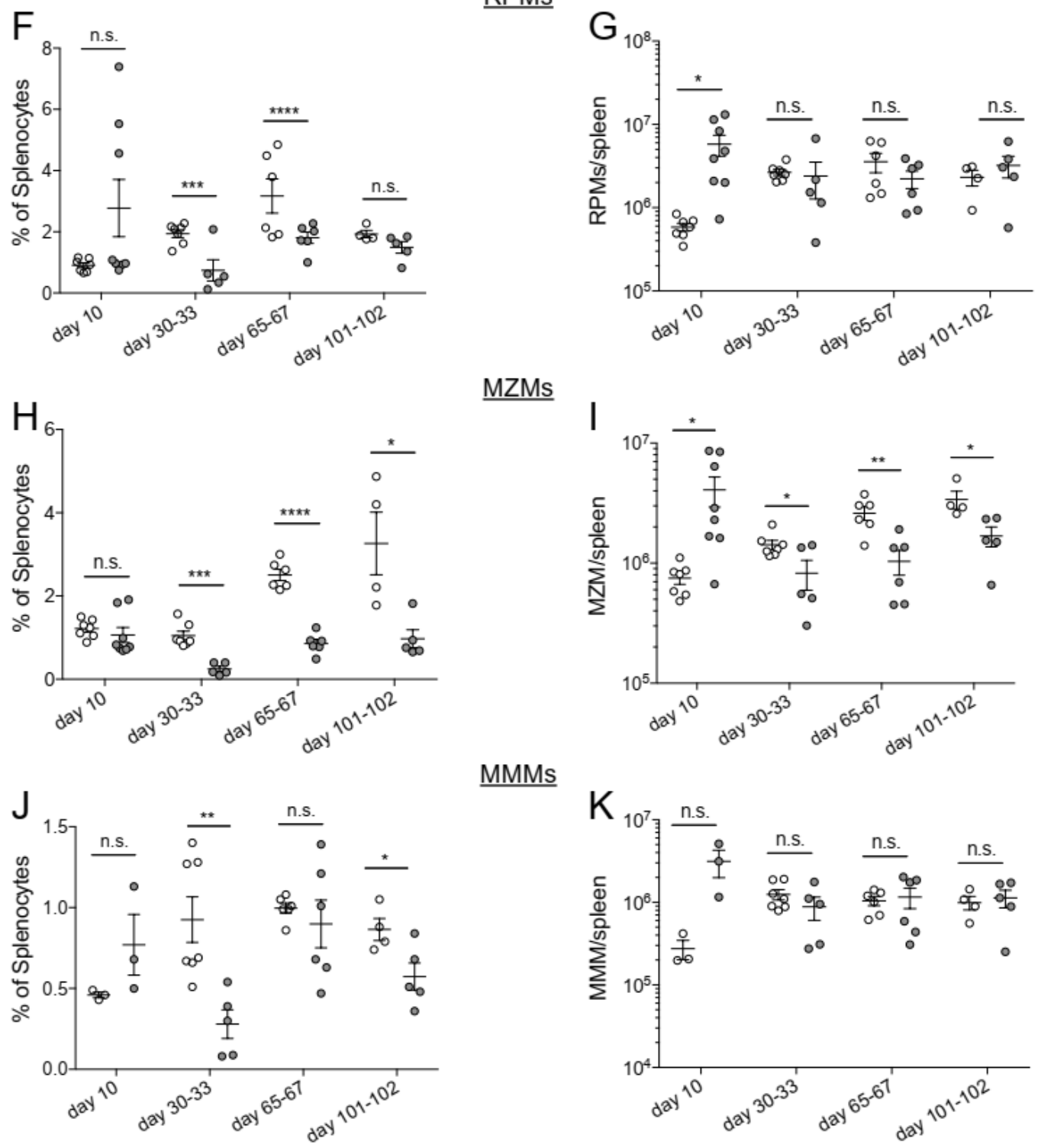

MMMs

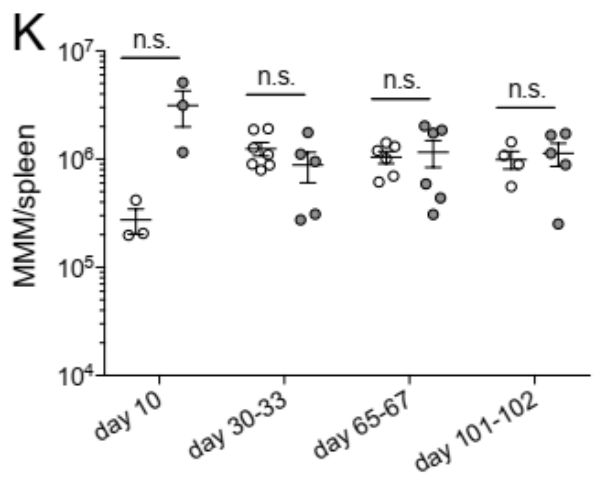

64 
A

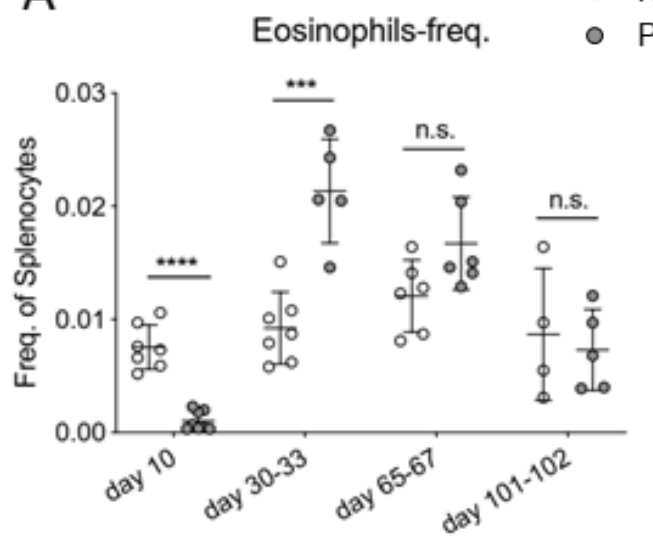

C

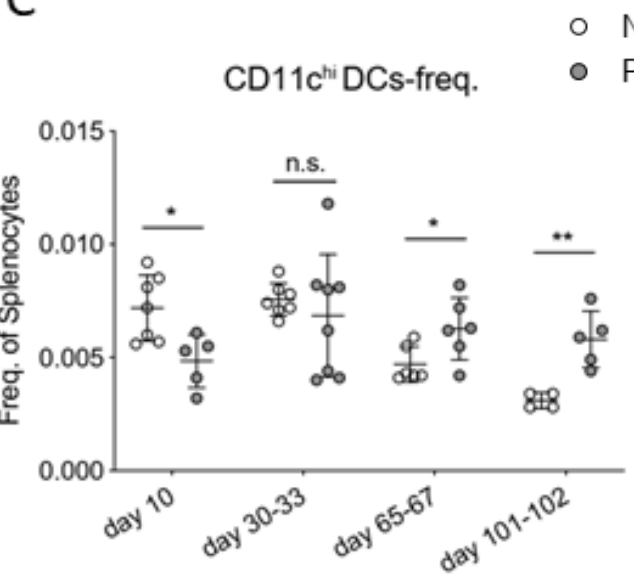

B

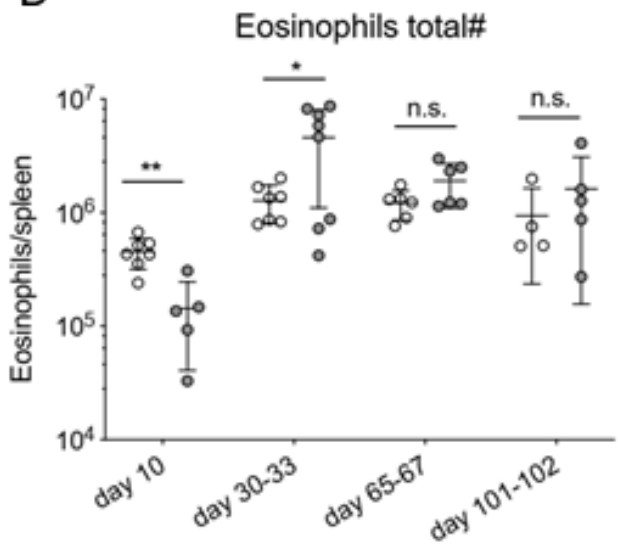

D

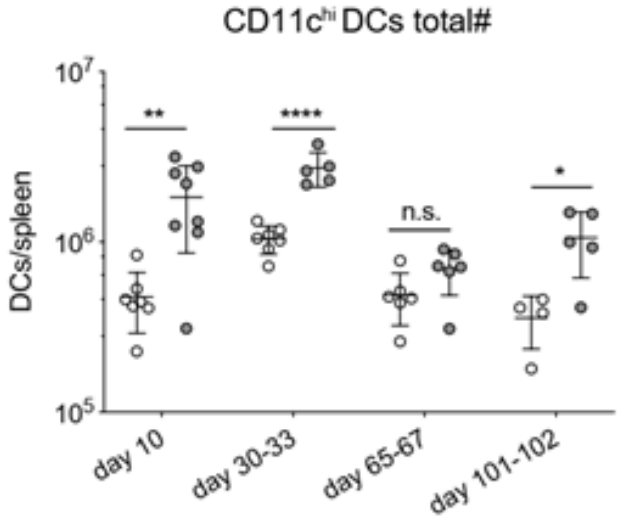

Figure 13. Analysis of Eosinophils and CD11 $c^{\text {hi }}$ Dendritic Cell populations over the course of $P$. yoelii infection. C57BL/6N mice were infected with $10^{5} \mathrm{Py}$ pRBCs. Spleens were collected from control or Py-infected mice on the indicated days post infection. A, C) Total numbers of cells per spleen on the indicated days. Myeloid cell populations were defined as follows: Eosinophils (CD3-, CD19-, F4/80',

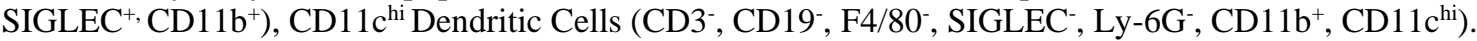
B, D) Percent of splenocytes that are the indicated myeloid cell populations . Data (mean \pm S.E.) are combined results from 3-independent experiments per timepoint with 3-5 mice per group analyzed with $t$ tests.

\section{Effect of Plasmodium yoelii Infection of Anti-bacterial Functions of Innate Immune Cells}

Data obtained from the myeloid cell analysis did not show changes that correlated

with our observation that Py+Lm coinfected mice have impaired Lm clearance up to 100 days post Py-infection (Fig. 4), suggesting that antimicrobial functions of innate immune cells may be compromised by Plasmodium infection. To determine if impaired ROS 
production is a mechanism by which Py suppresses innate immunity during bacteremia, spleens were removed from naïve control and Py-infected mice. ROS production was quantified in whole spleen single cell suspensions in untreated cells or cells stimulated with PMA, unopsonized Lm, Lm opsonized with serum from naïve mice (N-Ops Lm), or Lm opsonized with serum from Py-infected mice (Py-Ops Lm). In general ROS production was much more pronounced in $\mathrm{Ly}-6 \mathrm{G}^{+} \mathrm{CD} 11 \mathrm{~b}^{+} \mathrm{PMNs}$ than $\mathrm{Ly}-6 \mathrm{G}^{-} \mathrm{CD} 11 \mathrm{~b}^{+}$ "macrophages" (Fig. 14A). Consistent with increased Lm burden in Py-infected mice, PMNs from Py-infected mice showed decreases in intracellular ROS production (DCF geometric mean fluorescence intensity signal) in each of the stimulated groups (Fig. 14BC). In contrast, there was no difference in ROS production in $\mathrm{Ly}-6 \mathrm{G}^{-} \mathrm{CD} 11 \mathrm{~b}^{+}$cells from Py-infected and naïve mice (Fig. 14D). Similar results were also observed when splenocytes were stimulated with heat-treated (Ht) Sp, Ht N-ops Sp, or Ht Py-ops Sp (Fig. 15).

Additional analysis of the data suggests that Py infection may impede immunity to $\mathrm{Sp}$, at least in part, through a serum-dependent effect. As seen in Figure 15C, Ht Sp opsonized with serum from naive mice (Ht N-ops Sp) compared to Ht Sp opsonized with serum from Py-infected mice (Ht Py-ops Sp) elicited increased ROS production in PMNs from naïve mice (naïve cells treated with $\mathrm{Ht} \mathrm{N}-\mathrm{Ops} \mathrm{Sp}$ vs. naïve cells treated with $\mathrm{Ht} \mathrm{Py}-$ Ops Sp; open circle comparison in last two groups; unpaired $t$-test, $p=0.00087$ ). An even more pronounced serum-dependent effect was observed in PMNs from Py-infected mice (Py cells Ht N-Ops Sp vs. Py cells Ht Py-Ops Sp; gray-filled circle comparison in last two groups; unpaired $t$-test, $p=0.00042$ ). Of note, this serum-dependent effect was not 
observed following stimulation of Lm (Fig. 14C). Collectively, these results identify impairment of ROS production in neutrophils as a potential mechanism by which Py suppresses antibacterial innate immunity during systemic bacterial infections, with an additional serum-dependent factor further hindering the cellular response to $\mathrm{Sp}$. 
A

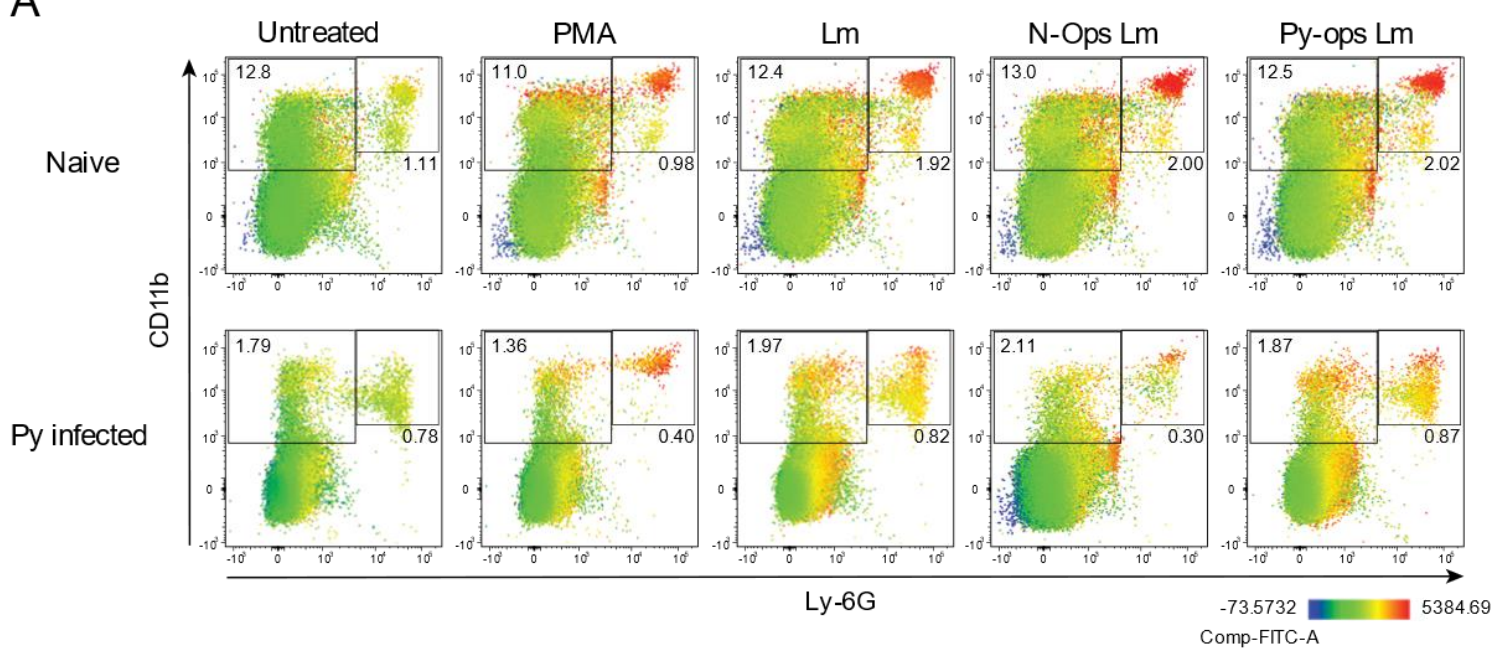

B
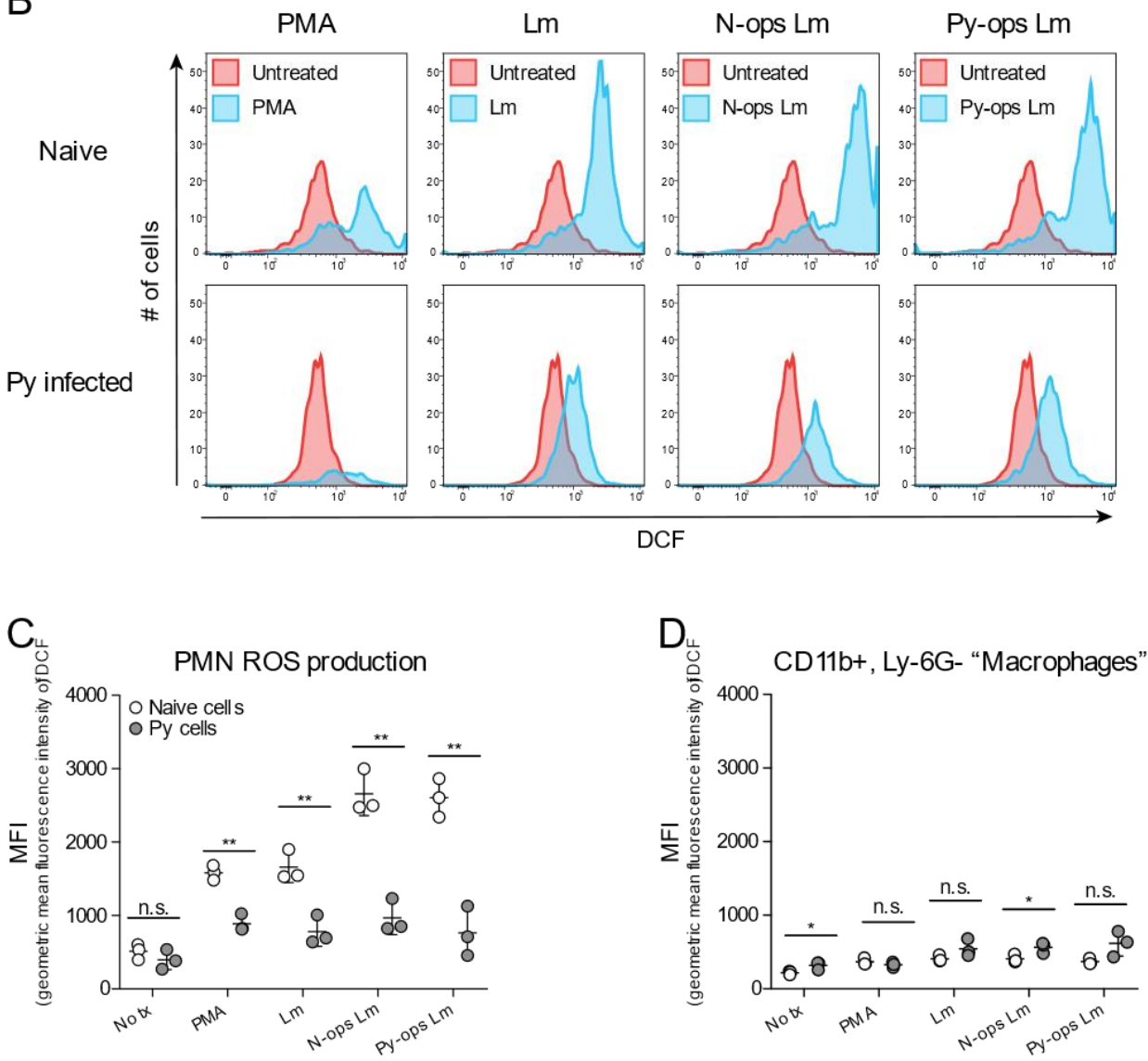

Figure 14. Splenic PMNs from $P$. yoelii infected mice have defective ROS production in response to Lm. C57BL/6N mice were infected with $10^{5}$ Py pRBCs. Single cell suspensions from whole spleens of naïve and Py-infected mice (days 14 or 15 p.i.) were incubated with DFC to detect intracellular ROS. Cells were then treated with PMA, Lm, or Lm opsonized with $10 \%$ serum from naïve or Py-infected mice. A) Heat map displays DCF fluorescence in CD11b x Ly6G cells to indicate amount of intracellular ROS. Heat maps are from an individual mouse in each group that are representative of a single experiment and of three total independent experiments. B) Histogram showing DCF fluorescence of splenic PMNs 
$\left(\mathrm{CD}_{11} \mathrm{~b}^{+} / \mathrm{Ly}_{6 \mathrm{G}}{ }^{+}\right)$as shown in box in $(\mathrm{A})$. DCF geometric mean fluorescence intensity in PMNs

$\left(\mathrm{CD}_{11} \mathrm{~b}^{+} / \mathrm{Ly}_{6 \mathrm{G}}{ }^{+}\right)(\mathrm{C})$ and macrophages/monocytes $\left(\mathrm{CD} 11 \mathrm{~b}^{+} / \mathrm{Ly}_{6 \mathrm{G}}\right)(\mathrm{D})$ populations as indicated in $(\mathrm{A})$. Each symbol represents an individual mouse. Data (mean \pm S.D.) are representative of three total independent experiments with 3 mice per group. Statistical significance was determined with multiple unpaired $t$-tests. 
A
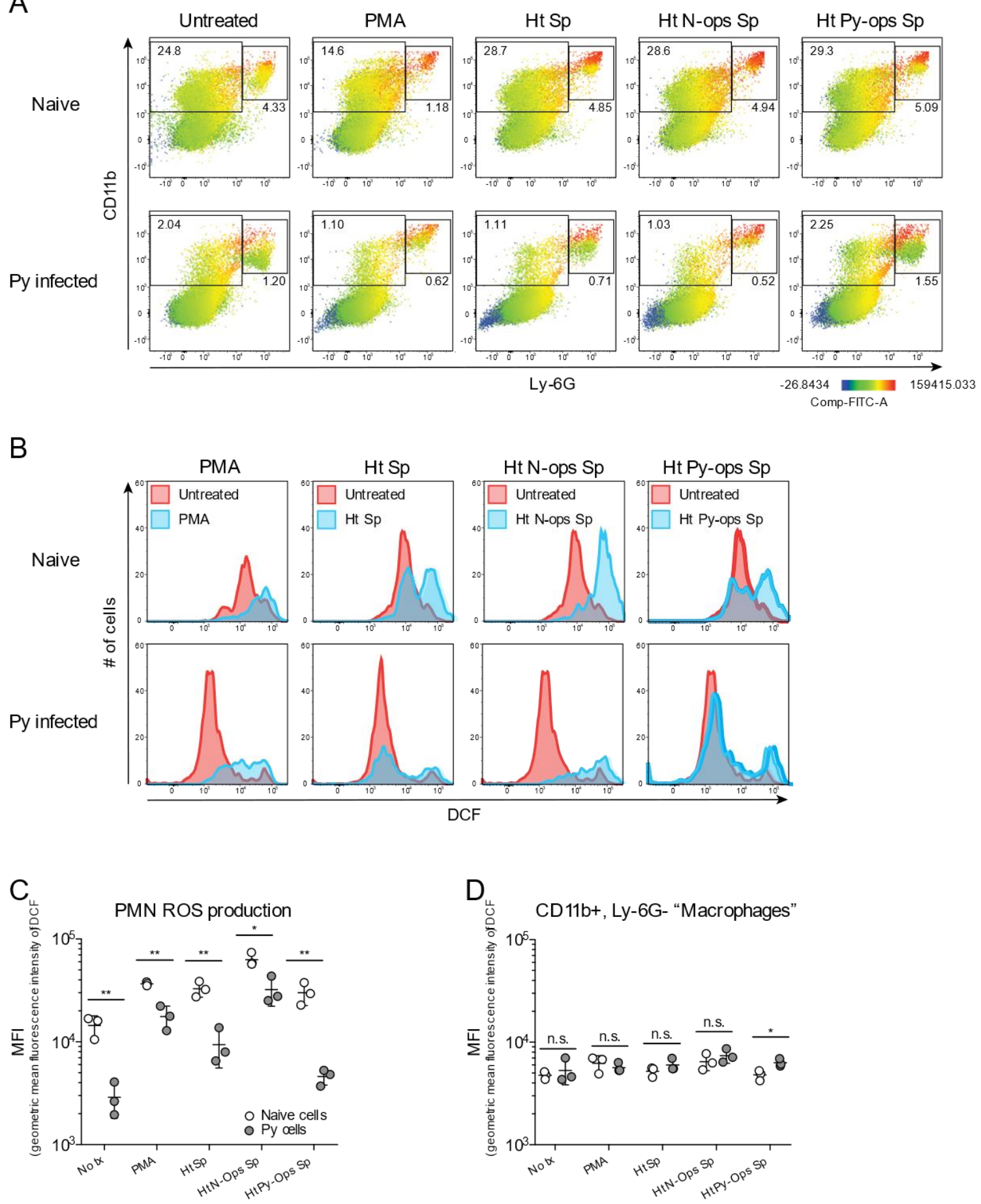

Figure 15. Splenic PMNs from $P$. yoelii infected mice have defective ROS production in response to heat-killed Sp. C57BL/6N mice were infected with $10^{5}$ Py pRBCs. Single cell suspensions from whole spleens of naïve and Py-infected mice (days 14 or 15 p.i.) were incubated with DFC to detect intracellular ROS. Cells were then treated with PMA, heat treated Sp (Ht Sp), or Ht Sp opsonized with 10\% serum from naïve or Py-infected mice. A) Heat map displays DCF fluorescence in CD11b x Ly6G cells to indicate amount of intracellular ROS. Heat maps are from an individual mouse in each group that are representative of a single experiment and of three total independent experiments. B) Histogram showing DCF fluorescence of splenic PMNs $\left(\mathrm{CD} 11 \mathrm{~b}^{+} / \mathrm{Ly} 6 \mathrm{G}^{+}\right)$as shown in box in (A). DCF geometric mean fluorescence 
intensity in PMNs $\left(\mathrm{CD} 11 \mathrm{~b}^{+} / \mathrm{Ly}_{6} \mathrm{G}^{+}\right)(\mathrm{C})$ and macrophages/monocytes $\left(\mathrm{CD} 11 \mathrm{~b}^{+} / \mathrm{Ly}^{6} \mathrm{G}^{-}\right)$(D) populations as indicated in (A). Each symbol represents an individual mouse. Data (mean \pm S.D.) are representative of three total independent experiments with 3 mice per group. Statistical significance was determined with multiple unpaired $t$-tests (D).

\section{Plasmodium yoelii infection impairs intracellular bacterial killing capacity of splenic}

\section{phagocytes}

To test impaired antibacterial responses at the cellular level, enrichment of splenic phagocytes was necessary because of the low overall abundance of macrophage, monocyte, and neutrophil subsets in the spleen, especially from Py infected mice ( less than $10 \%$ of total splenocytes during Py infection). Single cell suspensions of splenocytes from naïve control and Py-infected mice were enriched via depletion of $\mathrm{CD}^{+}$and $\mathrm{CD} 19^{+}$cells using a magnetic column. Figure 16A shows expression of $\mathrm{CD} 11 \mathrm{~b}$ and CD11c on all viable cells after column depletion. In order to further enrich for phagocytes, $\mathrm{CD} 11 \mathrm{~b}^{+} \mathrm{CD} 11 \mathrm{c}^{-}$cells were then sort purified as indicated by the gate in Figure 16B. Analysis of $\mathrm{CD} 11 \mathrm{~b}^{+} \mathrm{CD} 11 \mathrm{c}^{-}$splenocytes identified that about half were Ly$6 \mathrm{G}^{+}$cells (Naïve $=43.9 \% \pm 3.95$; Py-infected day $10=52.28 \% \pm 7.40$ ), indicating mixed populations of PMNs and mononuclear cells. Sort purified $\mathrm{CD} 11 \mathrm{~b}^{+} \mathrm{CD} 11 \mathrm{c}^{-}$cells were infected with Lm in a 5-hour gentamycin protection assay. Consistent with elevated bacterial burden in Py-infected mice, $\mathrm{CD} 11 \mathrm{~b}^{+} \mathrm{CD} 11 \mathrm{c}^{-}$cells from Py-infected mice exhibited impaired ability to kill intracellular Lm compared to cells from naïve mice (Fig. $16 \mathrm{C})$. 
A

Viable cells
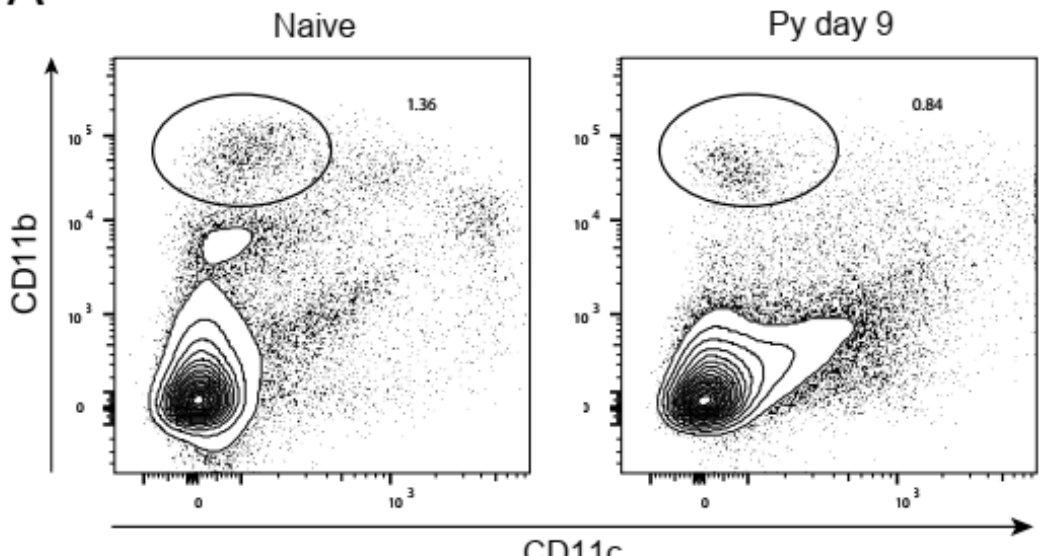

CD11c

B

Post-T cell and -B cell depletion
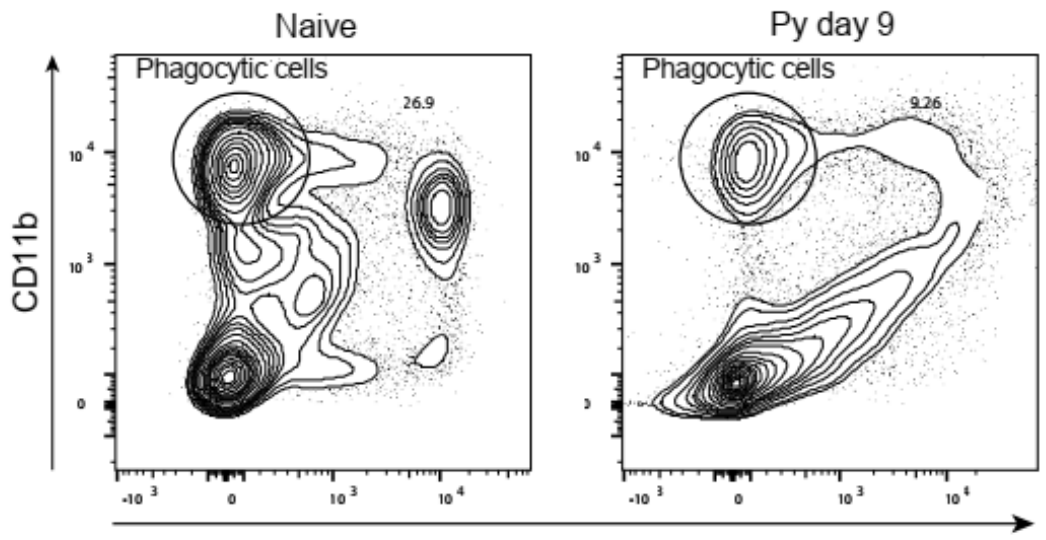

CD11c

C

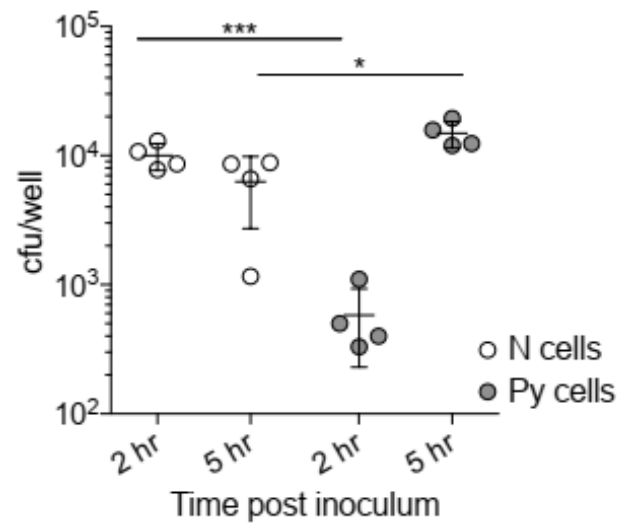

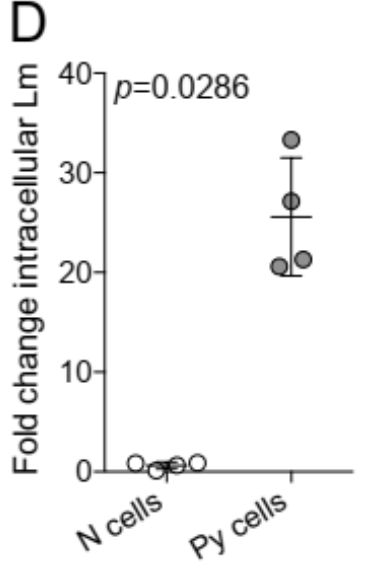

Figure 16. $P$. yoelii infection impairs antibacterial functions of splenic phagocytes. AC) After C57BL/6N mice were infected with $10^{5}$ Py pRBCs, splenic phagocytes were enriched from naïve or Py-infected mice day 9 post Py. Magnetic columns were used to enrich for splenic phagocytes by negative selection of $\mathrm{CD}^{+}{ }^{+} \mathrm{CD} 19^{+}$ cells. A) Contour plot showing CD11b and CD11c expression on viable splenocytes prior to $\mathrm{T}$ and $\mathrm{B}$ cell depletion on magnetic column. B) Contour plot showing CD11b and CD11c expression on splenocytes following magnetic column depletion of $\mathrm{T}$ and $\mathrm{B}$ cells. Circle indicates the population of splenic phagocytes that was sort purified for gentamicin protection assays. CD11 $b^{\text {hi }}$ CD11 $c^{-}$cells were then sort purified for experiments. $\mathrm{C}$ and D) $10^{5}$ sort-purified CD11 b ${ }^{\text {hi }} \mathrm{CD} 11 \mathrm{c}^{-}$splenic phagocytes were plated in each well and infected with Lm opsonized with homologous mouse serum with $\mathrm{MOI}=100$. Intracellular Lm burden was determined after 2 hours and 5 hours (C) in a gentamicin protection assay to evaluate fold change at 5 hours post infection (D). Fold change in intracellular

bacteria was determined by cfu/well at 5 hours post infection divided by cfu/well at 2 hours post infection. 


\section{$\underline{\text { Discussion }}$}

Due to the consistent outcome of elevated bacterial burdens in spleens of coinfected mice for both Lm and Sp models described in Chapter II, and the overall importance of this organ and the specific subsets of tissue-resident macrophages within during invasive bacterial infections, we sought to explore how populations of innate immune cells fluctuate over the course of $P$. yoelii infection. While other published work has shown decreases or even complete loss of some myeloid cell subsets in $P$. chabaudi or $P$. berghei murine malaria models, this Chapter aimed to characterize these changes in $P$. yoelii infection and describe difference between Py infected and naïve mice from day 10 post infection to 2 months beyond clearance of the infection. A major difference between the methods used in this work and previous work is that we used a flow cytometric analysis that allowed for quantitative measurement of surface marker expression on a single cell level, whereas other work was conducted on the somewhat qualitative method confocal microscopy. An advantage of using our flow cytometric method is that it allows us to examine how the total numbers of each population changed over time and how the relative abundance of each cell subset changed with respect to the total numbers of cells in each spleen. Also, compared to analysis of tissue sections via confocal microscopy, our method of cellular analysis by flow cytometry allows for quantitative measurement of a higher amount of cell surface markers, providing a more complete picture of splenic myeloid cell populations.

Due to the exhaustive nature of our splenic myeloid cell population analysis, we found no existing study that examined a similar multitude of cell types in the context of Plasmodium infection or any other disease. Therefore, many considerations were made in generating the panel of cell surface markers that were used to identify specific cell 
populations, choosing proper combinations of conjugated fluorochromes, and conceiving an informed gating strategy. In this manner, we took advantage of literature describing surface markers that are known to have specific levels of expression on cell population subsets of interest in order to improve the accuracy and power of our analysis. Characterizations of multiple cell populations within an organ or tissue are able to identify a specific cell type based on high expression or absence of a single surface marker that has been associated with an individual cell-type. In this work, however, several cell subtypes examined share nearly identical expression of multiple identifying surface markers due to their common hematopoietic origin. Since RPMs are known to be frustratingly autofluorescent $[157,158]$, they were the first cell type defined in our gating strategy immediately following the elimination of lymphocytes from the analysis, where CD3/CD19 positive cells separated (Fig. 11). To improve this cell analysis scheme in the future, the intrinsic autofluorescence of RPMs could be utilized by including and recording an open fluorescence channel, such as FITC, APC, or PE-Cy7, each of which have previously been demonstrated to have clear peaks that identify unstained RPMs as distinct from unstained dendritic cells as shown in Franken et. al [157] in Supplementary Figure 1. This method for identifying RPMs has also been demonstrated by Mitchell et. al 2012 [159].

Our results indicate that significant changes in several of the cell types examined occur during Py parasitemia and remain for up two months after parasite clearance. Among the cell types that are known to be important in clearance of invasive bacteria in the bloodstream, only MZMs were decreased in spleens of Py infected mice 101-102 days post Py infection. Lower numbers of MZMs at this time point correlates with 
impaired antibacterial innate immunity. Yet, MZMs were elevated in Py infected mice compared to control mice day 10 post Py infection when mice also displayed impaired bacterial clearance. Consequently, the initial increase and subsequent loss of MZMs in the spleen does not appear to change the bacterial clearance abilities in this model system.

There is strong rationale to hypothesize that one or more of the phagocytic cell populations characterized in this work become unable to perform anti-microbial functions during and following Plasmodium infection. While we were able to show defective control of intracellular replication in a more generally defined population of splenocytes from Py-infected mice that contained a mixture of macrophages, monocytes, and neutrophils (Figure 16), more work is needed to determine whether this defect is specific to a single cell type or if it is common to multiple. Similar results were obtained when using sorted splenic phagocytes from Py-infected mice on both days 9-11 (Figure 16) and day $\sim 30$ (data not shown) post infection in the ex vivo gentamicin killing assay, suggesting that there could be a common mechanism during acute parasitemia and at a time point shortly after parasite clearance. Chapter IV will seek to test the hypothesis that exposure to hemozoin is sufficient to impair control of intracellular Lm replication.

Another limitation of the in vitro gentamicin protection assay is the inability to determine whether a particular cell type has defective functions or if there is a general defect in multiple splenic phagocyte populations. Given that both Plasmodium chabaudi and Leishmania donovani infections induce loss of MZMs and MMMs, and Sp sepsis increases involvement of RPMs in their absence following L. donovani infection, there is rationale to specifically test the ability of RPMs to eliminate $\mathrm{Sp}$, and compare this to 
purified cultures of MZMs, MMMs, and mixed cultures [133, 134]. This type of experiment would potentially show stronger phenotypes if there are cells with normal functional ability diluting and diminishing the effect. Knowledge of functional capacity of specific cell types to control bacteria in vitro would provide meaningful insight into the potential mechanisms of the enhanced mortality we've observed in our Py+Sp coinfection model. There are, however, other factors that may contribute to the impaired clearance of Lm at early and late time points post infection, respectively. For example, loss of functional complement components during the parasite infection could decrease bactericidal capacity of the innate immune cells despite the initial increase in MZMs that would otherwise aid in bacterial clearance under normal conditions. Additionally, at time points that follow clearance of Py, complement factor function returns to normal (only observed in P. berghei/chabaudi), yet at this time the loss of total numbers of MZMs may be responsible for the long-term impaired clearance of $\mathrm{Lm}$. Further experiments are needed in order to completely understand exactly which anti-bacterial mechanisms of which specific cell types are impaired at different timepoints in this model system.

Additionally, our analysis is not able to detect changes in the ability of migratory innate immune cell populations to infiltrate the spleen in response to a secondary bacterial infection. There is strong evidence suggesting that monocyte, neutrophil, and dendritic cells have impaired chemotaxis $[117,160]$, antigen presentation, and other antimicrobial functions in the context of Plasmodium infections [61, 161-164]. It is likely that the during/after Plasmodium infection the inability of these cells to migrate/egress from circulation into the spleen impedes the clearance and overall immunity to bacterial coinfections 
Prior studies characterizing changes in populations of splenic myeloid cells during disease have utilized confocal microscopy of spleen sections, which allows for characterization of splenic architecture and the position of cells relative to landmarks such as red pulp, white pulp, marginal zones, and germinal centers [134]. While this approach is useful for evaluating the stage of maturation or extent of adaptive immune responses, it is only partly quantitative and therefore does not provide a full picture of spleen functions. Splenomegaly and disruption of marginal zone architecture during malaria makes analysis of cell types based on location in tissue problematic due to a lack of important landmarks. We chose to use a flow cytometry method for analysis of splenic innate immune cell populations because it allowed us to take advantage of differential expression of surface markers to identify specific subsets, such as MMMs and MZMs, without limiting the number of cell types we could explore in a single sample.

The gating strategy and definitions of cell populations was developed by combining strategies from previous studies - for neutrophils, eosinophils, dendritic cells, inflammatory monocytes, and red pulp macrophages [165]. Since no other study had previously characterized MMMs and MZMs with flow cytometry, a method for detecting these cells was devised by drawing gates based on the fluorescence-minus-one(FMO) samples, which were stained with all antibodies other than the one being examined. Discrepancies in background autofluorescence of PE and APC (recognizing CD169 and CD209b and identifying MMMs and MZMs, respectively) were evident between samples from naïve and Py-infected mice at all timepoints post Py infection and gates were drawn accordingly for all samples in respective naïve or Py infected mouse groups independently for each experiment/repeat. In each experiment cells from Py-infected 
mice appeared to have higher background autofluorescence in APC and PE channels than cells from naïve mice despite the trend of these markers being expressed more highly in cells from naïve mice (data not shown). Whether or not this observation was occurring in other cell types/fluorophore channels was not assessed, and further analysis should be done to determine the overall impact it may have had on these results. One explanation for this observation that convoluted the analysis is that some factor related to the Py infection increased the overall autofluorescence of MMMs, MZMs, and possibly other cell populations, which would potentially impact absolute and relative numbers of every cell type that was examined.

The activity of Plasmodium-induction of serine proteases presents another potential explanation of both the observed loss of splenocyte subpopulations during some but not all timepoints analyzed post-Py infection, and the technical problem of inconsistent baseline (unstained) levels of MMM and MZM markers. During the blood stage of Plasmodium infection both parasite and host immune cells produce factors, such as Plasmodium serine proteases and neutrophil elastase (also a serine protease), that are capable of cleaving surface molecules from innate immune cells, including toll-like receptors, and intracellular adhesion molecule-1(ICAM-1/MAC1/CD54) [166, 167]. This receptor-cleaving activity may be occurring during Py infection and cleaving immune cell surface proteins that are not yet known to be affected. In this scenario, the impact of proteases on immune cell surface markers may vary over the course of infection with cleavage decreasing as the course of the infection nears senescence and eventually having no affect. Hypothetically, this situation would have an immense impact 
on our analysis and accounting for this phenomenon would be essential for accurate comparisons between naïve and Py-infected mouse splenocyte populations.

Previous work from both clinical studies and animal models describe impaired ROS production by PMNs isolated from Plasmodium-infected individuals and mice [61, 62, 64]. These reports linked the lack of sufficient respiratory burst observed during acute severe malaria cases as well as months after senescence to the clinical observations that demonstrated an elevated susceptibility to invasive NTS coinfections $[53,56,168$, 169]. The experiments performed in these investigations measured ROS production from neutrophils isolated peripheral blood or bone marrow following stimulation with PMA, in one case, $1 \times 10^{8}$ cfu of opsonized $S$. typhimurium in vitro [64]. To date, defective neutrophil functions, including impaired ROS production, chemotaxis, and general impairment of bacterial killing - is attributed to the activity of HO-1 related to Plasmodium infection induction of hemolysis $[61,62,64]$. While the impaired ability of PMNs to produce sufficient level of ROS needed to kill intracellular NTS was clearly demonstrated, these studies raise several questions. Both Cunnington et al. [61] and Nyirenda et al. [64] detected decreased ROS production in PMNs from patient samples obtained either 4 to 8 weeks after the initial check and treatment with artemetherlumefantrine or other anti-malarial drugs.

In this work we sought to examine ROS production in myeloid cells from spleens from Py infected mice compared to naïve mice. Since parasite-dependent hemolysis occurs only during active replication of the parasite in the erythrocytic stage of infection, and we had found impaired clearance of Lm and increased susceptibility to Sp during Py coinfection (Figures 4 and 8 in Chapter II), we hypothesized that factors other than HO-1 
and hemolysis may be contributing to the Plasmodium-dependent defect in PMN ROS production. Additionally, we sought to characterize ROS production in myeloid cells from the spleen, an organ of great importance during invasive bacterial infections [39, $124,156]$, rather than from peripheral blood. Also, we hoped to determine whether the impaired ROS production occurred in macrophages and monocytes within the spleen, or if this defect was specific to PMNs. Finally, our ex vivo assay was designed to test intracellular ROS production of splenocytes in response to Lm and Sp, with the addition of PMA stimulation to serve as a positive control.

We found that Py infection significantly impaired ROS production by PMNs, but not "macrophages" (CD11b-, Ly-6G-), when treated with PMA, Lm, and heat-treated Sp. It is known that PMN production of ROS is sufficient to kill intracellular Lm [154], and therefore, this result supports our hypothesis that Py-infection induced reduction in PMN ability to produce ROS contributes to the impaired clearance of Lm in spleens of coinfected mice. We also observed reduced ROS production of splenic PMNs from Pyinfected mice in response to heat-treated $\mathrm{Sp}$. This defect likely does not affect killing or clearance of $\mathrm{Sp}$ since it is know that Pneumococcus can survive significant levels of oxygen radicals and in fact produces hydrogen peroxide $\left(\mathrm{H}_{2} \mathrm{O}_{2}\right)$, which acts together with pneumolysin toxin to kill host cells independent of caspases [170], and disrupts alveolar epithelium as a mechanism to initiate firm adherence while invading the respiratory tract [171]. It is possible, however, that the decreased ROS production in splenic PMNs from Py-infected mice is a symptom of a larger defect in anti-microbial functions. This idea will be explored further in Chapters IV and V. 
Py infection may also impair the innate immune response to invasive bacteria via serum-dependent effects. Complement components $\mathrm{C} 1 \mathrm{q}$ and $\mathrm{C} 3$ interact with parasitized RBCs during P. falciparum infection, initiating the complement cascade, which leads to reduced serum levels of $\mathrm{C} 1 \mathrm{q}$ and lower complement activity compared to uninfected individuals $[120,155]$. Several components of the complement system are considered essential for immunity to Sp infection, as individuals with genetic defects in C3, which is involved in both the classical and alternative complement pathways, experience recurring pneumococcal infections and infections with other encapsulated bacteria. Similarly, mice lacking expression of $C 3$, factor $\mathrm{B}, \mathrm{Cl} q a$, or $C 4$ genes are more susceptible to $\mathrm{Sp}$ infections [153]. Moreover, killing of Sp by neutrophils has been shown to require active complement opsonophagocytosis via CR3 (CD11b/CD18 heterodimer complement receptor) [172]. Consistent with Plasmodium-induced depletion of complement and a role for complement in immunity to Sp, we observed decreased ROS production in splenic PMNs from naïve mice stimulated with heat-killed Sp opsonized with serum from Pyinfected mice compared to splenic PMNs stimulated with heat-killed Sp opsonized with serum from naïve mice (Fig. 15A-C). Similarly, PMNs from Py-infected mice showed increased ROS production following stimulation with heat-killed Sp opsonized with serum from naïve mice compared to serum from Py-infected mice. This observation could indicate a serum factor-dependent defect in neutrophil opsonophagocytosis that may lead to impaired immunity to Sp during malaria. Although ROS bursts within neutrophil phagolysosomes are not sufficient to kill Sp [172], deficiency in PMN ROS production in response to heat-killed Sp opsonized with serum from Py-infected mice compared to heat-killed Sp opsonized with serum from naïve mice suggests that 
phagolysosome maturation, or other events that follow opsonophagocytosis, may be inhibited or less responsive. It has also been shown that serum from febrile P. falciparum infected individuals results in decreased C3 deposition on NTS compared to serum from non-febrile $P$. falciparum negative individuals [63]. Collectively, these observations suggest that complement depletion during Plasmodium infections may be a general deficiency associated with invasive bacterial infections.

To better understand the interaction between phagocytic cells of the spleen and $\mathrm{Lm}$ in the context of malaria, the gentamicin protection assay was developed. This technique allowed us to compare intracellular bacterial burdens in cells from spleens of Py-infected and naïve at various times post-infection to determine whether Plasmodium infection effected the cell's ability to control intracellular replication (Fig. 16). At the early timepoint of 2 hours post Lm infection, fewer CFUs were detected in cells from Py infected mice. This result can be interpreted in several ways. First, a difference in Lm adherence could alter either uptake of Lm by phagocytosis or Lm invasion of cells in this assay. Since gentamicin was added to the culture 1 hour after infection, attached Lm and other extracellular Lm are killed and differences in Lm attachment to the surface of cells from naïve and Py-infected at this time would no longer impact the resulting bacterial burdens 1 or 4 hours later when bacteria was plated for cfu analysis. It may be that splenic phagocytes from Py-infected mice have some modified surface components that impede Lm attachment, and subsequent invasion or uptake. Alternatively, changes in serum factors during Py infection, such as opsonins: antibody and complement factors, could change dynamics of Lm ability to induce its uptake. Some other factors that could influence numbers of intracellular bacterial burdens after 2 hours are differences in 
extracellular and intracellular killing. Neutrophils encountering Lm in these in vitro conditions would be capable of producing bactericidal granules and NETs. They would also have adequate time to internalize $\mathrm{Lm}$ and fuse $\mathrm{Lm}$-containing phagosomes with granules and combining anti-microbial components such as proteases and ROS. Therefore, lower CFUs in cells from Py-infected mice may have resulted in increased ability of these cells to kill extracellular Lm, intracellular Lm, or both. Finally, the decreased bacterial burdens seen in cells from Py-infected mice could be explained by a general defect in Lm phagocytosis, which has been previously described in the context of malaria and hemozoin $[85,173,174]$.

While results at 2 hours post $\mathrm{Lm}$ infection indicated lower intracellular $\mathrm{Lm}$ burdens in cells from Py-infected mice, CFU analysis at 5 hours post-Lm infection showed a drastic increase compared to almost no change in cells from naïve mice (Fig. 16 C). This result indicates that cells from Py-infected mice, despite having lower Lm burdens at 2 hours post infection, were permissive to Lm growth between 2 and 5 hours, whereas cells from naïve mice were capable of controlling Lm intracellular replication (Fig. 16 C and D). This result introduces several important questions pertaining to the Py-dependent mechanism(s) that are rendering splenic phagocytes into permissive to bacterial growth. Previous reports that investigated Plasmodium coinfections with NTS have found that Py hemolysis-induced HO-1 and IL-10 inhibit essential functions of innate immune cells and are sufficient to increase the overall severity of NTS infection $[59,61,62,64]$. In the case of immunity to $\mathrm{Lm}$ in our Py+Lm coinfection model, however, it is unlikely HO-1 or IL-10 are contributing to the defective control of Lm growth as we found that inhibiting either of these factors did not improve 
Lm clearance in coinfected mice (Fig. 6). Lm is susceptible to intracellular production of ROS within phagocytic cells, specifically neutrophils [175]. We found that PMNs from Py-infected mice have defective ROS production in response to Lm (Fig. 14). Additional experiments are required to determine whether defective ROS production is contributing to this phenotype and if the defect is specific to a particular cell type (monocyte, macrophage, and/or neutrophils), as the cells sorted for this assay contained a mixture of all CD3/CD19 ${ }^{--}, \mathrm{CD} 11 \mathrm{c}^{-}$and $\mathrm{CD} 11 \mathrm{~b}^{+}$, which should only eliminate all lymphocytes, and some types of dendritic cells. The impact of $\mathrm{Hz}$ on this system is explored in Chapter IV. 


\section{CHAPTER IV}

\section{THE PARASITE FACTOR HEMOZOIN IMPAIRS INNATE IMMUNITY TO BACTERIAL COINFECTIONS DURING MALARIA}

\section{Introduction:}

During the blood stage of Plasmodium infection, parasites within infected erythrocytes release proteases to digest protein components of hemoglobin within their digestive vacuoles resulting in the release of free heme, which is toxic to the parasite. Heme is detoxified within the parasite digestive vacuole via non-enzymatic polymerization into hemozoin $(\mathrm{Hz})$, which is an insoluble crystal structure[176]. Following erythrocyte rupture and merozoite escape, $\mathrm{Hz}$ is released into circulation and engulfed by phagocytic cells resulting in deposition in tissues and organs such as spleen, liver, brain, lungs, and bone marrow. Hz deposition following Plasmodium berghei infection was evident in the spleen, liver, bone marrow, and peripheral blood 196 days after infection [110]. Hz has also been reported in lung and brain tissue of P. berghei and Plasmodium chabaudi infected mice [83] and is involved in the malaria-driven pathologies specific to those organs, such as malaria-associated acute respiratory distress syndrome and cerebral malaria, respectively $[81,177,178]$.

The immune modulatory potential of $\mathrm{Hz}$ has been examined in several studies with a wide variety of $\mathrm{Hz}$ preparations and cell types being used. While synthetically generated $\mathrm{Hz}$ has been described in some cases as "immunologically inert", others have 
found $\mathrm{Hz}$ formed naturally during erythrocyte infection to alter and impair many immune cell functions [179]. For example, uptake of parasitized RBCs or purified $\mathrm{Hz}$ by human monocyte-derived macrophages causes impaired phagocytosis of additional RBCs or production of a PMA-induced oxidative burst [173], which are impaired via Hzdependent inactivation of protein kinase C and NADPH-oxidase [164, 180]. Purified digestive vacuoles with intact membranes (not sonicated or detergent treated) isolated from $P$. falciparum-infected RBC culture have been shown to activate complement, and decrease ROS production and bactericidal capacity of human PMNs [161, 162]. Internalization of $\mathrm{Hz}$ has also been found to induce the production of pro-inflammatory cytokines such as IL-1 $\beta$ and TNF and also increase chemokine production and enhance expression of their receptors (CCL3-5, MCP-1) in mouse macrophages and human peripheral blood monocytes [181, 182]. Antigen presentation functions and overall functional maturation of dendritic cells are also impacted, as decreased surface expression of CD54, CD11c, and MHC-class II in human PBMCs following stimulation with IFN- $\gamma$ has also been observed during co-incubation with $\mathrm{Hz}$ [163].

Few studies, however, have looked directly at the interactions between innate immune cells and $\mathrm{Hz}$ in the context of in vivo bacterial infection or the effect of $\mathrm{Hz}$ on neutrophil function, and we have found none to date that have explored interactions with Streptococcus pneumoniae infection. Moreover, previous work has shown impaired antimicrobial functions of innate immune cells from African children with uncomplicated malaria that lasted up to 8 weeks after antimalarial treatment [61]. Of note, there is unlikely to be parasite-induced hemolysis during convalescence, which is the current hypothesis by which Plasmodium suppresses anti-bacterial innate immunity. Collectively, 
this leads to the hypothesis that Hz contributes to Plasmodium-induced suppression of innate immunity to bacterial infections.

The aim of this Chapter is to explore the immunomodulatory properties of $\mathrm{Hz}$ in the context of bacterial infections and the innate immune cell functions that are vital to controlling invasive bacterial infection. To test these hypotheses, a novel method of isolating and purifying hemozoin from livers and spleens of Py-infected mice (PyHz) was developed. The addition of $\mathrm{PyHz}$ to cultures of innate immune cells in vitro and injecting intravenously into naïve mice prior to bacterial infection allowed us to gain insight into the interaction between this parasite metabolic byproduct and how bioactive molecules associated with $\mathrm{Hz}$ contribute to Plasmodium-induced immunosuppression.

\section{$\underline{\text { Results }}$}

Impact of Plasmodium-derived hemozoin on anti-bacterial functions and control of intracellular bacterial replication in splenic phagocytes

Hemozoin has been shown to remain in host tissues, including the spleen, for many months after the clearance of infection $[83,110]$. This physical characteristic of $\mathrm{Hz}$ and the long-term suppression of innate immunity to bacteremia following clearance of Plasmodium yoelii (Fig. 4C), led to the hypothesis that $\mathrm{Hz}$ is a parasite-derived factor that is capable of impairing anti-bacterial effector functions. To perform these studies, we developed an approach to isolate $\mathrm{Hz}$ from the spleens and livers of mice that had cleared Py infections (Figure 17). An image of $\mathrm{Hz}$ formed in food vacuole of $P$. falciparum is shown in Figure 17A [183]. After collecting the organs (spleens of naïve and Py-infected mice shown in picture Figure 17B) they were thoroughly homogenized and then washed in de-ionized water and then passed through a 100-micron nylon mesh filter to remove 
remaining pieces of tissue. Next hemozoin was suspended in PBS before being added to the top of a discontinuous Percoll density gradient with $40 \%$ and $80 \%$ fractions (Fig. 17 C). Hemozoin was collected from the interface between the 40 and $80 \%$ fractions as well as beneath the $80 \%$ fraction. Isolated hemozoin from $\mathrm{Py}$-infected mouse organs ( $\mathrm{PyHz})$ was finally washed in $2 \mathrm{mM}$ mannitol-containing buffer and resuspended in sterile saline prior to use. Details of other Hz preparations can be found in the Materials and Methods section.

A.

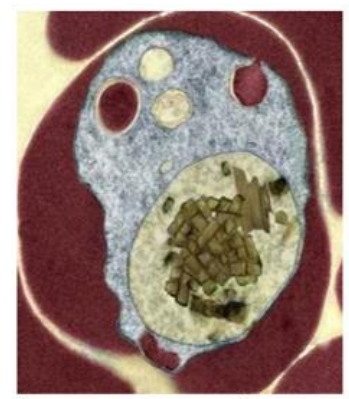

B.

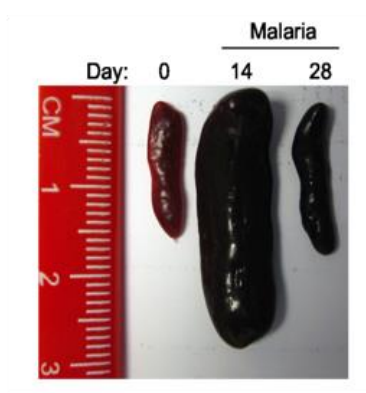

C.

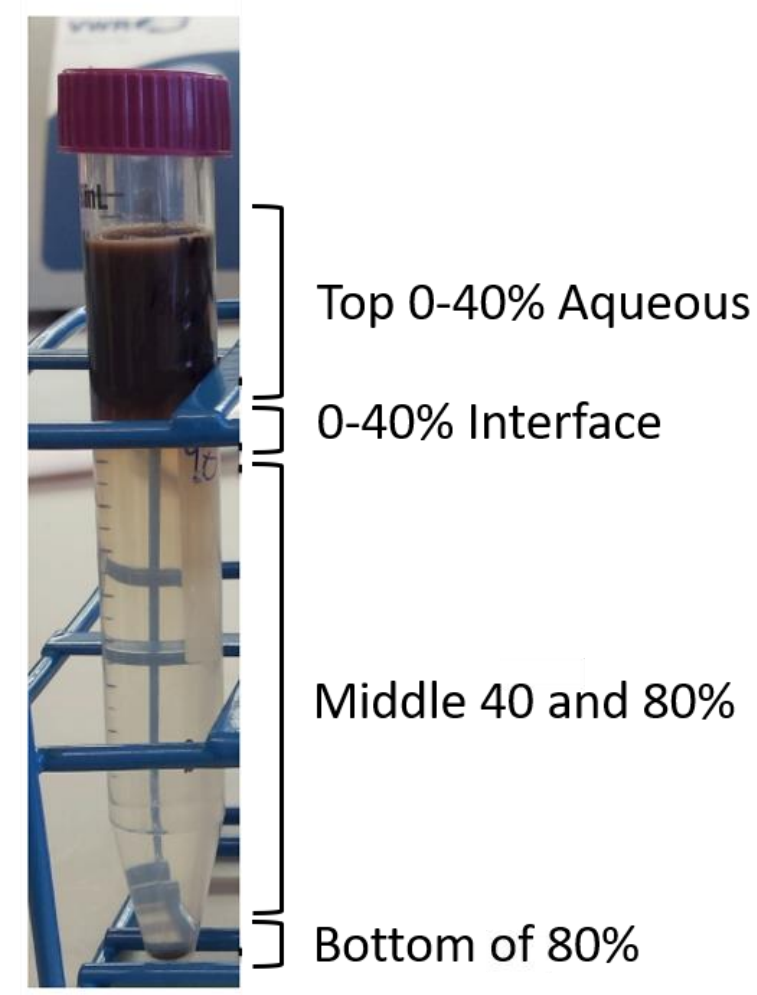

Figure 17. Hemozoin isolation from liver and spleens of Py-infected mice. A) Hemozoin crystals within the digestive vacuole of $P$. falciparum, color was added with imaging software (adapted from D. Jani et al. 2008 [183]. B) Spleens from naïve, 14, and 28 day Py-infected mice. Dark color in Py-infected spleens due to $\mathrm{Hz}$ deposition. Adapted from C) Percoll gradient used for isolation of hemozoin collected from homogenized spleens and livers from Py-infected mice. 
To test the hypothesis that $\mathrm{Hz}$ contributes to Py-induced immune suppression, we first examined whether $\mathrm{Hz}$ contributed to the defective production of ROS that was observed in splenic PMNs isolated from Py-infected mice (Fig. 14 and 15). Single cell suspensions from whole spleens isolated from naïve mice were incubated with $\mathrm{PyHz}$ for 6 or 16 hours prior to stimulation with Lm, Sp, or control PMA (Fig. 18). One hour after stimulation with bacteria or PMA, cells were treated with DCF and intracellular ROS was measured via flow cytometry. Low levels of ROS were detected in both untreated cells and cells treated with PyHz alone (Fig. 18A,C-D). While PMA stimulation induced similar ROS in untreated and PyHz treated PMNs, opsonized Lm and heat-treated opsonized Sp did not elicit a robust ROS response (Fig. 18A-D). In contrast, both untreated and PyHz-treated PMNs infected with live-opsonized Sp had high levels of ROS (Fig. 18A-D), with PyHz-treated PMNs exhibiting significantly higher ROS than untreated samples (Fig 18D). Similar results were observed in the spleen CD11b $/$ Ly6G' populations (Fig. 18E). 

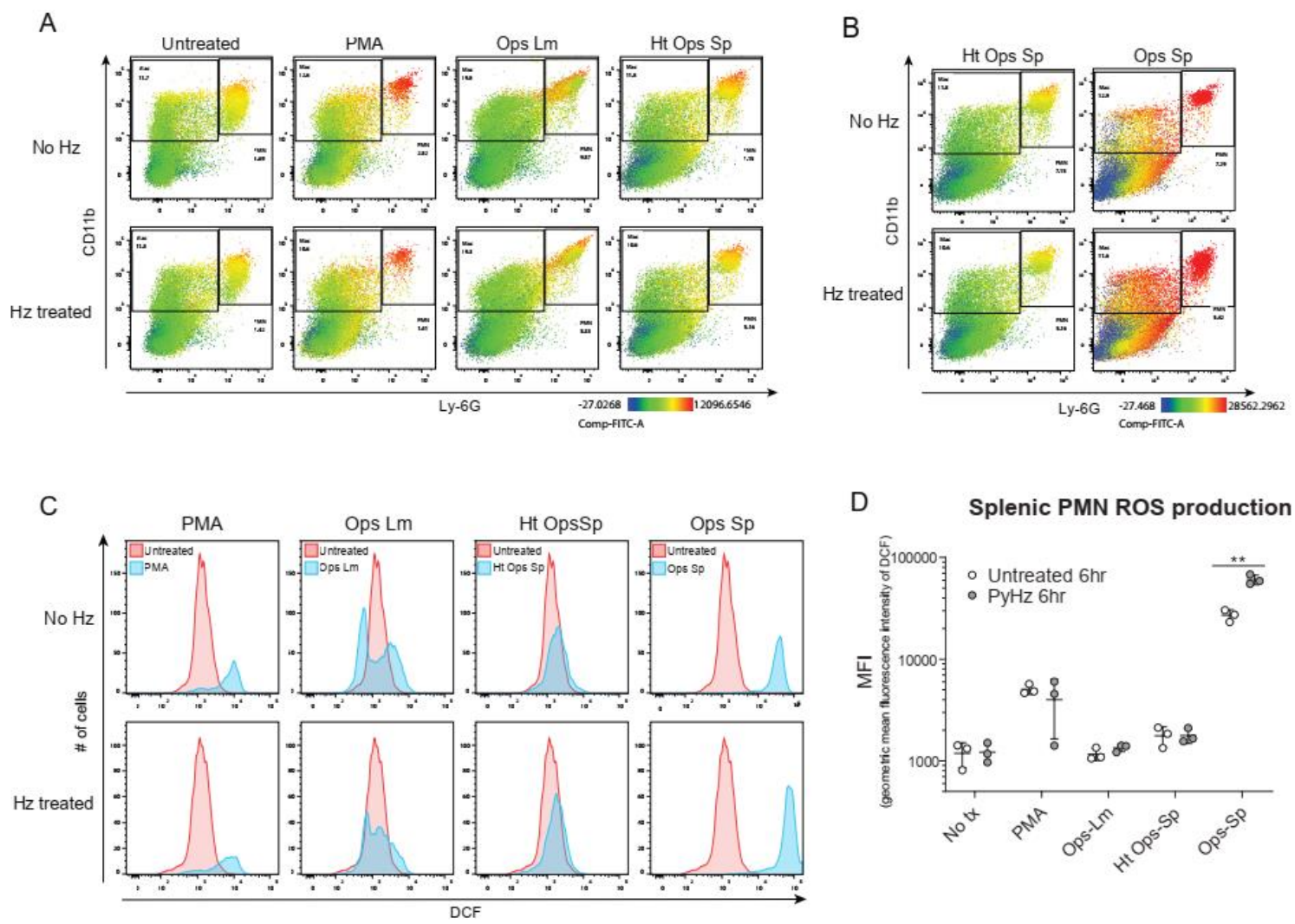

$E$

Figure 18. PyHz treatment does not alter intracellular ROS production of splenic neutrophils in whole spleen single cell suspension. Single cell suspensions from whole spleens of naïve mice were incubated with $60 \mu \mathrm{g}$ hemozoin isolated from liver and spleen homogenate from Py-infected mice $(\mathrm{PyHz})$ for 6 or 16 hours. After incubation with $\mathrm{PyHz}$, cells were incubated with DFC to detect intracellular ROS. Cells were then treated with PMA, opsonized $\triangle a c t A \mathrm{Lm}$ (Ops-Lm), heat treated opsonized Sp (Ht Ops-Sp), or opsonized live Sp (Ops-Sp). A and B) Heat map displays DCF fluorescence in CD11b x Ly6G cells to indicate amount of intracellular ROS. A) and B) display the same data for the Ht Ops-Sp group, B) includes the live Ops-Sp group with an increased maximum DCF signal for improved visualization of changes in ROS production. Heat maps are from an individual mouse in each group that are representative of a single experiment and of three total independent experiments. C) Histogram showing DCF fluorescence of splenic PMNs $\left(\mathrm{CD} 11 \mathrm{~b}^{+} / \mathrm{Ly}^{6} 6 \mathrm{G}^{+}\right)$as shown in box in (A). DCF geometric mean fluorescence intensity in PMNs $\left(\mathrm{CD} 11 \mathrm{~b}^{+} / \mathrm{Ly}^{6} \mathrm{G}^{+}\right)(\mathrm{D})$ and macrophages/monocytes $\left(\mathrm{CD} 11 \mathrm{~b}^{+} / \mathrm{Ly}_{6} \mathrm{G}^{-}\right)(\mathrm{E})$ populations as indicated in (A and B). Each symbol represents an individual mouse. F) DCF geometric mean fluorescence of untreated and PyHz-treated PMNs $\left(\mathrm{CD} 11 \mathrm{~b}^{+} / \mathrm{Ly} 6 \mathrm{G}^{+}\right)$as shown in box in (A) incubated in the indicated conditions for 6 and 16 hours prior to DCF treatment and PMA stimulations or infections. Data (mean \pm S.D.) are representative of three total independent experiments with 3 mice per group for (D - F). Statistical significance was determined with multiple unpaired $t$-tests (D). 
Since we did not observe differences in ROS production between PyHz treated and untreated PMNs, or other cell populations from whole spleen single cell suspensions, a purified population of neutrophils were isolated from bone marrow of naïve mice and incubated with $\mathrm{PyHz}$ prior to infection with $\mathrm{Lm}$ and $\mathrm{Sp}$ in order to increase the amount of interaction between our cells of interest and the Hz. The purity of neutrophils in the population was determined based on the $\mathrm{Ly}-6 \mathrm{G}^{+} / \mathrm{CD} 11 \mathrm{~b}^{+}$gate drawn in Figure $19 \mathrm{~A}$ and compared to total single cells (Fig. 19C). These conditions were also performed with hemozoin isolated from red blood cell cultures of the important human pathogen, $P$. falciparum $(\mathrm{PfHz})$. Following incubation with $\mathrm{Hz}$, cells were treated with DCF and then stimulated with PMA or with opsonized Lm, Ht Ops Sp, or live Ops-Sp. Similar to what was observed using whole spleen single cell suspensions, incubation with $\mathrm{PyHz}$ or $\mathrm{PfHz}$ did not impair intracellular ROS production in bone marrow purified neutrophils (BMNs) (Fig. 19). Increased ROS in cells stimulated with live opsonized Sp was seen again in this experiment, comparable to the result in Figure 18 with whole spleen single cell suspension and $\mathrm{Hz}$ treatment. Purified BMNs with $\mathrm{PyHz}$ or PfHz treatment contained high levels of ROS when stimulated with live opsonized Sp (though not as high as in PMNs in whole spleen single cell suspension), yet BMNs that were not treated with $\mathrm{PyHz}$ or PfHz displayed similar DCF signal as unstimulated cells following infection with live opsonized Sp (Fig. 19D). Together, these data indicate that the ability of BMNs as well as PMNs from whole spleen single cell suspensions to produce ROS is not impeded by $\mathrm{Hz}$. 
A

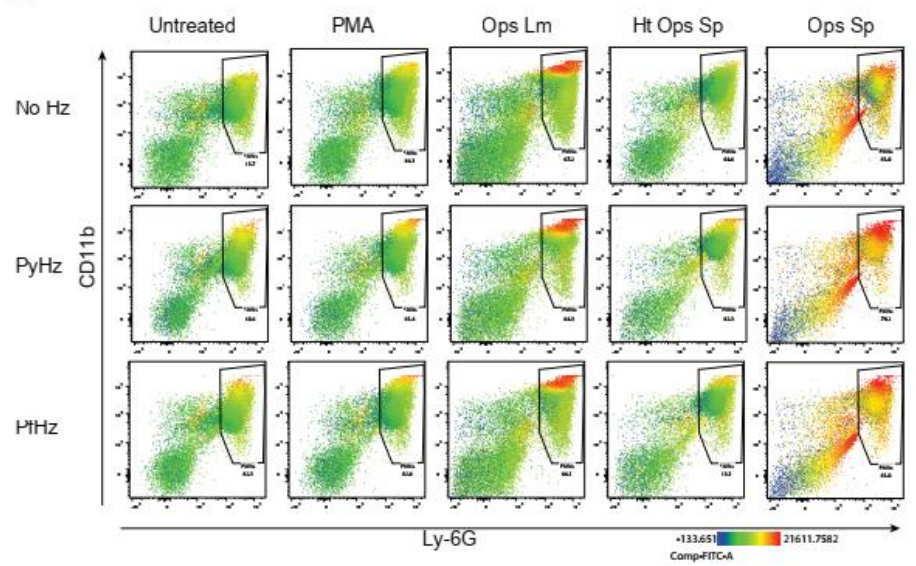

B

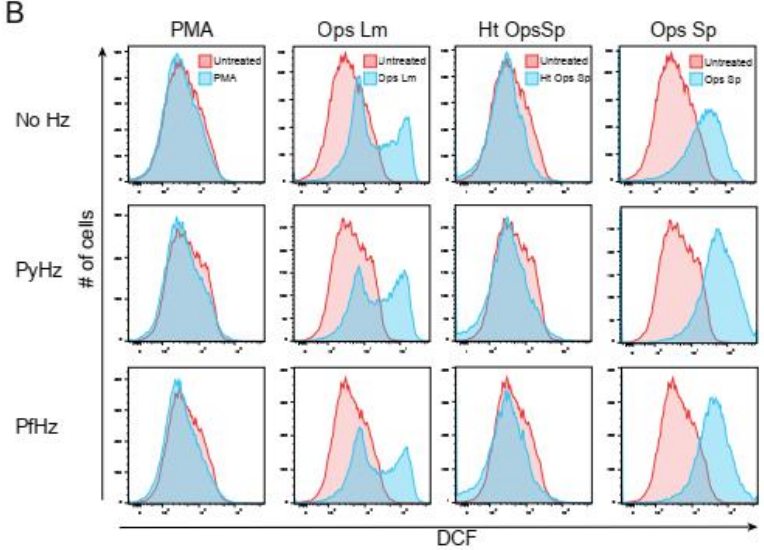

C

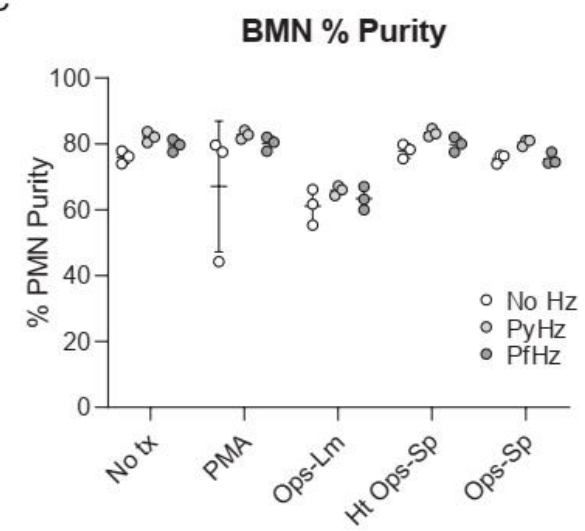

D

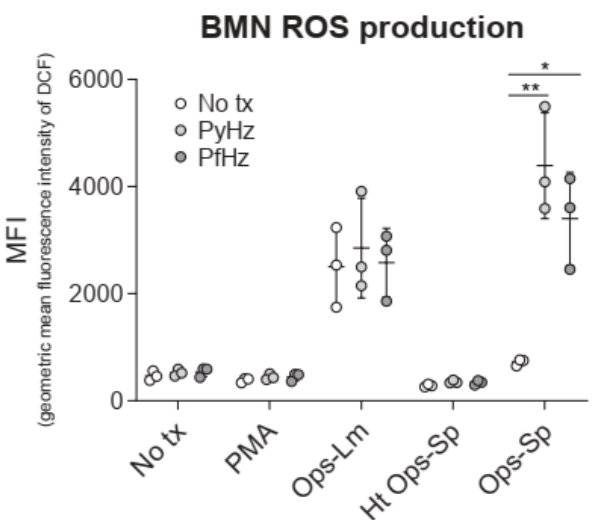

Figure 19. Hemozoin treated bone-marrow-purified Neutrophils do not have defective ROS responses. Purified bone marrow neutrophils (BMNs) were isolated from naïve mice and incubated with $60 \mu \mathrm{g}$ of either hemozoin isolated from liver and spleen homogenate from Py-infected mice $(\mathrm{PyHz})$ or hemozoin isolated from Plasmodium falciparum-infected human red blood cell culture $(\mathrm{PfHz})$. After incubation with $\mathrm{PyHz}$ or PfHz for 4-5 hours, cells were incubated with DFC to detect intracellular ROS. Cells were then treated with PMA, opsonized $\triangle a c t A$ Lm (Ops-Lm), heat treated opsonized $\mathrm{Sp}$ (Ht Ops-Sp), or opsonized live Sp (Ops-Sp). A) Heat map displays DCF fluorescence in

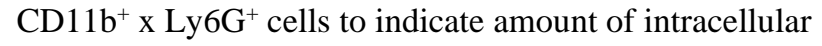

ROS. Each heat map layout is from an individual mouse in each group that are representing one of three independent experiments. B) Histogram showing DCF fluorescence of purified BMNs $\left(\mathrm{CD} 11 \mathrm{~b}^{+} / \mathrm{Ly} 6 \mathrm{G}^{+}\right)$as shown in the gate in (A). DCF geometric mean fluorescence intensity in BMNs $\left(\mathrm{CD} 11 \mathrm{~b}^{+} / \mathrm{Ly}^{6} \mathrm{G}^{+}\right)(\mathrm{C})$. Percent purity of BMNs in each sample determined as percent of $\mathrm{CD} 11 \mathrm{~b}^{+} / \mathrm{Ly} 6 \mathrm{G}^{+}$single cells (D). Each symbol represents an individual mouse. ed in box in (A) incubated in the indicated conditions for 6 and 16 hours prior to DCF treatment and PMA stimulations or infections. Data (mean \pm S.D.) are representative of three total independent experiments with 3 mice per group for (C and D). Statistical significance was determined with one-way ANOVA followed by Tukey's multiple comparisons test. 
In order to assess the ability of parasite derived hemozoin to contribute to impaired innate immunity to bacterial coinfections, we sought to create a coinfection model that utilizes a Plasmodium parasite that is deficient in $\mathrm{Hz}$ production. Mutant strains of $P$. berghei $(\mathrm{Pb})$ NK65 were obtained to determine whether the absence or a significant decrease of $\mathrm{Hz}$ production during Plasmodium infection would improve clearance of Lm compared to wild-type $\mathrm{Pb}$ NK65. We acquired $\Delta p m 4$, a Pb NK65 mutant strain that lacks plasmepsin 4 ( $\mathrm{pm} 4)$, a gene encoding a protease involved in digesting hemoglobin [184]. Given the importance of $\mathrm{Hz}$ formation in the detoxification of heme within infected $\mathrm{RBCs}, \mathrm{Pb}$ strains with impaired $\mathrm{Hz}$ production have an attenuated phenotype characterized by a significantly reduced parasitemia during infection [184]. To ensure that decreases in parasitemia were not responsible for any phenotypes observed, we also obtained a $\mathrm{Pb}$ strain lacking a gene encoding dipeptidyl aminopeptidase 2 ( $\triangle d p a p)$ that exhibits attenuated parasitemia similar to $\Delta p m 4$. These strains were kindly provided by Dr. Phillipe Van den Steen from KU Leuven-University of Leuven, in Leuven, Belgium. Mice were given intraperitoneal injections with $10^{4}$ pRBCs of the wild type Plasmodium berghei strain NK65 (Pb wt), Pb $\Delta$ dpap, and $\Delta p m 4$ obtained from infected donor mice and parasitemia was measured on days 6,8 , and 9 . On day 7 post $\mathrm{Pb}$ infection, injections of $5 \times 10^{6} \mathrm{CFU}$ of $\triangle a c t A \mathrm{Lm}$ were administered to $\mathrm{Pb}$-infected mice and naïve controls. As expected, mice infected with $\mathrm{Pb} \Delta d p a p$, and $\Delta p m 4$ strains had lower parasitemia than wild type (Fig. 20A). While $\Delta p m 4$ infected mice had significantly less $\mathrm{Hz}$ than WT in both liver and spleens by day 9 post $\mathrm{Pb}$ infection, $\mathrm{Hz}$ levels in $\triangle d p a p$ infected mice trended lower in spleens compared mice infected with WT Pb NK65, and was significantly less than levels found in livers of WT infected mice 
(Fig. 20B and C). On day 9 post Pb NK65 infection (day 2 post Lm), spleens and livers were removed, and bacterial burdens were analyzed. Similar to what was observed in $\mathrm{Py}+\mathrm{Lm}$, mice coinfected with $\mathrm{Pb}$ and $\mathrm{Lm}$ had higher bacterial burdens in spleens and livers than mice infected with Lm alone. However, contrary to our hypothesis, Lm burdens in $\Delta \mathrm{pm} 4$-infected mice were higher than $\mathrm{Pb}$ wt-infected mice in spleens and trended higher than $\Delta$ dpap-infected mice (Fig. 20D and E). Based on the data gathered from these experiments, deficiency in $\mathrm{Hz}$ production in this coinfection model do not impair clearance of Lm and more work is required to produce a model system where a normal Plasmodium infection can occur with the absence of $\mathrm{Hz}$ deposition in tissues and innate immune cells. 
A

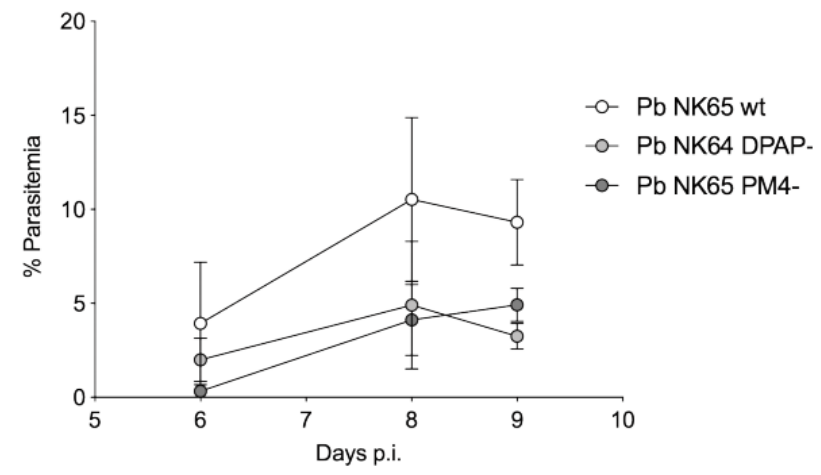

B

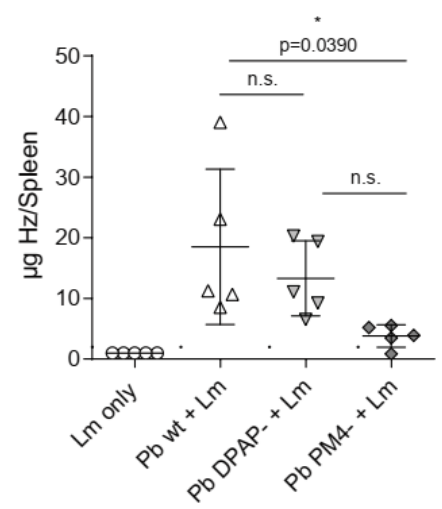

D

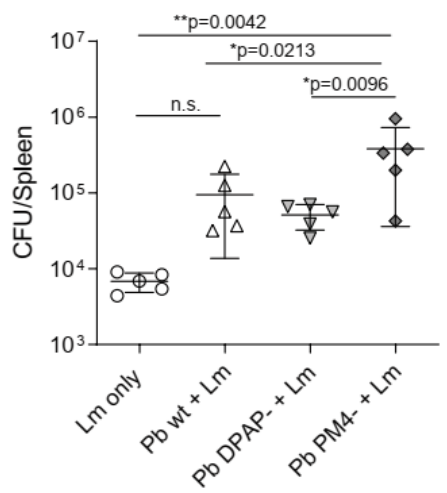

C

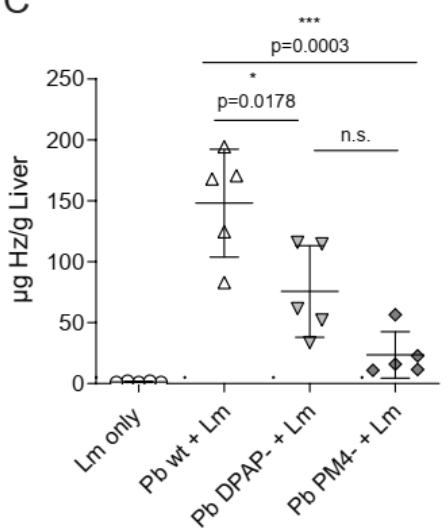

E

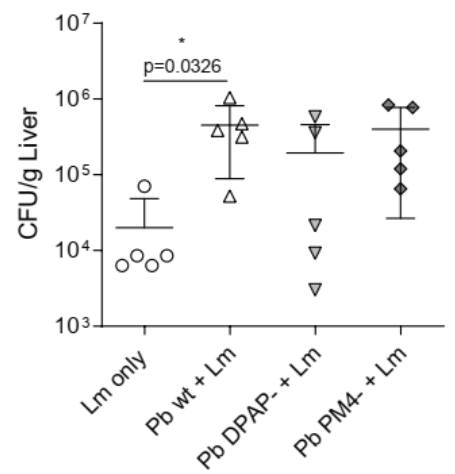

Figure 20. Infection with Plasmodium berghei mutant deficient in $\mathrm{Hz}$ does not delay $\Delta a c t A \mathbf{~ L m}$ clearance. C57BL/6 mice were given an intraperitoneal injection of $1 \times 10^{4} \mathrm{pRBCs}$ collected from donor mice infected with strains Pb NK65 (wt), Pb NK65 DPAP- (DPAP-), or Pb NK65 PM4- (Pm4-).

Parasitemia was analyzed on days 6,8 , and 9 post infection via flow cytometry (A). On day 7 , naïve control mice and groups of 5 mice were infected with each of the three $\mathrm{Pb}$ mutant strains were infected with $5 \times 10^{6} \mathrm{CFU}$ of AactA Lm. On day 9 post $\mathrm{Pb}$ infection (day 2 post $\mathrm{Lm}$ ), mice were sacrificed, and spleen and liver tissues were collected for both $\mathrm{Hz}$ quantification (B and C) and bacterial burdens (D and $\mathrm{E}$ ).

Data (mean \pm S.D.) are representative of a total of three independent experiments. Statistical significance was determined by one-way ANOVA followed by Tukey's multiple comparisons.

Although we did not observe defective ROS production in innate immune cells

treated with $\mathrm{Hz}$ in vitro or improved Lm clearance in spleens of mice infected with Hz-

deficient Plasmodium, our strong rationale to investigate the immunosuppressive activity

of $\mathrm{Hz}$ led us to investigate the effect $\mathrm{Hz}$ has on survival of bacteria within splenic 
phagocytes. Therefore, we used a gentamicin protection assay using sort-purified splenic phagocytes to test the hypothesis that in presence of $\mathrm{Hz}$, cells would have a defective ability to or control the intracellular growth of Lm. PyHz extracted from spleens and livers of mice that had cleared a Py infection was incubated with sort purified splenic phagocytes from naïve mice for 1-2 hours before cells were infected with Lm in a gentamycin protection assay, as described in Figure 13 in Chapter II. Following incubation with $\mathrm{PyHz}$, some $\mathrm{CD} 11 \mathrm{~b}^{+} \mathrm{CD} 11 \mathrm{c}^{-}$cells had phagocytosed $\mathrm{PyHz}$, while others did not (Fig. 21A). Consistent with the hypothesis, PyHz impaired the ability of

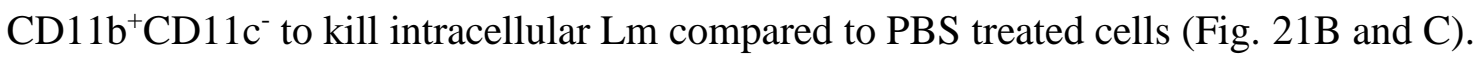
These data demonstrate that a parasite-derived factor $(\mathrm{PyHz})$ was capable of impairing the anti-bacterial effector mechanisms of $\mathrm{CD}_{11} \mathrm{~b}^{+} \mathrm{CD} 11 \mathrm{c}^{-}$cells in an in vitro assay. 
A 9 days post $\mathrm{PyHz}$ treatment
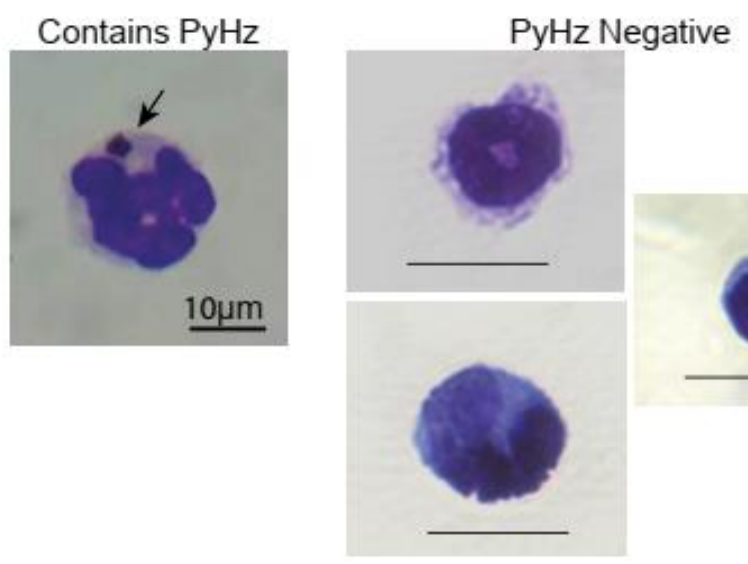

B

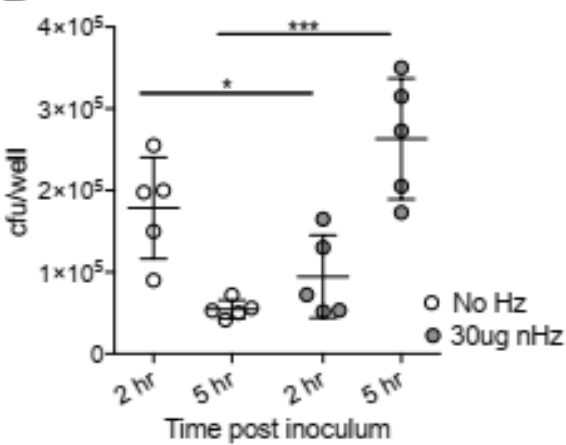

Whitney test.
Figure 21. P. yoelii -derived hemozoin and bound bioactive molecules impair anti-bacterial functions of splenic phagocytes. $10^{5}$ sort-purified CD11b ${ }^{\text {hi }} C D 11 c^{-}$splenic phagocytes were enriched, as in Figure 13 (A-B), from naïve mice and were incubated with $30 \mu \mathrm{g}$ hemozoin purified from spleen and livers of mice previously infected with Py (PyHz) for 1-2 hours. A) A giemsa-stained cell containing internalized $\mathrm{PyHz}$ (brown pigment), indicated by arrow, and $\mathrm{PyHz}$ negative cells containing no visible uptaken pigment. Intracellular $\triangle a c t A \mathrm{Lm}$ burden was determined after 2 hours and 5 hours (B) in a gentamicin protection assay to evaluate fold change at 5 hours post infection (C). Fold change in intracellular bacteria was determined by cfu/well at 5 hours post infection divided by cfu/well at 2 hours post infection. Data (mean \pm S.D.) are representative of 3 independent experiments each symbol represents an individual replicate of pooled cells from 3 mouse spleens per group. Statistical analysis was determined with Mann-

In vivo analysis of $\mathrm{Hz}$-induced suppression of innate immunity during bacterial infection

To test whether PyHz was able to impair the clearance of $\mathrm{Lm}$ in vivo, naïve mice were given intravenous injections of $\mathrm{PyHz}$ followed by $\Delta$ actA $\mathrm{Lm}$ infection 7-days later. PyHz was observed in some, but not all circulating leukocytes both 1- and 7-days post injection as well as in spleen and liver cells 9-days after injection (Fig. 22A). PyHz could also be quantified in both the spleen and liver 9-days post $\mathrm{PyHz}$ injection (Fig. 22B).

When Lm burden was quantified, $\mathrm{PyHz}$ treated mice had an elevated burden in the spleen (Fig 22C). With this data, we have shown that a parasite factor alone, $\mathrm{PyHz}$, is capable of impairing innate immune response to a secondary bacterial infection. 

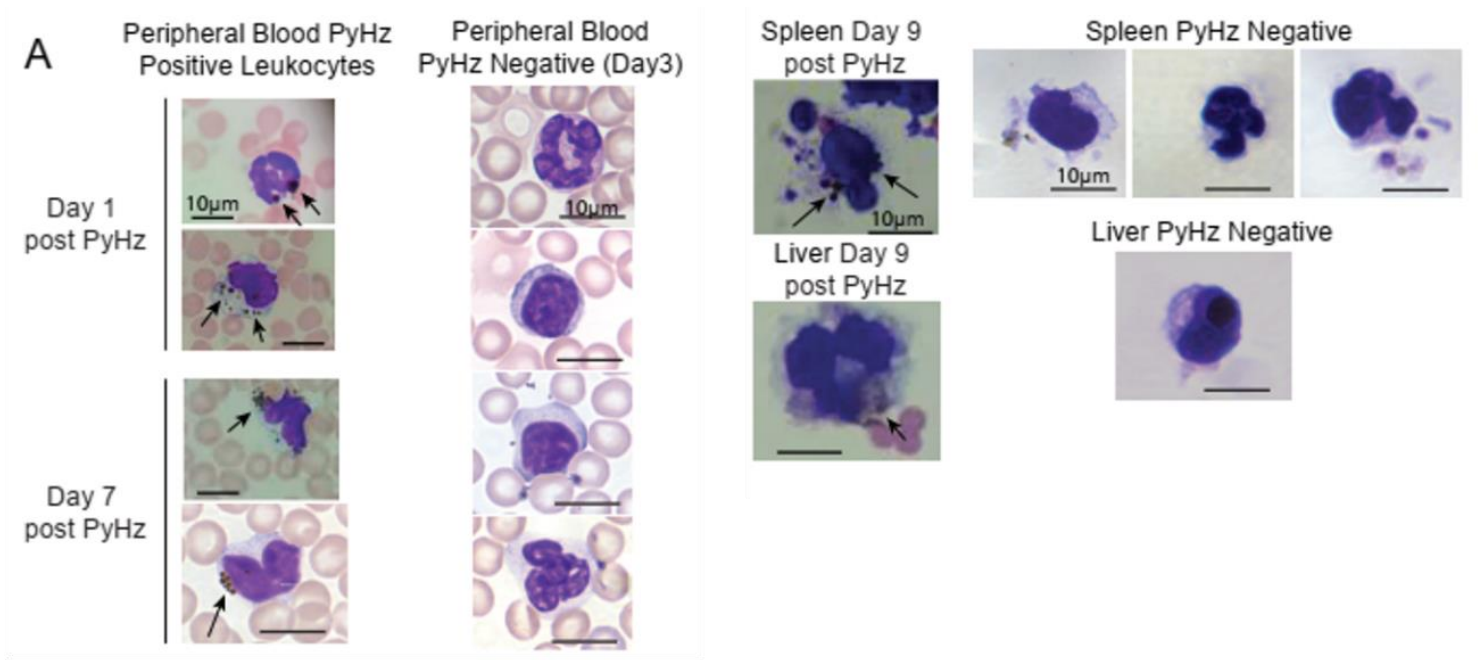

B
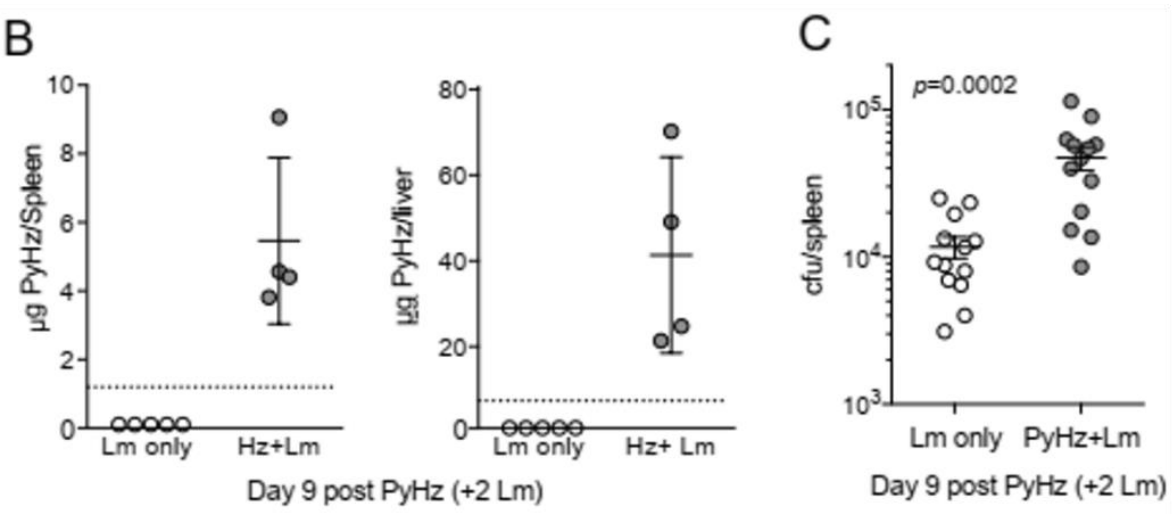

Figure 22. $P$. yoelii hemozoin with bound bioactive molecules is sufficient to impair clearance of $\triangle a c t A \mathbf{~ L m}$ in vivo. C57BL/6N mice treated i.v. with $1 \mathrm{mg} \mathrm{PyHz}$ isolated from livers and spleens of Py infected mice (A-C). 7 days after $\mathrm{Hz}$ treatment mice were infected with $5 \times 10^{6} \mathrm{CFUs} \triangle a c t A \mathrm{Lm}$. A ) Giemsa stained peripheral blood smears and cells mounted from spleen and liver single cell suspensions on the indicated days post $\mathrm{Hz}$ treatment. Arrows indicate the presence of intracellular $\mathrm{Hz}$, visible as brown pigment. $\mathrm{PyHz}$ and $\mathrm{PfHz}$-negative leukocytes were present at indicated time points in peripheral blood, spleen or liver with no visible intracellular $\mathrm{Hz}$ pigment (B and E) Quantification of $\mathrm{Hz}$ in spleens and livers. Data (mean \pm S.D.) are from a single experiment representing three independent experiments. Dotted lines represent the limit of $\mathrm{Hz}$ detection. Bacterial burden in spleens of $\mathrm{PyHz}(\mathrm{C}) \mathrm{PfHz}(\mathrm{F})$ or untreated control mice was determined 2 days post $\mathrm{Lm}$ infection. Data (mean \pm S.E.) are cumulative results from 2-3 independent experiments. Each symbol represents an individual mouse (G-I) C57BL/6N mice were treated i.v. with $1-1.5 \mathrm{mg}$ PyHz followed by i.n. infection with $10^{6} \mathrm{CFU}$ Sp 7 days later. Mice were sacrificed 2 days after Sp infection and lungs, blood, and spleens were collected for bacterial burden analysis. Data (mean \pm S.E.) are pooled results from 3 independent experiments. For all data, each symbol represents an individual mouse. Statistical significance was determined with Mann-Whitney test. 
Py is a rodent-specific species of Plasmodium, and previous studies exploring immune modulatory effects of $\mathrm{Hz}$ have typically isolated $\mathrm{Hz}$ from $P$. falciparum infected RBC cultures, rather than from organs of infected animal. To address potential differences in isolating $\mathrm{Hz}$ from organs of previously infected mice, and the ability of clinically relevant $P$. falciparum $\mathrm{Hz}$ to suppress innate immunity during bacteremia, $\mathrm{Hz}$ from P. falciparum infected red blood cell cultures (PfHz) was also tested. Consistent with $\mathrm{Hz}$ from $\mathrm{Py}$ infected mice, $\mathrm{PfHz}$ could be detected within some leukocytes in peripheral blood following injection, while others remained PfHz-negative (Fig. 23A), was quantified in both the spleen and liver (Fig. 23B), and PfHz treated mice had elevated bacterial burden two-days post Lm infection (Fig. 23C). 

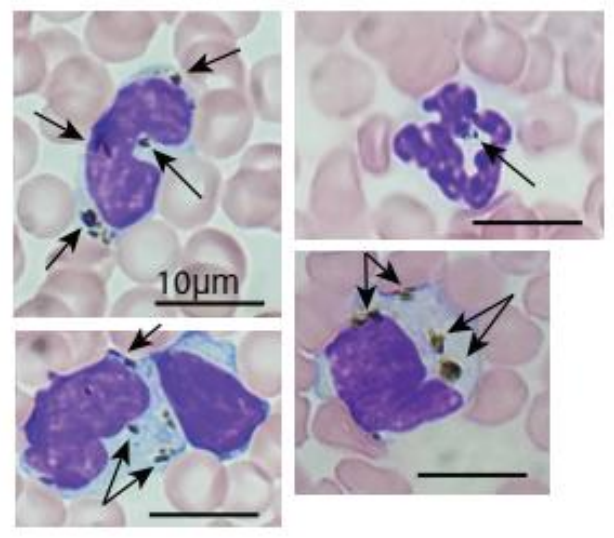

\section{Day 7 post $\mathrm{PfHz}$}

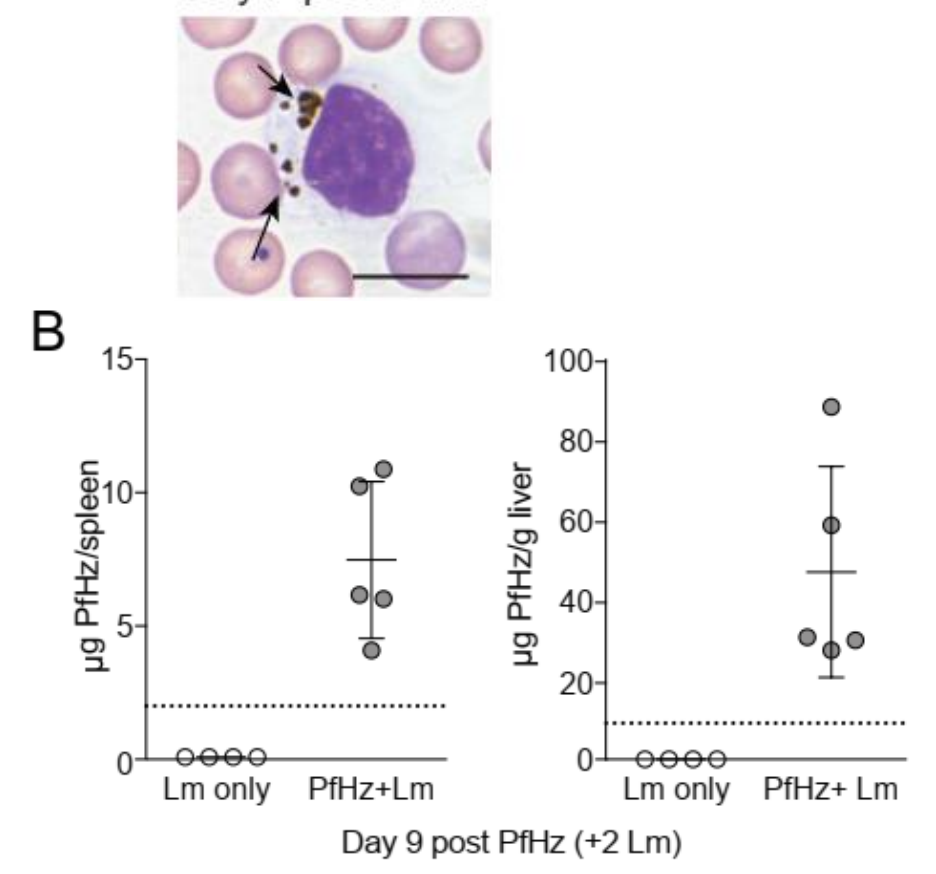

B

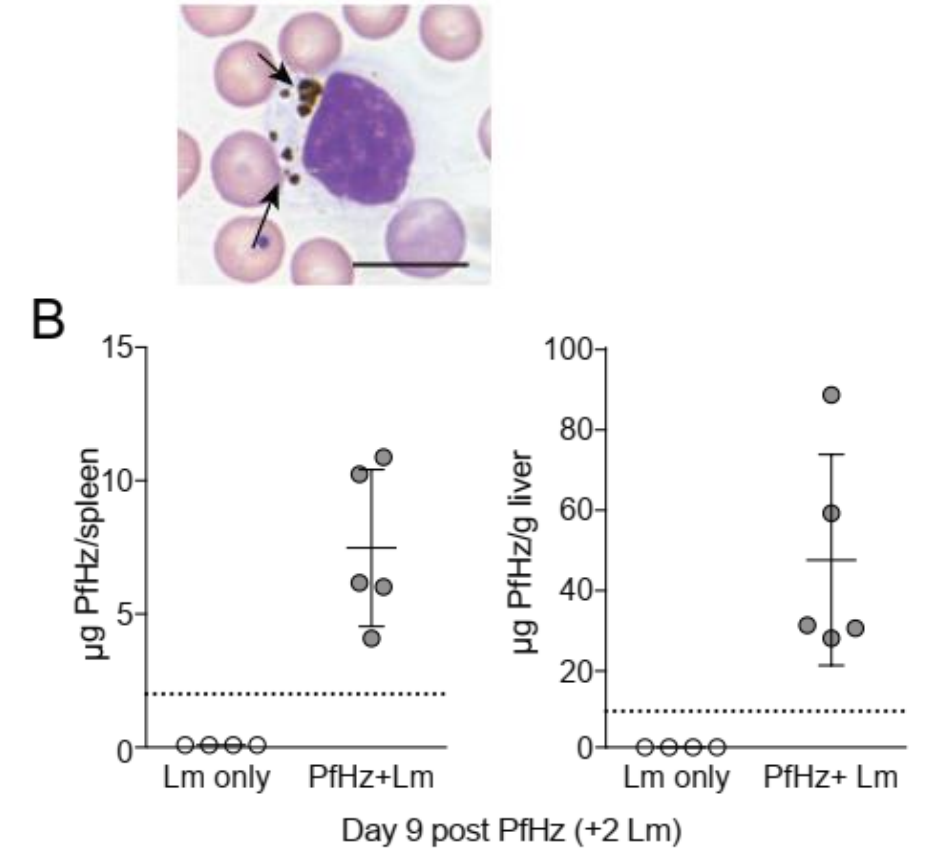

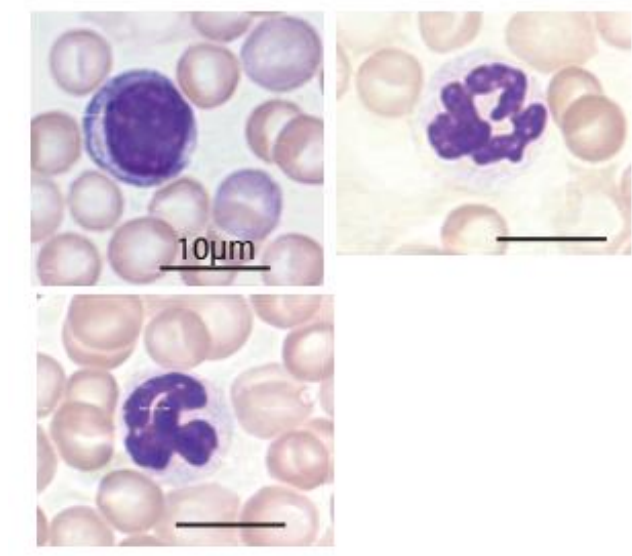

Peripheral blood $\mathrm{PfHz}$ Negative (Day3)

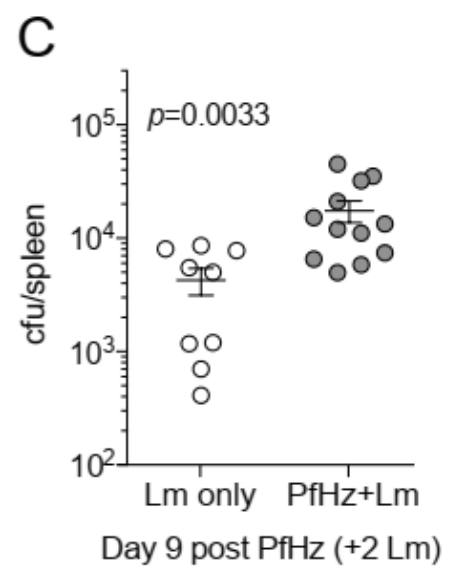

Figure 23. Plasmodium falciparum hemozoin ( $\mathrm{PfHz})$ is sufficient to impair clearance of $\triangle$ actA $\mathbf{L m}$ in vivo. C57BL/6N mice treated i.v. with $1 \mathrm{mg}$ hemozoin isolated from $P$. falciparum $(\mathrm{Pf})$ infected red blood cell culture (PfHz). 7 days after PfHz treatment mice were infected with $5 \times 10^{6} \mathrm{CFUs} \Delta a c t A \mathrm{Lm}$. A) Giemsa stained peripheral blood smears and cells mounted from spleen and liver single cell suspensions on the indicated days post $\mathrm{Hz}$ treatment. Arrows indicate the presence of intracellular $\mathrm{Hz}$, visible as brown pigment. PfHz-negative leukocytes were present at indicated time points in peripheral blood, spleen or liver with no visible intracellular $\mathrm{Hz}$ pigment (B) Quantification of $\mathrm{Hz}$ in spleens and livers. Data (mean \pm S.D.) are from a single experiment representing three independent experiments. Dotted lines represent the limit of $\mathrm{Hz}$ detection. Bacterial burden in spleens of $\mathrm{PfHz}(\mathrm{C})$ or untreated control mice was determined 2 days post Lm infection. Data (mean \pm S.E.) are cumulative results from 2-3 independent experiments. Each symbol represents an individual mouse. 
Research concerning the immunomodulatory effects of hemozoin has been conducted in a large variety of preparations, relating to drastic differences in molecular components associated with the biocrystals, which confer dissimilar results. Thus, we performed a series of experiments which aimed to explore how different hemozoin preparation procedures, and therefore, surface-associated bioactive molecules impact the clearance of $\mathrm{Lm}$. PfHz was treated with DNase to remove DNA whereas $\mathrm{PyHz}$ had not been DNase treated. When PyHz was treated with DNase to remove DNA, mice injected with DNA free PyHz still exhibited elevated bacterial burden (Fig. 24A-C). In contrast to these results, mice treated with 'clean' PyHz, which was treated with detergents and proteinase K, followed by infection with $\mathrm{Lm}$, showed no defect in the control of $\mathrm{Lm}$ (Fig. 24D). Similarly, mice that were treated with pure synthetic $\mathrm{Hz}$ followed by Lm infection, also showed no defect in control of Lm (Fig. 24E). These results support the conclusion that the bioactive molecules associated with hemozoin (other than DNA) are involved in the immunomodulatory properties that contribute to delayed Lm clearance in our model. 
A
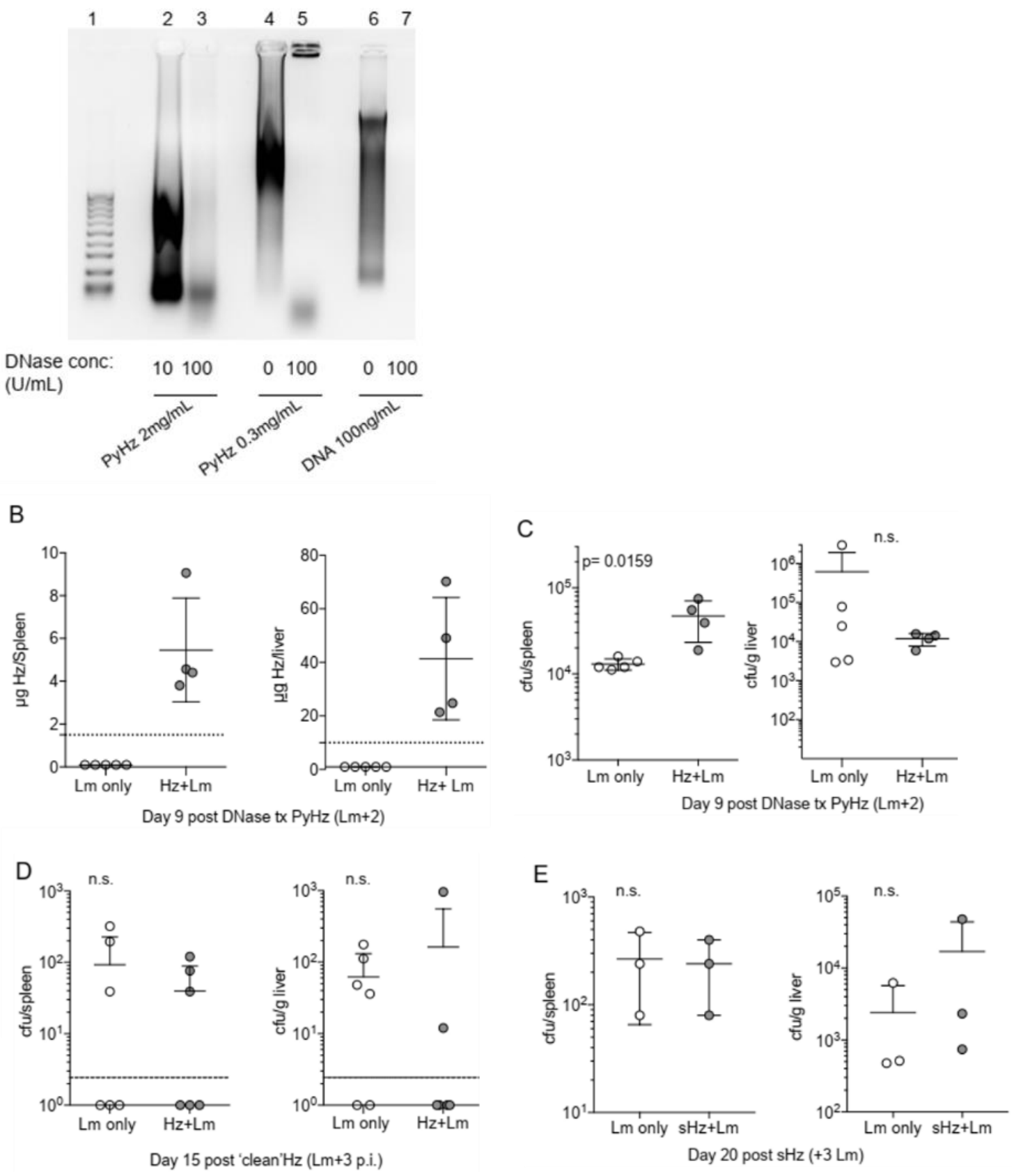

Figure 24. Hemozoin preperation differentially impacts anti-bacterial innate immunity. A) Agarose gel containing PyHz samples labeled with GelRed Nucleic Acid stain to detect presence of DNA. Lane 1 $1,000-100$ base pair ladder, lane $2-15 \mu \mathrm{L}$ sample of $2 \mathrm{mg} / \mathrm{mL}$ PyHz treated with $10 \mathrm{U} / \mathrm{mL}$ DNase, lane 3 $15 \mu \mathrm{L}$ sample of $2 \mathrm{mg} / \mathrm{mL} \mathrm{PyHz}$ treated with $100 \mathrm{U} / \mathrm{mL}$ DNase, lane $4-15 \mu \mathrm{L}$ sample of untreated 0.3 $\mathrm{mg} / \mathrm{mL}$ PyHz, lane $5-15 \mu \mathrm{L}$ sample of $0.3 \mathrm{mg} / \mathrm{mL}$ PyHz treated with $100 \mathrm{U} / \mathrm{mL}$ DNase, lane $6-15 \mu \mathrm{L}$ of $100 \mathrm{ng} / \mathrm{mL}$ untreated DNA, lane $7-15 \mu \mathrm{L}$ of $100 \mathrm{ng} / \mathrm{mL}$ DNA treated with $100 \mathrm{U} / \mathrm{mL}$ DNase. B) Quantification of DNase treated PyHz in spleens and livers. Data (mean \pm S.D.) are representative of three independent experiments. Dotted lines represent the limit of $\mathrm{Hz}$ detection. C-E) Lm burdens in spleens and livers from mice treated with DNase treated $\mathrm{Hz}(\mathrm{C})$, 'clean' $\mathrm{PyHz}(\mathrm{D})$, and synthetic $\mathrm{Hz}(\mathrm{E})$ on the indicated days post $\mathrm{Hz}$ treatment and $\mathrm{Lm}$ infection. Data were analyzed by Mann Whitney test. 
So far, we have characterized the contribution of $\mathrm{Hz}$ to both the delayed clearance of $\mathrm{Lm}$ in our Py+Lm coinfection model and demonstrated that $\mathrm{Hz}$ impairs antibacterial functions of splenic phagocytes. Our use of the model bacterium, Lm, in our coinfection model provided critical insight into how Plasmodium infection weakens host immunity to invasive bacterial infections, we next sought to test whether hemozoin has a similar impact on the more clinically relevant pneumococcal coinfection model. Similar to experiments in figures 18-20, naïve mice were given intravenous injections of $\mathrm{PyHz}$ followed by intranasal infection with $\mathrm{Sp} 7$ days later. Unlike what was observed in $\mathrm{Lm}$ infections, $\mathrm{PyHz}$ did not show different bacterial burdens in the lungs, peripheral blood, or spleen compared to control mice (Fig. 25A-C). While this in vivo data does not support a role for $\mathrm{Hz}$ in Plasmodium-induced susceptibility to invasive Sp infection, it does not rule out the possibility. More work is needed to test this hypothesis further. Collectively, the results of this chapter identify $\mathrm{Hz}$ with bound bioactive molecules, excluding DNA, as a parasite-derived factor that is able to impair anti-bacterial innate immunity to some bacterial pathogens. 

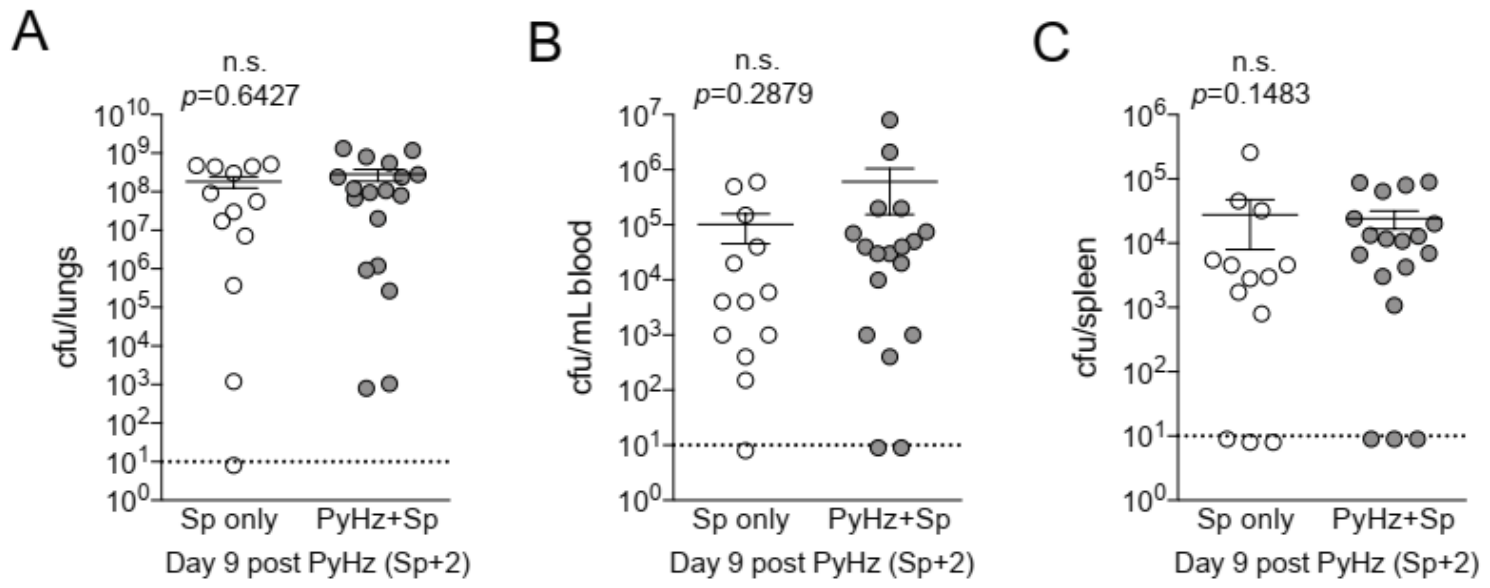

Figure 25. P. yoelii hemozoin with bound bioactive molecules does impair immunity to Sp. C57BL/6N mice were treated i.v. with $1-1.5 \mathrm{mg} \mathrm{PyHz}$ followed by i.n. infection with $10^{6} \mathrm{CFU} \mathrm{Sp} 7$ days later. Mice were sacrificed 2 days after Sp infection and lungs, blood, and spleens were collected for bacterial burden analysis. Data (mean \pm S.E.) are pooled results from 3 independent experiments. For all data, each symbol represents an individual mouse. Statistical significance was determined with Mann-Whitney test.

\section{Discussion}

It has been postulated that Plasmodium-induced immune suppression and susceptibility to invasive bacterial infections is not attributed to a parasite-specific factor. Rather, it is due to Plasmodium-induced hemolysis, and subsequent activation of HO-1 and IL-10 that inhibit neutrophil functions lead to weakened immune responses to invasive bacterial infections [60]. Yet, we demonstrate that Py-induced immune suppression lasts up to 2-months post clearance of Py, when the effects of hemolysis are likely no longer in play. Additionally, Cunnington and colleagues demonstrated that neutrophils collected from patients previously infected with $P$. falciparum had defective ROS burst following PMA stimulation that lasted up to 8 weeks-post treatment [61]. Of note, $\mathrm{Hz}$ from $P$. berghei infection remains deposited in liver, bone marrow, and spleen tissues as well as within isolated $\mathrm{CD} 11 \mathrm{~b}^{+}$cells isolated from those tissues and peripheral blood of mice for at least 196 days post infection [110]. Collectively, these data provide 
evidence that a parasite factor, $\mathrm{Hz}$, may contribute to this impaired innate immunity to bacterial coinfections and leads to the hypothesis that this factor contributes to clinical observations of reduced immunity to invasive bacterial infections in patients with history of malaria.

Using a murine infection model of malaria and ex vivo intracellular bacterial survival assays we have determined that $\mathrm{Hz}$ is able to impair innate immunity to bacterial infections independent of the parasite infection. Of note, we made this observation using two different preparations of $\mathrm{Hz}$. First, $\mathrm{Hz}$ was prepared via a novel method by extracting $\mathrm{Hz}$ from organs of mice previously infected with Py. Importantly, DNase treated PyHz, to eliminate parasite and mouse DNA, was still capable of suppressing innate immunity to Lm in vivo (Fig. 24 A-C). Second, we demonstrated natural $\mathrm{PfHz}$ prepared from infected red blood cell cultures [84] was able to suppress innate immunity in vivo. These data demonstrate that the immune modulatory effect of $\mathrm{PyHz}$ is unlikely due to parasite or mouse DNA. Whereas parasite or mouse DNA were not implicated in the immune suppressive nature of $\mathrm{PyHz}, \mathrm{PyHz}$ prepared in the presence of detergents and proteases (Fig. 24 D), as well as synthetic $\mathrm{Hz}$ (Fig. $24 \mathrm{E}$ ) did not impair in vivo innate immune responses. This supports the idea demonstrated by Carney et al. that the immune modulatory effect of $\mathrm{Hz}$ is perhaps not due to $\mathrm{Hz}$ itself or adherent DNA, but attributed to interactions with RBC membrane lipids and other debris that subsequently generate highly reactive secondary metabolic products capable of disrupting cell functions (e.g. NADPH oxidase and iNOS) upon uptake [185]. Furthermore, lipids within parasitized RBCs and hemozoin, such as the hydroxy fatty acids, hydroxy-arachidonic acid (HETE) 
and hydroxy-linoleic acid (HODE), have been shown to inhibit PMA-stimulated reactive oxygen burst in human peripheral blood monocytes [186].

Our results suggest the possibility that parasite-derived $\mathrm{Hz}$ and bound molecules are contributing to the impaired innate immune response to Lm, potentially through inhibition of ROS production. While our data indicate that $\mathrm{Hz}$ impairs immunity to $\mathrm{Lm}$, our 'native' preparations of PyHz did not impair immunity to $\mathrm{Sp}$ in our model system. There are two potential explanations for this observation. First, intravenous injection of $\mathrm{PyHz}$ may not have resulted in sufficient levels of $\mathrm{Hz}$ to suppress innate immunity to $\mathrm{Sp}$. This possibility is supported by the observation that Py infection impairs innate immunity to Sp long after the clearance of Py (Fig. 9D-F), suggesting a bona fide role for $\mathrm{Hz}$ in this immune suppression. Second, differences in Hz-driven impaired innate immunity between Lm and Sp may be explained by the intracellular and extracellular nature of theses pathogens, respectively. Lm is considered an "intracellular pathogen" since it grows within cells, whereas $\mathrm{Sp}$ is generally thought of as extracellular. Although this is not exclusive as $\mathrm{Sp}$ can grow in $\mathrm{CD} 169^{+}$splenic macrophages [187].

A previous study found that digestive vacuoles isolated from $P$. falciparuminfected human red blood cell culture are rapidly phagocytosed by neutrophils in a complement-mediated manner [161]. This same group also showed that uptake of $\mathrm{Hz}$ loaded digestive vacuoles induced a ROS burst when opsonized with active serum, but both uptake and ROS burst did not occur if digestive vacuole membrane was disrupted by either ultrasonication or detergent treatment [162]. Another interesting finding was that internalizing digestive vacuoles by PMNs reduced ROS production in response to infection with Staphylococcus aureus and also diminished bacterial killing. In agreement 
with the work of Dasari et. al, our purified $\mathrm{PyHz}$ and $\mathrm{PfHz}$, which is freed from digestive vacuoles, did not impair ROS production of either splenic neutrophils in whole spleen single cell suspension or purified bone marrow neutrophils in response to PMA, Lm, or Sp (Figure 18 and 19). Contrary to these studies, however, we were able to visualize uptake of PyHz by sort-purified splenic CD11b ${ }^{+} / \mathrm{CD}^{11 c^{-}}$phagocytes (Figure 21A) and found that PyHz-treatment reduced the ability of these cells to control intracellular growth of Lm (Figure 21B and C).

When conducting our in vitro analysis of ROS production of $\mathrm{PyHz}$ treated whole spleen single cell suspensions and purified bone marrow neutrophils, we observed massive quantities of intracellular ROS being produced in cells infected with live opsonized Sp, nearly 100-fold higher than uninfected cells and close to 10-fold higher than heat-killed opsonized Sp or Lm infected cells (Figures 18 and 19). Furthermore, PyHz treated splenic PMNs and cells infected with live opsonized-Sp had significantly higher levels of intracellular ROS than cells with no $\mathrm{Hz}$ treatment. As mentioned in Chapter III, Sp is resistant to ROS produced by PMNs and produce $\mathrm{H}_{2} \mathrm{O}_{2}$ itself [170]. This result could be interpreted as an indirect detection of metabolically active intracellular Sp, through the production of ROS, and indicate that there is a greater amount of unkilled $\mathrm{Sp}$ in cells that were treated with PyHz. Further experiments are needed to determine if there is a Hz-dependent defect in the ability of PMNs to kill both extracellular and intracellular Sp. Since it has been discovered that $\mathrm{Sp}$ is able to grow intracellularly within some subsets of splenic macrophages [187], it would also be interesting to explore the possibility that following $\mathrm{Hz}$ phagocytosis, $\mathrm{Sp}$ is able to grow within some leukocytes. 
Despite the results in experiments with $P$. berghei mutants that did not support the hypothesis that $\mathrm{Hz}$ contributes to delayed clearance of Lm in vivo (Figure 20), modifications to the coinfection model could be made to improve the system in order to better assess this question. One possible reason for the lack of a Hz-dependent phenotype in this model is the relatively low parasitemia and the kinetics of Pb NK65 infection in mice. In Py infection, parasites begin to replicate at detectable levels in the blood around 5 days post infection and by day 9 parasitemia reaches 10 to $15 \%$. Mice go on to develop acute hyperparasitemia that plateaus between 15 - and 20-days post infection around $60 \%$ (Fig. 4A). The progression of $\mathrm{Pb}$ NK65 is quite different, with parasitemia increasing from approximately $1 \%$ on day 5 to $\sim 8 \%$ on day 9 followed by a continued increase [188]. During the early acute Pb NK65 parasitemia, lung weights begin increasing due to hemozoin-induced acute lung injury and mice eventually succumb to malaria-associated acute respiratory distress syndrome (MA-ARDS) around day 12 post infection without complications from cerebral malaria [81, 177]. These differences may contribute to the lack of impaired antibacterial immunity observed in the $\mathrm{Pb}+\mathrm{Lm}$ coinfections. Another complication preventing a definitive conclusion that $\mathrm{Hz}$ does not impair clearance of $\mathrm{Lm}$ is that there was not a significant difference between $\mathrm{Hz}$ quantities of liver and spleens of $\mathrm{Pb} \Delta p m 4$ (Hz-deficient) infected mice and $\mathrm{Pb} \Delta d p a p$ infected mice which serve as a control for the attenuated parasitemia of $\mathrm{Pb} \Delta p m 4$ (Figure $20 \mathrm{~B}$ and $\mathrm{C}$ ). To improve this model it will be necessary to compare levels of $\mathrm{Hz}$ in spleens from Py-infected mice to the three $\mathrm{Pb}$ strains used here at day 7 and 9 post infection to determine if $\mathrm{Pb}$ infected mice had lower levels of $\mathrm{Hz}$ that may have impacted Lm clearance. 
Our in vitro data demonstrates the impaired anti-bacterial ability of splenic phagocytes either isolated from Py-infected mice or treated with $\mathrm{PyHz}$. Interestingly, the analysis of intracellular cfu burdens in our in vitro assay found that splenic phagocytes from Py-infected mice, or phagocytes treated with $\mathrm{PyHz}$, contained significantly less Lm at 2 hours post infection (with 1 hour in gentamicin) compared to cells from naïve mice or untreated cells (Fig. 16C and F). There are several explanations for this result, including a defect in Lm uptake, differences in Lm adherence, and altered killing of intracellular or extracellular Lm during the time prior to the 2-hour time point. Additional work will be needed to further understand the impact of Py-infection and hemozoin in these interactions. Furthermore, cfu counts at 5 hours post infection suggest that $\mathrm{PyHz}$ treated or Py-infected mouse phagocytes exhibit a profound inability to restrict intracellular bacterial growth. In contrast, there may be a different mechanism responsible for Plasmodium-dependent susceptibility to extracellular bacteria. For example, serum-dependent effects as we observed (Fig. 15C). Further studies are required to elucidate the mechanisms underlying susceptibility to Sp and other extracellular bacterial pathogens during coinfection with Plasmodium. Methods that can recognize the presence or absence of internalized $\mathrm{Hz}$ at the single cell level would substantially enhance our understanding of this interaction. Additional work is also needed to uncover the immune cell types affected as well as the antimicrobial functions that are impaired by Plasmodium in the context of Sp and other clinically important coinfections during malaria.

$\mathrm{Hz}$ may directly impair antibacterial effector mechanisms of innate immune cells via uptake or interaction with $\mathrm{Hz}$. Alternatively, the uptake and recognition of $\mathrm{Hz}$ by 
phagocytic cells could have an indirect effect through altering the host microenvironment via the induction of pathways that influence the production of cytokines and chemokines in a manner that creates a more favorable environment for invasive/pathogenic bacteria. Additionally, $\mathrm{Hz}$ has been shown to induce trained immunity [112], suggesting the possibility that long-term suppression of innate immunity to systemic bacterial infections may in part be attributed $\mathrm{Hz}$-induced epigenetic modifications.

Clinical reports of invasive bacterial disease coinciding with malaria often report both NTS and Sp as the two organisms most commonly isolated from blood.

Experimental studies to date have focused almost exclusively on NTS and it is unknown whether the mechanisms of Plasmodium-induced immune suppression to NTS will be important for Sp or other bacterial pathogens found in coinfections with malaria (e.g., Haemophilus influenzae, Mycobacterium tuberculosis, Staphylococcus aureus, Streptococcus pyogenes, etc. [42-49, 54]). We report for the first time, to our knowledge, a murine Plasmodium and pneumococcal coinfection. Mice were infected with $P$. yoelii 17XNL followed by a $S$. pneumoniae ATCC \#6303, a type 3 capsule serotype strain, via the natural intranasal route. Consistent with the risk of invasive Sp infections in humans with malaria [42], we found decreased survival and increased bacterial burdens in coinfected mice compared to control Sp only infected mice. Murine models of malaria associated-respiratory distress syndrome (MA-ARDS) using P. berghei NK65 strain have found an association between pulmonary pathology and the levels of $\mathrm{Hz}$ deposition [81]. Pulmonary pathology matching MA-ARDS has been reported in Py 17X but not Py 17XNL infection [189]; however, the parasitemia obtained in mice infected with Py $17 \mathrm{XNL}$ in that study were much lower than what was observed in this report where Py 
17XNL parasitemia reached 40-60\%. Consequently, $\mathrm{Hz}$ deposition in the lung, and potentially in spleen and other organs, may contribute to differential invasiveness of $\mathrm{Sp}$ during Plasmodium infection. Further studies are necessary to determine to what degree Plasmodium, via $\mathrm{Hz}$ or other mechanisms, induces lung pathology and to what degree this contributes to increased susceptibility to invasive Sp infection. 


\section{CHAPTER V \\ FINAL DISCUSSION AND FUTURE DIRECTIONS}

In this work, we have characterized the impact of Plasmodium infection on immunity to 3 different bacterial pathogens using three murine models of malaria bacteria coinfections, and a series of in vitro assays. Our use of Listeria monocytogenes as a model bacterium to probe antimicrobial immune responses led us to the findings that $P$. yoelii infection delays clearance of bacteria from the spleen in a model of non-lethal systemic infection using and attenuated strain of Lm. Further investigations led us to discover that the impaired innate immune response associated with Py infection lasts long after clearance of patent (i.e. detectable), Py infection, and this Py-driven immunosuppression occurs independent of IFN $\gamma$ or the hemolysis-dependent mechanisms that have been attributed to the well-characterized increased susceptibility to invasive Salmonella disease (HO-1, and IL-10). Perhaps most importantly, this work demonstrates for the first time, that hemozoin, a parasite factor, contributes to impaired immunity to secondary bacterial infection. These findings are summarized in Figure 28. Hemozoin had previously been shown to have many effects on in vitro cultured cells, yet, inconsistency in source, purification, and preparation techniques have resulted in different camps regarding whether hemozoin is immunomodulatory at all or which of the surface-bound biomolecules are relevant. This work has contributed to the conversation by showing that $\mathrm{Hz}$ alone ("clean" preparation with detergents and protease 
treatment) did not impair immunity to Lm, whereas $\mathrm{Hz}$ containing bound-bioactive molecules is sufficient to impair clearance of bacteria from spleens of Lm infected mice. We also show in vitro that treating splenic phagocytes with $\mathrm{Hz}$ containing bioactive molecules decreased their ability to limit the intracellular growth of Lm.

While we did not provide direct evidence of an Hz-dependent defect in ROS production in vitro or enhanced susceptibility to invasive Pneumococcal disease, we have two pieces of data that support a role for $\mathrm{Hz}$ in the impaired immunity to Sp. First, our observation that Py infected mice are more susceptible to invasive Sp infection not only during acute parasitemia, but months after clearance of detectable parasites from the blood stream, suggests that $\mathrm{Hz}$ is likely involved due to its long-term deposition in tissues with known importance in immune responses and active clearance of invasive Sp infection [81]. Second, we found an influx of intracellular ROS in PMNs from whole spleen single cell suspensions or BMNs isolated from naïve mice when they were treated with PyHz and subsequently infected with live opsonized-Sp compared to controls with no $\mathrm{Hz}$ treatment. We interpreted this result as potential indirect evidence of greater amounts of live, metabolically active $\mathrm{Sp}$ in Hz-treated cells. In fact, Sp can produce large quantities of $\mathrm{H}_{2} \mathrm{O}_{2}$, and several studies have provided evidence that $\mathrm{Sp}$ is not susceptible to killing via NADPH oxidase-dependent mechanisms, such as experiments using NADPH oxidase inhibitors, and the fact that PMNs isolated from patients with chronic granulomatous disease are not defective in killing Sp [190, 191]. Thus, high levels of ROS in Hz-treated PMNs infected with live-opsonized Sp compared to cells with no Hz treatment could be indirect evidence supporting the hypothesis that $\mathrm{Hz}$ treatment impairs the ability to kill $\mathrm{Sp}$ intracellularly. It is known that $\mathrm{Sp}$ is resistant to oxidative stress, 
such as ROS produced by PMNs, and is killed by PMN serine proteases. Both cathepsin $\mathrm{G}$ and elastase are capable of killing $\mathrm{Sp}$, since the individual inhibition of either did not decrease Sp killing in an opsonophagocytic assay, yet treatment with a protease inhibitor eliminated killing [172]. Thus, there is apparent redundancy in the capacity of neutrophil proteases to sufficiently kill Sp in the context of complement-dependent opsonophagocytosis and serine proteases appear to be the main factors in Sp killing. More work is required to investigate the hypotheses that the ROS we observed in $\mathrm{Hz}$ treated PMNs was produced by viable, metabolically active Sp rather than from the PMNs intracellular NADPH oxidase-dependent ROS production and that there is indeed an Hz-dependent defect in killing of Sp. The experiments that aim to explore the role of $\mathrm{Hz}$ in enhanced susceptibility to Sp are highlighted in the future directions section in this chapter.

Considering whether neutrophils acquire their defective antibacterial functions in the bone marrow or after entering a tissue will be important in pursuing the mechanisms that lead to neutrophil disfunction in Plasmodium infections. By isolating neutrophils from bone marrow of Py-infected and naïve mice and performing experiments testing their antibacterial functions in vitro, it would be possible to compare with peripheral blood and tissue-isolated neutrophils to determine the origin of the defect. While data from Hz-treated PMNs isolated from bone marrow of naïve mice did not demonstrate a role for $\mathrm{Hz}$ in Plasmodium-induced impaired ROS, other antibacterial functions could be tested in this setting.

A key component of neutrophils arsenal against invading microbes is their ability to exocytose granules that contain toxic products. Of the four granules, gelatinase, 
specific, and azurophilic types are antibacterial, while secretory vesicles are not [192]. Release of azurophilic, specific and gelatinase granules by naïve versus Py-infected mouse BMNs can be tested in response stimuli by quantifying the surface markers associated with each granule type using flow cytometry [193]. In these experiments, granule exocytosis can be tested in response to fMLF, TNF $\alpha$, or Sp with or without opsonization from Py and naïve mouse serum. Given that serum opsonization of bacterial pathogens can influence granule exocytosis [194], including different preparations of serum in these experiments could help determine the contribution of a defective serum component in Py-infected mice. Other neutrophil functions that would be interesting to test, comparing BMNs from Py-infected and naïve mice to tissuederived or peripheral blood-isolated PMNs from Py-infected and naïve mice, include phagocytic capacity, phagosome maturation and bacterial killing efficiency. Due to the relatively low number of PMNs that can be isolated from peripheral blood of mice, samples from several mice can be pooled together in obtain enough cells for the conditions needed in the assays, as done in Schneider et al. [195].

In addition to antimicrobial functions, the ability of neutrophils to migrate to infected tissues via chemotaxis could also be examined. Although greater numbers of neutrophils were seen in spleens of Py-infected mice, compared with naïve mice at each time point post Py that was examined (Figure 12C), the ability of neutrophils to migrate to the spleen in response to an invasive bacterial infection following Py-infection is unknown. A kinetic study of splenic myeloid cells similar to what was conducted in Chapter III figures 11-13 could be performed to investigate this, where both naïve and Py-infected mice would be infected with Lm or Sp and the quantities of neutrophils in 
spleens can be determined with the same method. An in vitro system could also investigate the chemotactic ability of neutrophils following Py infection by placing Pyinfected and naïve mouse BMNs, peripheral blood neutrophils, or splenic neutrophils in a transwell system and examine their movement across the membrane in response to fMLF, IL-8, or IL-12, which is known to be protective in Sp infections by promoting recruitment of neutrophils to lungs [196].

Since $\mathrm{Hz}$ is known to be deposited in bone marrow for up to 140 days post $P$. chabaudi infection and is found in $\mathrm{CD}_{11} \mathrm{~b}^{+}$bone marrow cells over 280 days post $P$. berghei [67], any Hz-dependent mechanism influencing the antibacterial functions of neutrophils would detectable in an experiment with cells isolated from bone marrow of Py-infected mice long after parasite clearance. Thus, the same neutrophil functional responses mentioned above could be examined at timepoints during acute parasitemia at 15 days post-Py, after clearance of Py infection at day 30 post-Py, two months after Py clearance on day 100 post-Py, and even longer to determine the full kinetics and longevity of impaired neutrophil functions following Plasmodium infection.

Another important contribution this work adds to the study of bacterial coinfections in malaria is the first murine model of malaria and Pneumococcus coinfection. This model provides an essential tool to study the mechanisms contributing to the enhanced susceptibility to invasive Pneumococcal disease that has been described in patients with malaria in several clinical reports $[42,45,47,49,52-54,75,76,87]$. In characterizing this model, we found that Py infected mice had decreased survival and higher bacterial burdens in peripheral blood and spleens compared to mice infected with Sp alone 3 days after Sp infection. This impaired immunity to Sp in coinfected mice was 
apparent during acute malaria and 2 months after clearance of detectable parasites in the bloodstream. Finally, our results indicated that the activity of Py-induced IL-10 contributes to the increased susceptibility to $\mathrm{Sp}$ in coinfected mice, similar to what has been shown in NTS coinfections [59].

Together, our work reveals that Plasmodium infection induces long-term immunosuppression that renders the host susceptible to invasive bacterial coinfections that lasts beyond resolution of the parasite infection. Moreover, the data presented here, in agreement with clinical observations connecting malaria with increased severity of invasive bacterial disease, indicate the importance of obtaining information beyond a blood smear in order to account for not only active cases of malaria, but any recent history of Plasmodium infection. With the murine coinfection models, and in vitro assays described here to test Plasmodium-induced immunosuppression, more discoveries can be made to direct efforts to develop treatments and provide further evidence to argue for the need of heightened clinical surveillance and perhaps intervention in patients with invasive bacterial disease.

\section{Additional directions for investigating Plasmodium-Pneumococcal coinfections}

The Py-driven susceptibility/impaired innate immunity to Sp uncovered within this work may be occurring by several potential mechanisms that were not examined experimentally and there is rationale to further pursue these possibilities. Although $\mathrm{Sp}$ is resistant to intra-phagolysosomal ROS [172, 197], the dramatic decrease in Sp-induced intracellular ROS response in splenic PMNs from Py-infected mice (Figure 15) suggests 
other PMN antimicrobial responses may also be impaired in Py-infected mice.

Experimental tools are available to investigate PMN antimicrobial responses, including inhibitors of specific PMN granules inhibitors of cell signaling pathways. These experiments combined with an assay that tests intracellular and extracellular survival of Sp in culture with splenic phagocytes from naïve and Py-infected mice, similar to the gentamicin protection assay, would help elucidate specific mechanisms underlying impaired immunity to $\mathrm{Sp}$.

Previous work has found that inhibition of NADPH oxidase does not affect killing of Sp by human PMNs in vitro, suggesting that $\mathrm{Sp}$ is not directly killed by PMN ROS production [172]. This does not necessarily mean that this defective function does not impair PMN antibacterial functions that contribute to Sp killing. The lack of PMN production of ROS seen in cells from Py-infected mice could affect other antimicrobial processes that are known to kill $\mathrm{Sp}$, such as the activation of pre-formed neutrophil proteases and myeloperoxidase catalysis, which require NADPH oxidase-dependent ROS $[172,198,199]$. ROS production is also needed to acidify the phagolysosome to assist this bactericidal process [152].

One serine protease produced in high amounts during release of azurophilic granules by PMNs, elastase, is known to cleave TLR2, TLR4, and other important surface proteins known to recognize Sp [166]. Additionally, the same study found that this PMN elastase-dependent activity reduced cellular immune responses following heattreated Sp stimulation and reduced survival in an intratracheal model of murine Sp infection [166]. Plasmodium infection elicits a release of extracellular serine proteases that have been attributed to collateral inflammatory damage during malaria disease [200]. 
Therefore, the serine proteases induced by Plasmodium infection may cleave important receptors from innate immune cells, rendering them unable to perform their antibacterial effector functions against a secondary Pneumococcal infection. This hypothesis could be tested in our murine model of $\mathrm{Py}+\mathrm{Sp}$ coinfection with either use of protease inhibitor treatment, or conditional knockout mice that lack the ability to produce these enzymes. Quantification of surface receptors impacted by protease cleavage could be performed with flow cytometry to verify the extent of the Plasmodium-elicited damage, and then correlate with a careful characterization of Sp burdens.

\section{Further experiments to explore whether differences in splenocyte populations between Py-infected and nä̈ve mice impacts antibacterial immunity}

To follow up the extensive characterization of splenic myeloid cells over the course of Py infection (Figure 12), a similar approach can be used to identify differences in cells that infiltrate infected tissues, such as the spleen and lungs, following secondary bacterial infection. By using similar methods (same cell isolation method, antibody staining and gating strategy), an experiment could determine whether or not there are differences in myeloid cells that enter the spleen (for Lm, Sp, even NTS) or lung (Sp only) at 3, 6, 12, 24 and 48 hours after the bacterial challenge between naïve and Pyinfected mice. A reduction in neutrophil migration to the liver 4 days after NTS infection has already been described in Py-infected mice compared to mice challenges with NTS alone [59]. Expanding on the characterization of cell types that have impaired or differential mobilization to sites of infection at various times after bacterial challenge 
would give additional insight into how changes in myeloid cell functions during malaria can impact innate immunity to bacterial coinfections. Since the spleen is impacted heavily during Plasmodium infection, and is vital to controlling bacterial infections during sepsis, a characterization of myeloid cells mobilization to the spleen following bacterial challenge would improve our understanding of exactly which cells are interacting with an invasive bacterial pathogen [47]. Dynamics of myeloid cell infiltration into lung tissue would also be of interest, given the potential for Plasmodium infections to induce lung pathology and the importance of neutrophils and monocytes during upper respiratory Pneumococcal infection [81, 172, 200]. Additional experiments using a fluorescent bacteria could determine which myeloid cell subsets are able to uptake bacteria and a time-course study, where investigators could look at the presence and abundance of bacteria within a variation cell types over the course of the bacterial infection in naïve and Py-infected mice to understand which cell types are involved with the uptake of bacteria, as well as the ability of those cells to control the infection. Further in vitro and ex vivo functional experiments can then be performed on the cells to explore potential Plasmodium-driven mechanisms of impaired anti-bacterial functions.

A different approach that would enhance the sensitivity, increase number of surface proteins that can be analyzed, and eliminate the complications of autofluorescence is with the cytometry by time-of-flight or CyTOF method [201, 202]. By utilizing this more advanced method, splenic myeloid cell populations could be identified with higher clarity and it would be possible to identify more distinct trends than I was able to obtain with the method described in this work. 


\section{Identifying and characterizing the contribution of a defective serum component in elevated susceptibility to bacterial coinfections in malaria}

A serum factor that was identified in Figure 15, where we saw decreased ROS production from PMNs infected with heat-treated Sp that had been opsonized with serum collected from Py-infected mice (Py-ops Ht Sp). This effect occurred in PMNs from

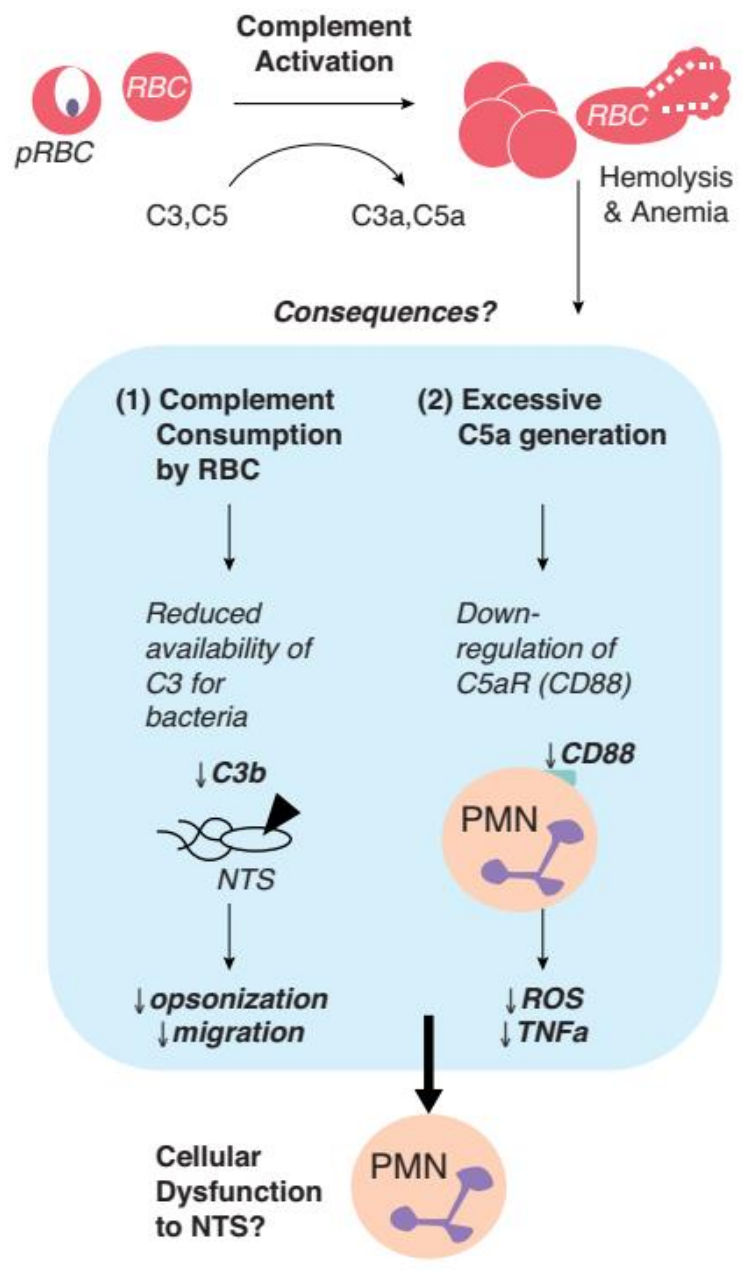
spleens of both naïve and Py-infected mice, but Py-ops Ht Sp exacerbated the already significant ROS production defect compared to cells from naïve mice. Interestingly, cells from Py-infected mice infected with naïve serumopsonized Sp and naïve mouse cells infected with Py-ops Ht Sp produced comparable levels of ROS. These data suggest that, in addition to an underlying defect in ROS production, a defective serum component in Pyinfected mice contributes to the Py-

Figure 26. Complement depletion during malaria. Adapted from Mooney et al. 2018 Journal of Leukocyte Biology. "Malaria, anemia, and bacterial disease: A neutrophil problem?" Malaria is accompanied by systemic complement activation and deposition on infected and uninfected RBCs. This decreases concentrations of circulating C3 and C5, and generates anaphylatoxins (C3a and C5a), potentially impacting important antibacterial PMN functions and contributing to increased risk of bacteremia during malaria. 
dependent impaired ROS response. There is strong rationale to conduct further research on the role of serum-opsonizing factors, namely complement factors, given that it is well established that Plasmodium infection activates and eventually functionally depletes complement components $[120,203,204]$. The connection between complement deficiency was discussed recently[60], where the authors postulate that Plasmodiumdriven complement depletion relates to decreased opsonization of NTS, lower neutrophil migration, ROS and TNF $\alpha$ production, and contribute to impairments in other PMN antimicrobial functions in response to NTS (Figure 26). The first steps needed to continue investigating the role of complement in Plasmodium-driven susceptibility would to verify that the serum factor involved in the decreased ROS production (Fig. 15) is in fact a complement component. A simple way to test this would be to heat-inactivate serum from both naïve and Py-infected mice and repeat the DCF ROS experiments with heat-treated Sp. If a decrease in ROS is found in cells infected with heat-inactivated naïve mouse serum opsonized Ht-Sp, the contribution of opsonizing antibodies can be ruled out, indicating the defective serum factor in Py-infected mice is a heat-labile complement factor. Conversely, experiments could be performed where individual complement components could be added to serum from Py-infected mice and incubated with Sp preparations prior to infection in order to identify precisely which complement protein is responsible for the Py-induced serum defect observed in this work. After a complement factor is identified in vitro, it can be verified in an in vivo co-infection experiment using a knockout mouse strain that lacks the gene coding for the protein of interest. 
Quantitative analysis of the complement components in serum of naïve vs. Pyinfected mice would serve as a preliminary screen in an effort to identify a specific protein that could be responsible for the defective ROS production function. This could be done either with comparing protein quantities in serum between naïve and Py-infected mouse serum via Western Blot, or via detection with a multiplex ELISA kit for complement factors or a flow cytometric analysis. Another approach could be performed in vivo with $\mathrm{Py}+\mathrm{Sp}$ or $\mathrm{Py}+\mathrm{Lm}$ coinfected mice compared with mice given bacteria alone with broad depletion of complement factors via treatment with Cobra Venom Factor (CVF) or depletion of specific complement components with some other method [127, 205]. An important interaction to consider in investigating interactions between Pneumococcus and complement is the Sp capsule, which in some cases binds directly to complement receptors or complement factors, and Sp capsule-mediated inhibition of complement activity $[126,127,206]$. For these reasons, it would be diligent to include capsulated, uncapsulated, and potentially a variety of capsular serotypes in any experiments involving Sp-complement interactions. Finally, it would also be interesting to investigate the contribution of the defective serum factor we found with defective PMN ROS production during Ht-ops Sp infection in an assay where Sp intracellular and extracellular viability is determined in coculture with PMNs isolated from naïve or Pyinfected mice, with the different manipulations of serum opsonins described above. These experiments would provide mechanistic insight into the Plasmodium-dependent susceptibility to invasive Pneumococcal disease that is currently lacking from the field that could lead to novel therapies for coinfected individuals. 


\section{Next steps in examining role of hemozoin}

The next steps in characterizing the role of hemozoin in suppression to bacterial coinfections will involve determining what specific innate immune cell populations have impaired antimicrobial functions and which important mechanism are impacted. This will require either visualization of hemozoin pigment within cells or an analysis method similar to what has been described with flow cytometry, taking advantage of the birefringent or ultraparamagnetic properties, as done in Frita et. al [207]. This will allow analysis of the percentage of cells with intracellular $\mathrm{Hz}$, and with the inclusion of antibodies specific to certain innate immune cell subsets, information about the identities of the cells and to some degree, the amount of $\mathrm{Hz}$ they uptake. In future experiments seeking to identify mechanisms of Hz-dependent impaired antimicrobial function, determining cell types with particular defects will be very helpful and obtaining this information from in vitro assays will pave the way for experiments with in vivo models where susceptibility to bacterial infection is analyzed in $\mathrm{Hz}$ treated mice and compared to untreated controls. Imagestream ${ }^{\circledR}$ equipment made by Amnis ${ }^{\circledR}$ (Seattle, WA), for example, would allow for analysis of fluorescence marker quantification that is paired to bright field images at the single cell level and analysis of intracellular $\mathrm{Hz}$ has been done with this equipment [184]. ROS detection and bacterial uptake, survival, and growth would also be detectible with use of DCF, as done in this dissertation, and a fluorescence expressing bacteria. Also, a fluorescent strain of Sp could be used in a DCF assay to potentially determine whether $\mathrm{Sp}$ is the source of the large amount ROS we observed in Figures 18 and 19. 
In addition to these experiments, more work is also needed to identify precisely which Hz-bound bioactive molecules are responsible for the immunomodulatory activity of $\mathrm{Hz}$ that we have observed. This can be achieved via careful molecular characterization of the different preparations of hemozoin described here. There is reason to suspect that the immunomodulatory effects we have observed in both the delayed clearance of Lm from spleens of PyHz-treated mice and the inability of PyHz-treated splenic phagocytes to control intracellular growth of Lm in vitro (Figures 22 and 21, respectively), is due to hydroxy fatty acids described by Schwarzer et al. [186]. Performing the experiments described in this work which involve $\mathrm{Hz}$, as well as those mentioned in future directions, with de-lipidation treatment used by Schwarzer and colleagues [186]on $\mathrm{Hz}$ will provide meaningful insight to move the project forward into high-impact mechanistic studies.

\section{RNA-seq analysis to characterize overall effect of hemozoin uptake on myeloid cell with systematic function analysis}

Currently, very little is known about how phagocyte antimicrobial functions change following the uptake of $\mathrm{Hz}$. One study has investigated changes in gene expression in human peripheral blood monocytes treated with $\mathrm{Hz}$ or $\mathrm{iRBCs}$ and found that $\mathrm{Hz}$ and $\mathrm{iRBC}$-treated cells were hyperresponsive in response to a second stimulus 3 days after $\mathrm{Hz}$ or iRBC exposure [112]. A similar RNA-seq approach could be used to examine gene expression of ultra-purified Hz-treated and untreated PMNs and macrophage cultures with multiple stimuli to determine specific mechanisms by which their effector functions are impaired. Such an analysis would compare mRNA levels of these cells with or without $\mathrm{Hz}$ treatment followed by stimulation with chemicals known 
to induce antimicrobial responses, including PMA, LPS, heat-treated Sp or

Staphylococcus aureus. Assessing gene expression in cells with or without $\mathrm{Hz}$ treatment following stimulation could uncover a pathway that is made defective by internalization or interaction with $\mathrm{Hz}$ in vitro and subsequent testing with gene silencing, knowckout mice, or inhibitory molecules specific to pathways of interest can be used to validate findings from RNA-seq analysis. The same method could also be used in comparing cells from Py-infected and naïve mice or $P$. falciparum infected individuals compared to healthy controls. Altogether, the combination of gene expression analysis cells with or without $\mathrm{Hz}$ treatment and cells from patients with or without malaria would provide insight to which defects in phagocyte antimicrobial function are specific to uptake or interaction with $\mathrm{Hz}$, and which are independent of $\mathrm{Hz}$ and caused by some other factor during Plasmodium infection.

\section{Contribution to the Field}

Our findings provide evidence that there are multiple factors involved in Plasmodium-induced suppression of antibacterial innate immunity. Combinations of these factors likely contribute to the enhanced susceptibility to the different bacterial pathogens seen in conjunction with malaria in sub-Saharan Africa. Given the increased mortality rate associated with malaria and invasive bacterial coinfections, these results may provide insight into new approaches to treat those individuals. Finally, this report provides the first description, to our knowledge, of a murine model system of Plasmodium and S. pneumoniae coinfection. In this model, Plasmodium infections impair host immunity to S. pneumoniae, which resulted in increased mortality. As $S$.

pneumoniae is one of the most common bacteria identified in individuals with invasive 
bacterial infections during malaria, this model has the potential to provide further

knowledge into the dynamics of this coinfection and identify approaches to treat this

coinfection. A summary of the mechanisms of Plasmodium-induced immunosuppression

that have been experimentally explored in models of NTS, Lm, and Sp coinfections can

be found in Figure 27.

\section{Potential Mechanisms of Plasmodium-immune}

\section{suppression}

- Hemolysis resulting from Plasmodium infection inducing heme oxygenase-1 limiting oxidative burst

- IL-10 blunting of granulocyte chemotaxis and infiltration to infected tissues

- Impaired ROS generation

- Serum component

- Defective control of intracellular bacterial survival Hz+bound bioactive molecules

- $\mathrm{Hz}$ - impaired control of intracellular bacterial survival

- $\mathrm{Hz}$ - weakens anti-bacterial immunity in vivo

- $\mathrm{Hz}$ - decreases ROS production of neutrophils

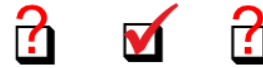

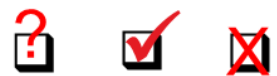

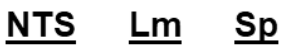

$\nabla$ X

$\nabla \quad \nabla \quad \nabla$

\$ $\nabla$

$\nabla \quad \mathbb{\nabla}$

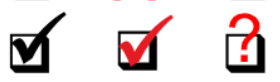

- $\mathbf{X}$ \

Black: Previously known

Figure 27. Potential mechanisms of Plasmodium-driven immune suppression to bacterial

coinfections. List of mechanisms of Plasmodium-induced impaired immunity to bacterial co-infections for non-Typhoid Salmonella (NTS), Listeria monocytogenes $(\mathrm{Lm})$, and Streptococcus pneumoniae (Sp). Check mark = mechanism is known be involved, " $X$ " mark = no experimental evidence that suggests mechanism is involved, question mark = mechanism has not been tested for indicated bacterial pathogen. Black marks indicate previously known mechanisms while the red marks indicate the results from this dissertation. See text for references and details. 


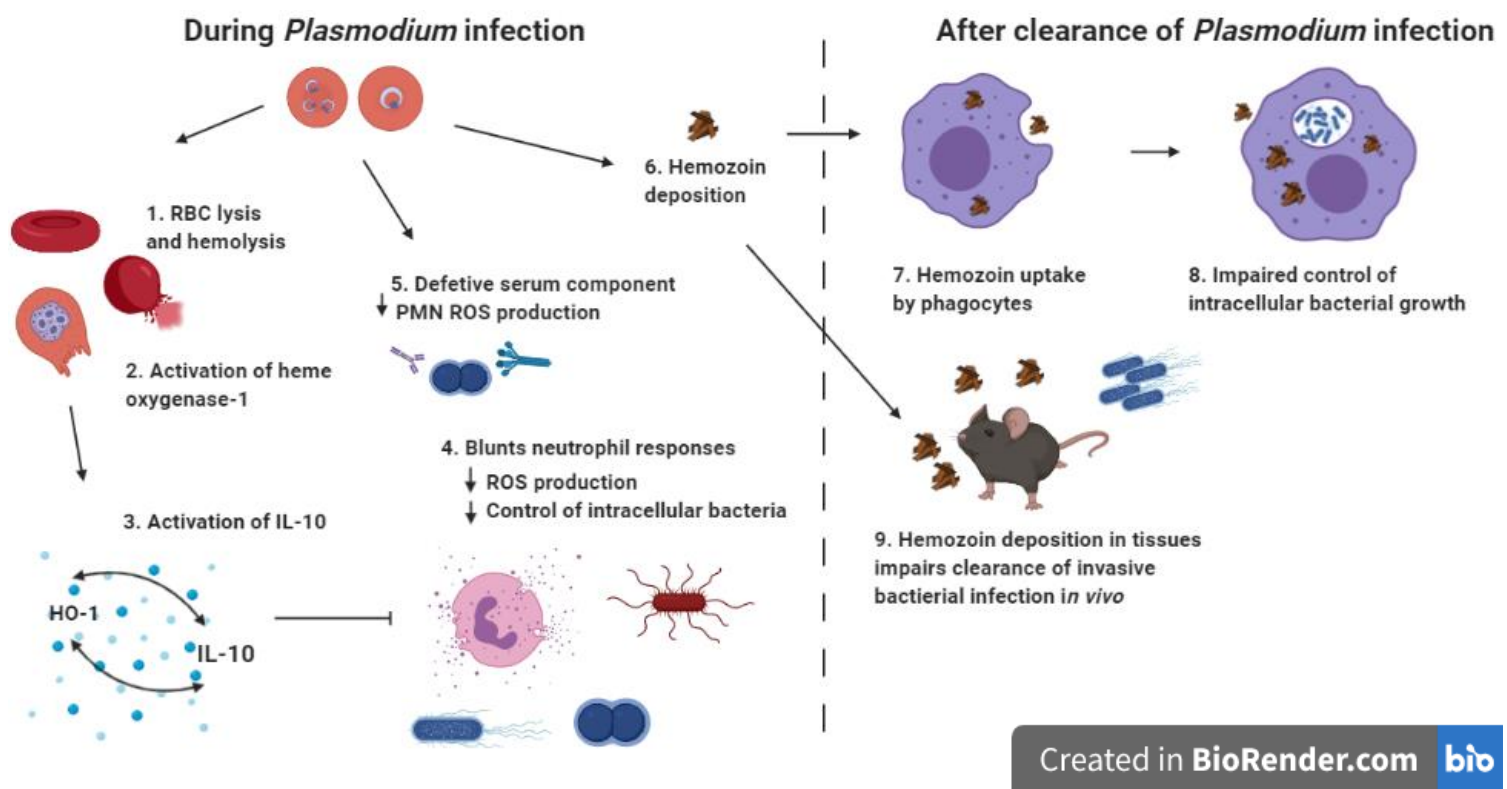

Figure 28. Mechanisms of Plasmodium infection-driven suppression of antibacterial innate immunity to invasive coinfections. 1) Rupture of parasitized red blood cells during the blood stage of malaria results in hemolysis and the activation of HO-1(2) and IL-10 (3), which initiates a positive feedback loop of activation. Both HO-1 and IL-10 are known to blunt neutrophil responses such as ROS production and impair innate immunity to NTS, while only IL-10 has been found to impair immunity to Sp. Also, via mechanisms that are not yet well understood, Plasmodium infection impairs the ability of splenic phagocytes to control the intracellular growth of bacteria (4). Activation of the complement system occurs during blood stage of Plasmodium infection, leading to their functional depletion. This defect in complement or parasite driven changes to opsonizing antibodies are likely responsible for our observations that a defective serum factor impairs the ROS response of neutrophils (5). Hemozoin crystals are byproducts of the parasitic digestion of hemoglobin and are released into circulation upon rupture of parasitized red blood cells. These crystals are deposited in tissues (6) where they are internalized by phagocytes (7) which impairs their ability to control growth of intracellular bacteria (8) and causes systemic defects in the ability of the innate immune response to clear an invasive bacterial infection (9). 


\section{REFERENCES}

1. Organization, W.H., World Malaria Report 2018. 2018, Geneva: World Health Organization.

2. Organization, W.H., World malaria report 2019. Geneva: Wolrd Health Organization, 2019.

3. Cox, F.E., History of the discovery of the malaria parasites and their vectors. Parasit Vectors, 2010. 3(1): p. 5.

4. Poinar, G., Jr. and S.R. Telford, Jr., Paleohaemoproteus burmacis gen. n., sp. n. (Haemospororida: Plasmodiidae) from an Early Cretaceous biting midge (Diptera: Ceratopogonidae). Parasitology, 2005. 131(Pt 1): p. 79-84.

5. $\quad$ Baird, J.K., Malaria zoonoses. Travel Med Infect Dis, 2009. 7(5): p. 269-77.

6. White, N.J., Plasmodium knowlesi: the fifth human malaria parasite. Clin Infect Dis, 2008. 46(2): p. 172-3.

7. Arese, P., How genetics and biology helped humanity to survive falciparum malaria. Parassitologia, 2006. 48(4): p. 553-9.

8. Perkins, S.L., Malaria's many mates: past, present, and future of the systematics of the order Haemosporida. J Parasitol, 2014. 100(1): p. 11-25.

9. Michalakis, Y. and F. Renaud, Malaria: Evolution in vector control. Nature, 2009. 462(7271): p. 298-300.

10. Tavares, J., et al., Role of host cell traversal by the malaria sporozoite during liver infection. J Exp Med, 2013. 210(5): p. 905-15.

11. Cowman, A.F., et al., Malaria: Biology and Disease. Cell, 2016. 167(3): p. 610624.

12. Mo, A.X. and G. McGugan, Understanding the Liver-Stage Biology of Malaria Parasites: Insights to Enable and Accelerate the Development of a Highly Efficacious Vaccine. Am J Trop Med Hyg, 2018. 99(4): p. 827-832.

13. Sturm, A., et al., Manipulation of host hepatocytes by the malaria parasite for delivery into liver sinusoids. Science, 2006. 313(5791): p. 1287-90.

14. Mideo, N., et al., The Cinderella syndrome: why do malaria-infected cells burst at midnight? Trends Parasitol, 2013. 29(1): p. 10-6.

15. Hawking, F., M.J. Worms, and K. Gammage, 24-and 48-hour cycles of malaria parasites in the blood; their purpose, production and control. Trans R Soc Trop Med Hyg, 1968. 62(6): p. 731-65.

16. Garnham, P.C. and K.G. Powers, Periodicity of infectivity of plasmodial gametocytes: the "Hawking phenomenon". Int J Parasitol, 1974. 4(1): p. 103-6.

17. Silver, A.C., et al., The circadian clock controls toll-like receptor 9-mediated innate and adaptive immunity. Immunity, 2012. 36(2): p. 251-61.

18. Keller, M., et al., A circadian clock in macrophages controls inflammatory immune responses. Proc Natl Acad Sci U S A, 2009. 106(50): p. 21407-12. 
19. Dondorp, A.M., et al., The relationship between age and the manifestations of and mortality associated with severe malaria. Clin Infect Dis, 2008. 47(2): p. 151-7.

20. Reyburn, H., et al., Association of transmission intensity and age with clinical manifestations and case fatality of severe Plasmodium falciparum malaria. JAMA, 2005. 293(12): p. 1461-70.

21. Bartoloni, A. and L. Zammarchi, Clinical aspects of uncomplicated and severe malaria. Mediterr J Hematol Infect Dis, 2012. 4(1): p. e2012026.

22. Krajden, S., et al., Prolonged infection with Plasmodium falciparum in a semiimmune patient. Trans R Soc Trop Med Hyg, 1991. 85(6): p. 731-2.

23. Greenwood, T., et al., Febrile Plasmodium falciparum malaria 4 years after exposure in a man with sickle cell disease. Clin Infect Dis, 2008. 47(4): p. e39-41.

24. Szmitko, P.E., M.L. Kohn, and A.E. Simor, Plasmodium falciparum malaria occurring 8 years after leaving an endemic area. Diagn Microbiol Infect Dis, 2009. 63(1): p. 105-7.

25. Silal, S.P., et al., Towards malaria elimination in Mpumalanga, South Africa: a population-level mathematical modelling approach. Malar J, 2014. 13: p. 297.

26. Larsen, D.A., et al., Population-wide malaria testing and treatment with rapid diagnostic tests and artemether-lumefantrine in southern Zambia: a community randomized step-wedge control trial design. Am J Trop Med Hyg, 2015. 92(5): p. 913-921.

27. Cook, J., et al., Mass screening and treatment on the basis of results of a Plasmodium falciparum-specific rapid diagnostic test did not reduce malaria incidence in Zanzibar. J Infect Dis, 2015. 211(9): p. 1476-83.

28. Mishra, N., et al., Surveillance of artemisinin resistance in Plasmodium falciparum in India using the kelch13 molecular marker. Antimicrob Agents Chemother, 2015. 59(5): p. 2548-53.

29. Dhiman, S., Are malaria elimination efforts on right track? An analysis of gains achieved and challenges ahead. Infect Dis Poverty, 2019. 8(1): p. 14.

30. World malaria situation in 1994. Part II. Wkly Epidemiol Rec, 1997. 72(37): p. 277-83.

31. Gallup, J.L. and J.D. Sachs, The economic burden of malaria. Am J Trop Med Hyg, 2001. 64(1-2 Suppl): p. 85-96.

32. Sachs, J. and P. Malaney, The economic and social burden of malaria. Nature, 2002. 415(6872): p. 680-5.

33. Hertig, E., Distribution of Anopheles vectors and potential malaria transmission stability in Europe and the Mediterranean area under future climate change. Parasit Vectors, 2019. 12(1): p. 18.

34. Mota, M.M., J.C. Hafalla, and A. Rodriguez, Migration through host cells activates Plasmodium sporozoites for infection. Nat Med, 2002. 8(11): p. 131822.

35. Gowda, D.C. and X. Wu, Parasite Recognition and Signaling Mechanisms in Innate Immune Responses to Malaria. Front Immunol, 2018. 9: p. 3006.

36. Aitken, E.H., A. Alemu, and S.J. Rogerson, Neutrophils and Malaria. Front Immunol, 2018. 9: p. 3005.

37. Brinkmann, V., et al., Role of macrophages in malaria: O2 metabolite production and phagocytosis by splenic macrophages during lethal Plasmodium berghei and 
self-limiting Plasmodium yoelii infection in mice. Infect Immun, 1984. 44(3): p. 743-6.

38. Buffet, P.A., et al., The pathogenesis of Plasmodium falciparum malaria in humans: insights from splenic physiology. Blood, 2011. 117(2): p. 381-92.

39. Borges da Silva, H., et al., Splenic Macrophage Subsets and Their Function during Blood-Borne Infections. Front Immunol, 2015. 6: p. 480.

40. Leitner, W.W., et al., Role of Opsonophagocytosis in Immune Protection against Malaria. Vaccines (Basel), 2020. 8(2).

41. Long, C.A. and F. Zavala, Immune Responses in Malaria. Cold Spring Harb Perspect Med, 2017. 7(8).

42. Scott, J.A., et al., Relation between falciparum malaria and bacteraemia in Kenyan children: a population-based, case-control study and a longitudinal study. Lancet, 2011. 378(9799): p. 1316-23.

43. Auma, M.A., et al., Malaria is an uncommon cause of adult sepsis in southwestern Uganda. Malar J, 2013. 12: p. 146.

44. Morton, B., et al., The Early Recognition and Management of Sepsis in SubSaharan African Adults: A Systematic Review and Meta-Analysis. Int J Environ Res Public Health, 2018. 15(9).

45. Reddy, E.A., A.V. Shaw, and J.A. Crump, Community-acquired bloodstream infections in Africa: a systematic review and meta-analysis. Lancet Infect Dis, 2010. 10(6): p. 417-32.

46. Were, T., et al., Bacteremia in Kenyan children presenting with malaria. J Clin Microbiol, 2011. 49(2): p. 671-6.

47. Gomez-Perez, G.P., et al., Plasmodium falciparum malaria and invasive bacterial co-infection in young African children: the dysfunctional spleen hypothesis. Malar J, 2014. 13: p. 335.

48. Colombatti, R., et al., Malaria prevention reduces in-hospital mortality among severely ill tuberculosis patients: a three-step intervention in Bissau, GuineaBissau. BMC Infect Dis, 2011. 11: p. 57.

49. Berkley, J.A., et al., Bacteremia among children admitted to a rural hospital in Kenya. N Engl J Med, 2005. 352(1): p. 39-47.

50. Lalremruata, A., et al., Molecular identification of falciparum malaria and human tuberculosis co-infections in mummies from the Fayum depression (Lower Egypt). PLoS One, 2013. 8(4): p. e60307.

51. Walsh, A.L., et al., Bacteremia in febrile Malawian children: clinical and microbiologic features. Pediatr Infect Dis J, 2000. 19(4): p. 312-8.

52. Nielsen, M.V., et al., Clinical indicators for bacterial co-infection in Ghanaian children with P. falciparum infection. PLoS One, 2015. 10(4): p. e0122139.

53. Church, J. and K. Maitland, Invasive bacterial co-infection in African children with Plasmodium falciparum malaria: a systematic review. BMC Med, 2014. 12 : p. 31 .

54. Mourembou, G., et al., Co-circulation of Plasmodium and Bacterial DNAs in Blood of Febrile and Afebrile Children from Urban and Rural Areas in Gabon. Am J Trop Med Hyg, 2016. 95(1): p. 123-32.

55. Decuypere, S., et al., Diagnosis of Bacterial Bloodstream Infections: A $16 \mathrm{~S}$ Metagenomics Approach. PLoS Negl Trop Dis, 2016. 10(2): p. e0004470. 
56. Mabey, D.C., A. Brown, and B.M. Greenwood, Plasmodium falciparum malaria and Salmonella infections in Gambian children. J Infect Dis, 1987. 155(6): p. 1319-21.

57. Crum-Cianflone, N.F., Salmonellosis and the gastrointestinal tract: more than just peanut butter. Curr Gastroenterol Rep, 2008. 10(4): p. 424-31.

58. MacLennan, C.A. and M.M. Levine, Invasive nontyphoidal Salmonella disease in Africa: current status. Expert Rev Anti Infect Ther, 2013. 11(5): p. 443-6.

59. Lokken, K.L., et al., Malaria parasite infection compromises control of concurrent systemic non-typhoidal Salmonella infection via IL-10-mediated alteration of myeloid cell function. PLoS Pathog, 2014. 10(5): p. e1004049.

60. Mooney, J.P., L.J. Galloway, and E.M. Riley, Malaria, anemia, and invasive bacterial disease: A neutrophil problem? J Leukoc Biol, 2019. 105(4): p. 645655.

61. Cunnington, A.J., et al., Prolonged neutrophil dysfunction after Plasmodium falciparum malaria is related to hemolysis and heme oxygenase-1 induction. J Immunol, 2012. 189(11): p. 5336-46.

62. Cunnington, A.J., et al., Malaria impairs resistance to Salmonella through hemeand heme oxygenase-dependent dysfunctional granulocyte mobilization. Nat Med, 2011. 18(1): p. 120-7.

63. Nyirenda, T.S., et al., Immunological bases of increased susceptibility to invasive nontyphoidal Salmonella infection in children with malaria and anaemia. Microbes Infect, 2018. 20(9-10): p. 589-598.

64. Nyirenda, T.S., et al., Loss of Humoral and Cellular Immunity to Invasive Nontyphoidal Salmonella during Current or Convalescent Plasmodium falciparum Infection in Malawian Children. Clin Vaccine Immunol, 2017. 24(7).

65. Valadas, E., et al., Tuberculosis with malaria or HIV co-infection in a large hospital in Luanda, Angola. J Infect Dev Ctries, 2013. 7(3): p. 269-72.

66. Range, N., et al., HIV and parasitic co-infections in tuberculosis patients: a crosssectional study in Mwanza, Tanzania. Ann Trop Med Parasitol, 2007. 101(4): p. 343-51.

67. Frita, R.M.R.C., Malaria and Tuberculosis co-infection: role for Hemozoin immunosyppression?, in Unidade de Microbiologia Molecular e Infecção do Instituto de Medicina Molecular. 2014, Universidade de Lisboa, Faculdade de Medicina: Lisbon, Portugal. p. 165.

68. Pisell, T.L., et al., Immune activation and induction of HIV-1 replication within CD14 macrophages during acute Plasmodium falciparum malaria coinfection. AIDS, 2002. 16(11): p. 1503-9.

69. Kublin, J.G., et al., Effect of Plasmodium falciparum malaria on concentration of HIV-1-RNA in the blood of adults in rural Malawi: a prospective cohort study. Lancet, 2005. 365(9455): p. 233-40.

70. Renia, L. and S.M. Potter, Co-infection of malaria with HIV: an immunological perspective. Parasite Immunol, 2006. 28(11): p. 589-95.

71. Cuadros, D.F., A.J. Branscum, and P.H. Crowley, HIV-malaria co-infection: effects of malaria on the prevalence of HIV in East sub-Saharan Africa. Int J Epidemiol, 2011. 40(4): p. 931-9. 
72. Andreani, G., et al., An in vitro co-infection model to study Plasmodium falciparum-HIV-1 interactions in human primary monocyte-derived immune cells. J Vis Exp, 2012(66): p. e4166.

73. Diou, J., et al., Malaria hemozoin modulates susceptibility of immature monocytederived dendritic cells to HIV-1 infection by inducing a mature-like phenotype. Cell Microbiol, 2010. 12(5): p. 615-25.

74. Diou, J., et al., Dendritic cells derived from hemozoin-loaded monocytes display a partial maturation phenotype that promotes HIV-1 trans-infection of CD4+ $T$ cells and virus replication. J Immunol, 2010. 184(6): p. 2899-907.

75. Nadjm, B., et al., WHO guidelines for antimicrobial treatment in children admitted to hospital in an area of intense Plasmodium falciparum transmission: prospective study. BMJ, 2010. 340: p. c1350.

76. Njim, T., et al., Identifying risk factors for the development of sepsis during adult severe malaria. Malar J, 2018. 17(1): p. 278.

77. Dong, E., H. Du, and L. Gardner, An interactive web-based dashboard to track COVID-19 in real time. Lancet Infect Dis, 2020. 20(5): p. 533-534.

78. Laroque, A., et al., Genetic control of susceptibility to infection with Plasmodium chabaudi chabaudi AS in inbred mouse strains. Genes Immun, 2012. 13(2): p. 155-63.

79. Stevenson, M.M., J.J. Lyanga, and E. Skamene, Murine malaria: genetic control of resistance to Plasmodium chabaudi. Infect Immun, 1982. 38(1): p. 80-8.

80. Fortin, A., et al., Genetic control of blood parasitaemia in mouse malaria maps to chromosome 8. Nat Genet, 1997. 17(4): p. 382-3.

81. Deroost, K., et al., Hemozoin induces lung inflammation and correlates with malaria-associated acute respiratory distress syndrome. Am J Respir Cell Mol Biol, 2013. 48(5): p. 589-600.

82. Coban, C., et al., Purified malaria pigment (hemozoin) enhances dendritic cell maturation and modulates the isotype of antibodies induced by a DNA vaccine. Infect Immun, 2002. 70(7): p. 3939-43.

83. Deroost, K., et al., Improved methods for haemozoin quantification in tissues yield organ-and parasite-specific information in malaria-infected mice. Malar J, 2012. 11: p. 166.

84. Barrera, V., et al., Host fibrinogen stably bound to hemozoin rapidly activates monocytes via TLR-4 and CD11b/CD18-integrin: a new paradigm of hemozoin action. Blood, 2011. 117(21): p. 5674-82.

85. Schwarzer, E., F. Turrini, and P. Arese, A luminescence method for the quantitative determination of phagocytosis of erythrocytes, of malaria-parasitized erythrocytes and of malarial pigment. Br J Haematol, 1994. 88(4): p. 740-5.

86. Lee, T.S. and L.Y. Chau, Heme oxygenase-1 mediates the anti-inflammatory effect of interleukin-10 in mice. Nat Med, 2002. 8(3): p. 240-6.

87. Usuf, E., et al., Pneumococcal carriage in sub-Saharan Africa--a systematic review. PLoS One, 2014. 9(1): p. e85001.

88. Henriques-Normark, B. and E.I. Tuomanen, The pneumococcus: epidemiology, microbiology, and pathogenesis. Cold Spring Harb Perspect Med, 2013. 3(7).

89. Song, J.Y., B.W. Eun, and M.H. Nahm, Diagnosis of pneumococcal pneumonia: current pitfalls and the way forward. Infect Chemother, 2013. 45(4): p. 351-66. 
90. Nadjm, B., et al., Severe febrile illness in adult hospital admissions in Tanzania: a prospective study in an area of high malaria transmission. Trans $\mathrm{R}$ Soc Trop Med Hyg, 2012. 106(11): p. 688-95.

91. Bassat, Q., et al., Severe malaria and concomitant bacteraemia in children admitted to a rural Mozambican hospital. Trop Med Int Health, 2009. 14(9): p. 1011-9.

92. Amani, V., et al., Involvement of IFN-gamma receptor-medicated signaling in pathology and anti-malarial immunity induced by Plasmodium berghei infection. Eur J Immunol, 2000. 30(6): p. 1646-55.

93. Day, N.P., et al., The prognostic and pathophysiologic role of pro- and antiinflammatory cytokines in severe malaria. J Infect Dis, 1999. 180(4): p. 128897.

94. Grau, G.E., et al., Tumor necrosis factor and disease severity in children with falciparum malaria. N Engl J Med, 1989. 320(24): p. 1586-91.

95. Anstey, N.M., et al., Nitric oxide in Tanzanian children with malaria: inverse relationship between malaria severity and nitric oxide production/nitric oxide synthase type 2 expression. J Exp Med, 1996. 184(2): p. 557-67.

96. Omer, F.M., J.A. Kurtzhals, and E.M. Riley, Maintaining the immunological balance in parasitic infections: a role for TGF-beta? Parasitol Today, 2000. 16(1): p. 18-23.

97. Omer, F.M. and E.M. Riley, Transforming growth factor beta production is inversely correlated with severity of murine malaria infection. J Exp Med, 1998. 188(1): p. 39-48.

98. Othoro, C., et al., A low interleukin-10 tumor necrosis factor-alpha ratio is associated with malaria anemia in children residing in a holoendemic malaria region in western Kenya. J Infect Dis, 1999. 179(1): p. 279-82.

99. Mooney, J.P., et al., The mucosal inflammatory response to non-typhoidal Salmonella in the intestine is blunted by IL-10 during concurrent malaria parasite infection. Mucosal Immunol, 2014. 7(6): p. 1302-11.

100. Tilney, L.G. and D.A. Portnoy, Actin filaments and the growth, movement, and spread of the intracellular bacterial parasite, Listeria monocytogenes. J Cell Biol, 1989. 109(4 Pt 1): p. 1597-608.

101. Fritsche, G., et al., Regulatory interactions between iron and nitric oxide metabolism for immune defense against Plasmodium falciparum infection. J Infect Dis, 2001. 183(9): p. 1388-94.

102. Kern, P., et al., Elevated tumor necrosis factor alpha and interleukin-6 serum levels as markers for complicated Plasmodium falciparum malaria. Am J Med, 1989. 87(2): p. 139-43.

103. McCall, M.B. and R.W. Sauerwein, Interferon-gamma--central mediator of protective immune responses against the pre-erythrocytic and blood stage of malaria. J Leukoc Biol, 2010. 88(6): p. 1131-43.

104. Villegas-Mendez, A., et al., IFN-gamma-producing CD4+ T cells promote experimental cerebral malaria by modulating $C D 8+T$ cell accumulation within the brain. J Immunol, 2012. 189(2): p. 968-79.

105. Huang, S., et al., Immune response in mice that lack the interferon-gamma receptor. Science, 1993. 259(5102): p. 1742-5. 
106. Kocks, C., et al., L. monocytogenes-induced actin assembly requires the actA gene product, a surface protein. Cell, 1992. 68(3): p. 521-31.

107. Pitts, M.G. and S.E.F. D'Orazio, A Comparison of Oral and Intravenous Mouse Models of Listeriosis. Pathogens, 2018. 7(1).

108. Lokken, K.L., et al., Malaria Parasite-Mediated Alteration of Macrophage Function and Increased Iron Availability Predispose to Disseminated Nontyphoidal Salmonella Infection. Infect Immun, 2018. 86(9).

109. Mooney, J.P., et al., Haemolysis and haem oxygenase-1 induction during persistent "asymptomatic" malaria infection in Burkinabe children. Malar J, 2018. 17(1): p. 253.

110. Frita, R., et al., In Vivo Hemozoin Kinetics after Clearance of Plasmodium berghei Infection in Mice. Malar Res Treat, 2012. 2012: p. 373086.

111. Netea, M.G., Training innate immunity: the changing concept of immunological memory in innate host defence. Eur J Clin Invest, 2013. 43(8): p. 881-4.

112. Schrum, J.E., et al., Cutting Edge: Plasmodium falciparum Induces Trained Innate Immunity. J Immunol, 2018. 200(4): p. 1243-1248.

113. Franklin, B.S., et al., Malaria primes the innate immune response due to interferon-gamma induced enhancement of toll-like receptor expression and function. Proc Natl Acad Sci U S A, 2009. 106(14): p. 5789-94.

114. Ataide, M.A., et al., Malaria-induced NLRP12/NLRP3-dependent caspase-1 activation mediates inflammation and hypersensitivity to bacterial superinfection. PLoS Pathog, 2014. 10(1): p. e1003885.

115. Lujan, M., et al., Influence of pneumococcal serotype group on outcome in adults with bacteraemic pneumonia. Eur Respir J, 2010. 36(5): p. 1073-9.

116. van der Sluijs, K.F., et al., IL-10 is an important mediator of the enhanced susceptibility to pneumococcal pneumonia after influenza infection. J Immunol, 2004. 172(12): p. 7603-9.

117. Leoratti, F.M., et al., Neutrophil paralysis in Plasmodium vivax malaria. PLoS Negl Trop Dis, 2012. 6(6): p. e1710.

118. Peyron, F., et al., High levels of circulating IL-10 in human malaria. Clin Exp Immunol, 1994. 95(2): p. 300-3.

119. White, C.E., et al., Plasmodium suppresses expansion of $T$ cell responses to heterologous infections. J Immunol, 2015. 194(2): p. 697-708.

120. Nyakoe, N.K., et al., Complement consumption in children with Plasmodium falciparum malaria. Malar J, 2009. 8: p. 7.

121. Dey, S., et al., Association of heme oxygenase 1 with the restoration of liver function after damage in murine malaria by Plasmodium yoelii. Infect Immun, 2014. 82(8): p. 3113-26.

122. Mebius, R.E. and G. Kraal, Structure and function of the spleen. Nat Rev Immunol, 2005. 5(8): p. 606-16.

123. den Haan, J.M. and G. Kraal, Innate immune functions of macrophage subpopulations in the spleen. J Innate Immun, 2012. 4(5-6): p. 437-45.

124. Perez, O.A., et al., CD169(+) macrophages orchestrate innate immune responses by regulating bacterial localization in the spleen. Sci Immunol, 2017. 2(16).

125. Lanoue, A., et al., SIGN-RI contributes to protection against lethal pneumococcal infection in mice. J Exp Med, 2004. 200(11): p. 1383-93. 
126. Kang, Y.S., et al., The C-type lectin SIGN-RI mediates uptake of the capsular polysaccharide of Streptococcus pneumoniae in the marginal zone of mouse spleen. Proc Natl Acad Sci U S A, 2004. 101(1): p. 215-20.

127. Kang, Y.S., et al., A dominant complement fixation pathway for pneumococcal polysaccharides initiated by SIGN-R1 interacting with C1q. Cell, 2006. 125(1): p. 47-58.

128. Gough, P.J. and S. Gordon, The role of scavenger receptors in the innate immune system. Microbes Infect, 2000. 2(3): p. 305-11.

129. Chen, Y., et al., A regulatory role for macrophage class A scavenger receptors in TLR4-mediated LPS responses. Eur J Immunol, 2010. 40(5): p. 1451-60.

130. Elomaa, O., et al., Cloning of a novel bacteria-binding receptor structurally related to scavenger receptors and expressed in a subset of macrophages. Cell, 1995. 80(4): p. 603-9.

131. Aichele, P., et al., Macrophages of the splenic marginal zone are essential for trapping of blood-borne particulate antigen but dispensable for induction of specific T cell responses. J Immunol, 2003. 171(3): p. 1148-55.

132. Backer, R., et al., Effective collaboration between marginal metallophilic macrophages and CD8+dendritic cells in the generation of cytotoxic T cells. Proc Natl Acad Sci U S A, 2010. 107(1): p. 216-21.

133. Kirby, A.C., et al., SIGNRl-negative red pulp macrophages protect against acute streptococcal sepsis after Leishmania donovani-induced loss of marginal zone macrophages. Am J Pathol, 2009. 175(3): p. 1107-15.

134. Beattie, L., et al., CD8+ T lymphocyte-mediated loss of marginal metallophilic macrophages following infection with Plasmodium chabaudi chabaudi AS. J Immunol, 2006. 177(4): p. 2518-26.

135. Bidegain, F., et al., Acute Plasmodium falciparum malaria following splenectomy for suspected lymphoma in 2 patients. Clin Infect Dis, 2005. 40(11): p. e97-100.

136. Leoni, S., et al., The hyper-reactive malarial splenomegaly: a systematic review of the literature. Malar J, 2015. 14: p. 185.

137. Urban, B.C., et al., Fatal Plasmodium falciparum malaria causes specific patterns of splenic architectural disorganization. Infect Immun, 2005. 73(4): p. 1986-94.

138. Stevenson, M.M. and G. Kraal, Histological changes in the spleen and liver of C57BL/6 and A/J mice during Plasmodium chabaudi AS infection. Exp Mol Pathol, 1989. 51(1): p. 80-95.

139. Nagaoka, K., et al., Association of SIGNRI with TLR4-MD-2 enhances signal transduction by recognition of LPS in gram-negative bacteria. Int Immunol, 2005. 17(7): p. 827-36.

140. Bronte, V. and M.J. Pittet, The spleen in local and systemic regulation of immunity. Immunity, 2013. 39(5): p. 806-18.

141. Delano, M.J., et al., Sepsis induces early alterations in innate immunity that impact mortality to secondary infection. J Immunol, 2011. 186(1): p. 195-202.

142. Deniset, J.F., et al., Splenic Ly6G(high) mature and Ly6G(int) immature neutrophils contribute to eradication of S. pneumoniae. J Exp Med, 2017. 214(5): p. 1333-1350.

143. Ozarslan, N., J.F. Robinson, and S.L. Gaw, Circulating Monocytes, Tissue Macrophages, and Malaria. J Trop Med, 2019. 2019: p. 3720838. 
144. Olliaro, P., et al., Hematologic parameters in pediatric uncomplicated Plasmodium falciparum malaria in sub-Saharan Africa. Am J Trop Med Hyg, 2011. 85(4): p. 619-25.

145. Teirlinck, A.C., et al., Plasmodium falciparum Infection of Human Volunteers Activates Monocytes and CD16+ Dendritic Cells and Induces Upregulation of CD16 and CD1c Expression. Infect Immun, 2015. 83(9): p. 3732-9.

146. Ioannidis, L.J., C.Q. Nie, and D.S. Hansen, The role of chemokines in severe malaria: more than meets the eye. Parasitology, 2014. 141(5): p. 602-13.

147. Chan, J.A., et al., Patterns of protective associations differ for antibodies to $P$. falciparum-infected erythrocytes and merozoites in immunity against malaria in children. Eur J Immunol, 2017. 47(12): p. 2124-2136.

148. Zhou, J., et al., CD14(hi)CD16+ monocytes phagocytose antibody-opsonised Plasmodium falciparum infected erythrocytes more efficiently than other monocyte subsets, and require CD16 and complement to do so. BMC Med, 2015. 13: p. 154.

149. McGilvray, I.D., et al., Nonopsonic monocyte/macrophage phagocytosis of Plasmodium falciparum-parasitized erythrocytes: a role for CD36 in malarial clearance. Blood, 2000. 96(9): p. 3231-40.

150. Sponaas, A.M., et al., Migrating monocytes recruited to the spleen play an important role in control of blood stage malaria. Blood, 2009. 114(27): p. 552231.

151. Mandala, W.L., et al., Monocyte activation and cytokine production in Malawian children presenting with P. falciparum malaria. Parasite Immunol, 2016. 38(5): p. 317-25.

152. Nunes, P., N. Demaurex, and M.C. Dinauer, Regulation of the NADPH oxidase and associated ion fluxes during phagocytosis. Traffic, 2013. 14(11): p. 1118-31.

153. Brown, J.S., et al., The classical pathway is the dominant complement pathway required for innate immunity to Streptococcus pneumoniae infection in mice. Proc Natl Acad Sci U S A, 2002. 99(26): p. 16969-74.

154. Pitts, M.G., T.A. Combs, and S.E.F. D'Orazio, Neutrophils from Both Susceptible and Resistant Mice Efficiently Kill Opsonized Listeria monocytogenes. Infect Immun, 2018. 86(4).

155. Silver, K.L., et al., Complement driven innate immune response to malaria: fuelling severe malarial diseases. Cell Microbiol, 2010. 12(8): p. 1036-45.

156. Aoshi, T., et al., The cellular niche of Listeria monocytogenes infection changes rapidly in the spleen. Eur J Immunol, 2009. 39(2): p. 417-25.

157. Franken, L., et al., Splenic red pulp macrophages are intrinsically superparamagnetic and contaminate magnetic cell isolates. Sci Rep, 2015. 5: p. 12940.

158. Nagelkerke, S.Q., et al., Red pulp macrophages in the human spleen are a distinct cell population with a unique expression of Fc-gamma receptors. Blood Adv, 2018. 2(8): p. 941-953.

159. Mitchell, A.J., et al., Technical advance: autofluorescence as a tool for myeloid cell analysis. J Leukoc Biol, 2010. 88(3): p. 597-603. 
160. Nielsen, H., A. Kharazmi, and T.G. Theander, Suppression of blood monocyte and neutrophil chemotaxis in acute human malaria. Parasite Immunol, 1986. 8(6): p. 541-50.

161. Dasari, P., et al., Digestive vacuole of Plasmodium falciparum released during erythrocyte rupture dually activates complement and coagulation. Blood, 2012. 119(18): p. 4301-10.

162. Dasari, P., et al., Digestive vacuoles of Plasmodium falciparum are selectively phagocytosed by and impair killing function of polymorphonuclear leukocytes. Blood, 2011. 118(18): p. 4946-56.

163. Schwarzer, E., et al., Phagocytosis of the malarial pigment, hemozoin, impairs expression of major histocompatibility complex class II antigen, CD54, and CD11c in human monocytes. Infect Immun, 1998. 66(4): p. 1601-6.

164. Schwarzer, E. and P. Arese, Phagocytosis of malarial pigment hemozoin inhibits NADPH-oxidase activity in human monocyte-derived macrophages. Biochim Biophys Acta, 1996. 1316(3): p. 169-75.

165. Kim, C.C., et al., Splenic red pulp macrophages produce type I interferons as early sentinels of malaria infection but are dispensable for control. PLoS One, 2012. 7(10): p. e48126.

166. Domon, H., et al., Neutrophil Elastase Subverts the Immune Response by Cleaving Toll-Like Receptors and Cytokines in Pneumococcal Pneumonia. Front Immunol, 2018. 9: p. 732.

167. Champagne, B., et al., Proteolytic cleavage of ICAM-1 by human neutrophil elastase. J Immunol, 1998. 161(11): p. 6398-405.

168. Takem, E.N., A. Roca, and A. Cunnington, The association between malaria and non-typhoid Salmonella bacteraemia in children in sub-Saharan Africa: a literature review. Malar J, 2014. 13: p. 400.

169. Mtove, G., et al., Invasive salmonellosis among children admitted to a rural Tanzanian hospital and a comparison with previous studies. PLoS One, 2010. 5(2): p. e9244.

170. Hoffmann, O., et al., Interplay of pneumococcal hydrogen peroxide and hostderived nitric oxide. Infect Immun, 2006. 74(9): p. 5058-66.

171. Canvin, J.R., et al., The role of pneumolysin and autolysin in the pathology of pneumonia and septicemia in mice infected with a type 2 pneumococcus. J Infect Dis, 1995. 172(1): p. 119-23.

172. Standish, A.J. and J.N. Weiser, Human neutrophils kill Streptococcus pneumoniae via serine proteases. J Immunol, 2009. 183(4): p. 2602-9.

173. Schwarzer, E., et al., Impairment of macrophage functions after ingestion of Plasmodium falciparum-infected erythrocytes or isolated malarial pigment. J Exp Med, 1992. 176(4): p. 1033-41.

174. Schwarzer, E., et al., Phagocytosis of malarial pigment haemozoin by human monocytes: a confocal microscopy study. Parasitology, 2001. 123(Pt 2): p. 12531.

175. Witter, A.R., B.M. Okunnu, and R.E. Berg, The Essential Role of Neutrophils during Infection with the Intracellular Bacterial Pathogen Listeria monocytogenes. J Immunol, 2016. 197(5): p. 1557-65. 
176. Egan, T.J., Haemozoin formation. Mol Biochem Parasitol, 2008. 157(2): p. 12736.

177. Van den Steen, P.E., et al., Immunopathology and dexamethasone therapy in a new model for malaria-associated acute respiratory distress syndrome. Am J Respir Crit Care Med, 2010. 181(9): p. 957-68.

178. Eugenin, E.A., J.A. Martiney, and J.W. Berman, The malaria toxin hemozoin induces apoptosis in human neurons and astrocytes: Potential role in the pathogenesis of cerebral malaria. Brain Res, 2019. 1720: p. 146317.

179. Parroche, P., et al., Malaria hemozoin is immunologically inert but radically enhances innate responses by presenting malaria DNA to Toll-like receptor 9. Proc Natl Acad Sci U S A, 2007. 104(6): p. 1919-24.

180. Schwarzer, E., et al., Phagocytosis of P. falciparum malarial pigment hemozoin by human monocytes inactivates monocyte protein kinase $C$. Biochim Biophys Acta, 1993. 1181(1): p. 51-4.

181. Sherry, B.A., et al., Malaria-specific metabolite hemozoin mediates the release of several potent endogenous pyrogens (TNF, MIP-1 alpha, and MIP-1 beta) in vitro, and altered thermoregulation in vivo. J Inflamm, 1995. 45(2): p. 85-96.

182. Giribaldi, G., et al., Involvement of inflammatory chemokines in survival of human monocytes fed with malarial pigment. Infect Immun, 2010. 78(11): p. 4912-21.

183. Jani, D., et al., HDP-a novel heme detoxification protein from the malaria parasite. PLoS Pathog, 2008. 4(4): p. e1000053.

184. Lin, J.W., et al., Replication of Plasmodium in reticulocytes can occur without hemozoin formation, resulting in chloroquine resistance. J Exp Med, 2015. 212(6): p. 893-903.

185. Carney, C.K., et al., The basis of the immunomodulatory activity of malaria pigment (hemozoin). J Biol Inorg Chem, 2006. 11(7): p. 917-29.

186. Schwarzer, E., et al., Malaria-parasitized erythrocytes and hemozoin nonenzymatically generate large amounts of hydroxy fatty acids that inhibit monocyte functions. Blood, 2003. 101(2): p. 722-8.

187. Ercoli, G., et al., Intracellular replication of Streptococcus pneumoniae inside splenic macrophages serves as a reservoir for septicaemia. Nat Microbiol, 2018. 3(5): p. 600-610.

188. Long, T.T., et al., Influence of antimalarial treatment on acquisition of immunity in Plasmodium berghei NK65 malaria. Clin Diagn Lab Immunol, 2002. 9(4): p. 933-4.

189. Fu, Y., et al., Comparative histopathology of mice infected with the 17XL and 17XNL strains of Plasmodium yoelii. J Parasitol, 2012. 98(2): p. 310-5.

190. Kaplan, E.L., T. Laxdal, and P.G. Quie, Studies of polymorphonuclear leukocytes from patients with chronic granulomatous disease of childhood: bactericidal capacity for streptococci. Pediatrics, 1968. 41(3): p. 591-9.

191. Barbuti, G., et al., Streptococcus pneumoniae modulates the respiratory burst response in human neutrophils. FEMS Immunol Med Microbiol, 2010. 60(1): p. 57-62.

192. Segal, A.W., How neutrophils kill microbes. Annu Rev Immunol, 2005. 23: p. 197-223. 
193. Uriarte, S.M., et al., Granule exocytosis contributes to priming and activation of the human neutrophil respiratory burst. J Immunol, 2011. 187(1): p. 391-400.

194. Miralda, I., et al., Human Neutrophil Granule Exocytosis in Response to Mycobacterium smegmatis. Pathogens, 2020. 9(2).

195. Schneider, C., et al., IVIG regulates the survival of human but not mouse neutrophils. Sci Rep, 2017. 7(1): p. 1296.

196. Sun, K., et al., Interleukin-12 promotes gamma interferon-dependent neutrophil recruitment in the lung and improves protection against respiratory Streptococcus pneumoniae infection. Infect Immun, 2007. 75(3): p. 1196-202.

197. Marriott, H.M., et al., Contrasting roles for reactive oxygen species and nitric oxide in the innate response to pulmonary infection with Streptococcus pneumoniae. Vaccine, 2007. 25(13): p. 2485-90.

198. Vethanayagam, R.R., et al., Role of NADPH oxidase versus neutrophil proteases in antimicrobial host defense. PLoS One, 2011. 6(12): p. e28149.

199. Reeves, E.P., et al., Killing activity of neutrophils is mediated through activation of proteases by K+ flux. Nature, 2002. 416(6878): p. 291-7.

200. Sercundes, M.K., et al., Targeting Neutrophils to Prevent Malaria-Associated Acute Lung Injury/Acute Respiratory Distress Syndrome in Mice. PLoS Pathog, 2016. 12(12): p. e1006054.

201. Bandura, D.R., et al., Mass cytometry: technique for real time single cell multitarget immunoassay based on inductively coupled plasma time-of-flight mass spectrometry. Anal Chem, 2009. 81(16): p. 6813-22.

202. Bjornson, Z.B., G.P. Nolan, and W.J. Fantl, Single-cell mass cytometry for analysis of immune system functional states. Curr Opin Immunol, 2013. 25(4): p. 484-94.

203. Facer, C.A., Direct Coombs antiglobulin reactions in Gambian children with Plasmodium falciparum malaria. II. Specificity of erythrocyte-bound IgG. Clin Exp Immunol, 1980. 39(2): p. 279-88.

204. Facer, C.A., R.S. Bray, and J. Brown, Direct Coombs antiglobulin reactions in Gambian children with Plasmodium falciparum malaria. I. Incidence and class specificity. Clin Exp Immunol, 1979. 35(1): p. 119-27.

205. Warren, J., et al., Increased susceptibility of C1q-deficient mice to Salmonella enterica serovar Typhimurium infection. Infect Immun, 2002. 70(2): p. 551-7.

206. Hyams, C., et al., The Streptococcus pneumoniae capsule inhibits complement activity and neutrophil phagocytosis by multiple mechanisms. Infect Immun, 2010. 78(2): p. 704-15.

207. Frita, R., et al., Simple flow cytometric detection of haemozoin containing leukocytes and erythrocytes for research on diagnosis, immunology and drug sensitivity testing. Malar J, 2011. 10: p. 74. 


\section{APPENDICES}

Terms, abbreviations, and definitions

Sp-Streptococcus pneumoniae aka Pneumococcus

Lm - Listeria monocytogenes

NTS - Non Typhoid Salmonella

Py - Plasmodium yoelii - rodent malaria

Pf - Plasmodium falciparum - causes most cases of human malaria

$\mathbf{H z}$ - hemozoin - Plasmodium parasite biocrystal byproduct

Sepsis - bacterial infection in the blood

Bacteremia - bacterial infection in the blood

Hemolysis - lysis (bursting open) of red blood cells, like when parasites burst out. Kills the cell

Parasitemia - \% of red blood cells that are infected with the parasite

hyperparasitemia - high levels of parasitized red blood cells (over 5\% in humans, over $20 \%$ in mice)

Colony forming units (CFU) - the unit that we use to measure amount of bacteria (1 $\mathrm{CFU}=\sim 1$ single bacterium)

Neutrophil/polymorphonuclear cells (PMN) - fast acting innate immune cells. they enter an infected tissue and gobble up bacteria and kill them quickly

Macrophages - more long term/always ready innate immune cells. Also gobble up and kill bacteria, but not as quickly.

Flow cytometry - method for counting and characterizing cells. Uses lasers to detect different things and can tell you what type of immune cell are in a sample, or how the cells are functioning

HO-1 - Heme oxygenase-1: an enzyme produced after hemolysis, weakens some immune cell functions

Sn PP - a molecule that inhibits HO-1 activity

IL-10 - an anti-inflammatory signal made by cells when the immune system is trying to regulate or decrease potentially harmful levels of inflammation.

Anti-IL-10 antibody - an antibody treatment that blocks the activity of IL-10

Reactive oxygen species (ROS) - chemicals like hydrogen peroxide, free oxygen $\left(\mathrm{O}_{2}\right)$ produced by innate immune cells to kill bacteria

Opsonization - Molecules within the blood (serum) that can attach to pathogens and label them for destruction. Immune cells can find opsonized bacteria easier and have a more robust response against them

Serum - everything in the blood other than red blood cells(or other cells) and coagulation factors.

PMA - A chemical that induces ROS production from immune cells when put into culture

DCF - A chemical that fluoresces in the presence of ROS inside of cells. Used to measure the amount of ROS a cell is producing. 


\section{LAY AUDIENCE SUMMARY}

My project is focused on understanding how malaria weakens immunity to bacterial infections. I identified a parasite by-product that is capable of causing this immune suppression. I also created and characterized the first model system of a Plasmodium and Pneumococcus coinfection, a common and clinically important coinfection in sub-Saharan Africa, and demonstrated that the Plasmodium infection enhances the severity and mortality of pneumococcus. Finally, I identified immune cell functions that are defective following malaria, one of which is caused directly by a parasite byproduct. 


\title{
CURRICULUM VITAE
}

\author{
Christopher L. Harding \\ Louisville, KY $40204 \cdot(360) 561-5204$ \\ clhard08@louisville.edu
}

I am an enthusiastic microbiologist with a strong background in innate immunology and murine models of bacterial infections. Currently, I am seeking a postdoctoral position in the field of bacterial pathogenesis with a focus on host-pathogen interactions and immune responses to infectious diseases.

\section{EDUCATION}

AUGUST 2020

DOCTOR OF PHILOSOPHY, UNIVERSITY OF LOUISVILLE

Microbiology \& Immunology

- Dissertation Title: Plasmodium-derived hemozoin impairs antibacterial innate

immunity to systemic infections. Defended June 26, 2020

- Laboratory of Nathan W. Schmidt, PhD

APRIL 2016

MASTER OF SCIENCE, UNIVERSITY OF LOUISVILLE

Microbiology \& Immunology

AUGUST 2011

BACHELOR OF ARTS AND SCIENCE, UNIVERSITY OF COLORADO

Ecology and Evolutionary Biology

\section{RESEARCH EXPERIENCE}

$2014-2020$

RESEARCH FELLOW, UNIVERSITY OF LOUISVILLE

Department of Microbiology and Immunology: Lab of Dr. Nathan W. Schmidt

- Studied how Plasmodium infection impairs innate immunity to bacterial coinfections in mouse models of Listeria monocytogenes, Streptococcus pneumoniae, and Salmonella Typhimurium infection.

- Performed analysis of changes in myeloid cell populations of the spleen over the course of Plasmodium infection with flow cytometry.

- Examined antimicrobial functions of primary innate immune cells isolated from mouse spleen or bone marrow. 
2012 - 2014

\section{RESEARCH SCIENTIST II, UNIVERSITY OF WASHINGTON}

Department of Microbiology \& Department of Internal Medicine: Lab of Dr. Pradeep K. Singh

- Studied the in vivo virulence, persistence and antimicrobial resistance of Pseudomonas aeurginosa, Staphylococcus aureus, Acinetobacter baumanii, and Burkholderia thailandensis. Mouse models: Acute lung infection, chronic/acute wound infection, intraperitoneal infection

- Contributed to studies of bacterial persistence in colony biofilms.

- Assisted in collecting bacterial samples from explanted lungs of cystic fibrosis patients.

\section{$2012-2013$}

\section{RESEARCH SCIENTIST II, UNIVERSITY OF WASHINGTON}

Department of Dermatology: Lab of Dr. John E. Olerud

- Studied the role of bacterial biofilms in wound healing and subcutaneous implant models

- Prepared and analyzed histological tissue samples for immunohistochemistry and provided bacterial analysis for wound and implant studies.

$2009-2011$

UNDERGRADUATE RESEARCH ASSISTANT, UNIVERSITY OF COLORADO

Department of Neuroscience: Lab of Dr. Linda R. Watkins

- Performed cranial meninges surgery on rats. Handled and habituated rats. Prepared rat brain tissue for analysis with paraformaldehyde perfusions.

- Analyzed rat brain tissue images via densitometry to quantify glial cell activation in response to chronic migraine pain.

\section{PUBLICATIONS}

Harding, C.L., Villarino N, Valente E, Schwarzer E, and Schmidt N (2020). Plasmodium impairs antibacterial innate immunity to systemic infections in part through hemozoin-bound bioactive molecules. Front. Cell. Infect. Microbiol. doi: 10.3389/fcimb.2020.00328.

Morgan, S. J., Lippman, S. I., Bautista, G. E., Harrison, J. J., Harding, C. L., Gallagher, L. A., ... Singh, P. K. (2019). Bacterial fitness in chronic wounds appears to be mediated by the capacity for high-density growth, not virulence or biofilm functions. PLoS pathogens, 15(3), e1007511. doi:10.1371/journal.ppat.1007511

Villarino, N. F., LeCleir, G. R., Denny, J. E., Dearth, S. P., Harding, C. L., Sloan, S. S., ... Schmidt, N. W. (2016). Composition of the gut microbiota modulates the 
severity of malaria. Proceedings of the National Academy of Sciences of the

United States of America, 113(8), 2235-2240. doi:10.1073/pnas.1504887113

Schweppe, D. K., Harding, C. L, Chavez, J. D., Wu, X., Ramage, E., Singh, P. K., ... Bruce, J. E. (2015). Host-Microbe Protein Interactions during Bacterial Infection. Chemistry \& biology, 22(11), 1521-1530.

doi:10.1016/j.chembiol.2015.09.015

Jorth, P., Staudinger, B. J., Wu, X., Hisert, K. B., Hayden, H., Garudathri, J., Harding C. L. ... Singh, P. K. (2015). Regional Isolation Drives Bacterial Diversification within Cystic Fibrosis Lungs. Cell host \& microbe, 18(3), 307-319.

doi:10.1016/j.chom.2015.07.006

\section{PRESENTATIONS (FIRST AUTHOR)}

2019 American Society of Microbiology Biothreats Conference, Peer-toPeer Workshop Oral Presentation: "Plasmodium-Derived Hemozoin Impairs Innate Immunity to Bacterial Coinfections"

2019 Poster presenter at American Society of Microbiology Biothreats Conference "Plasmodium-Derived Hemozoin Impairs Innate Immunity to Bacterial Coinfections"

2018 University of Louisville Department of Microbiology and Immunology Seminar Series Oral Presentation: "Plasmodium Parasite Factor Impairs Immunity to Bacterial Coinfections"

2018 Poster presenter at Keystone Symposium: Myeloid Cells Conference: "Plasmodium Infection Increases Host Susceptibility to Systemic Bacterial Infection By modulating Innate Immune Response"

2017 Poster presenter at Research! Louisville: "Plasmodium Infection Increases Host Susceptibility to Systemic Bacterial Infection by Modulating Innate Immune Response"

2016 University of Louisville Department of Microbiology and Immunology Seminar Series Oral Presentation: "Exploring Malaria's Modulation of the Innate Immune Response to Bacterial Coinfection"

2016 Poster presenter at Research! Louisville: "Plasmodium infection increases host susceptibility to systemic bacterial infection by modulating innate immune response" 


\section{POSITIONS \& HONORS}

\section{LEADERSHIP POSITIONS:}

2017-2019 Outreach Coordinator for University of Louisville Science Policy and Outreach Group(SPOG)

2018-2019 Executive Board Member for Louisville Regional Science Fair (LRSF)

2017-2018 Student Representative for Department of Microbiology and Immunology Admissions Committee

2017-2018 Program Coordinator for Louisville Science Pathways Summer research internship program for local high school students

2015-2017 Graduate Student Council Representative for the Microbiology and Immunology Student Organization, Co-Chair of Health Science Campus Group

TEACHING \& MENTORING EXPERIENCE:

Mentored local high school student recipient of the Microbiology \& Immunology Summer Translational Experience Award.

$2018 \quad$ Attended Instructional Strategies in Science Education Course

2017-2018 Presented lecture titled "Immune Cell Functional Assays" in Methods in Microbiology and Immunology

2016 Completed STEM Graduate Teaching Academy

\section{SERVICE \& VOLUNTEERING:}

Volunteer for "Girls Rule! STEM Education Summit" for University of Louisville Science Policy and Outreach Group(SPOG)

2015-2019

2015-2016

2011

2010-2011
Science Fair Judge for the Louisville Regional Science Fair

Science Outreach Volunteer for GEAR UP Pre-Professional Academy Salmon Steward for Thurston County Parks and Recreation Science Fair Judge for Roche Colorado Regional Science Fair 